\title{
Reduced complexity model intercomparison project phase 1: Protocol, results and initial observations: supplementary information
}

Zebedee R. J. Nicholls ${ }^{1,2}$, Malte Meinshausen ${ }^{1,2,3}$, Jared Lewis ${ }^{1}$, Robert Gieseke ${ }^{3}$, Dietmar Dommenget ${ }^{4}$, Kalyn Dorheim ${ }^{5}$, Chen-Shuo Fan ${ }^{4}$, Jan S. Fuglestvedt ${ }^{6}$, Thomas Gasser ${ }^{7}$, Ulrich Golüke ${ }^{8}$, Philip Goodwin ${ }^{9}$, Elmar Kriegler ${ }^{3}$, Nicholas J. Leach ${ }^{10}$, Davide Marchegiani ${ }^{4}$, Yann Quilcaille ${ }^{7}$, Bjørn H. Samset ${ }^{6}$, Marit Sandstad ${ }^{6}$, Alexey N. Shiklomanov ${ }^{5}$, Ragnhild B. Skeie ${ }^{6}$, Christopher J. Smith ${ }^{11}$, Katsumasa Tanaka ${ }^{12,13}$, Junichi Tsutsui ${ }^{14}$, and Zhiang Xie ${ }^{4}$

\footnotetext{
${ }^{1}$ Australian-German Climate and Energy College, The University of Melbourne, Parkville, Victoria, Australia

${ }^{2}$ School of Earth Sciences, The University of Melbourne, Parkville, Victoria, Australia

${ }^{3}$ Potsdam Institute for Climate Impact Research (PIK), Member of the Leibniz Association, Potsdam, Germany

${ }^{4}$ Monash University, School of Earth, Atmosphere and Environment, Clayton, Victoria 3800, Australia

${ }^{5}$ Joint Global Change Research Institute, Pacific Northwest National Laboratory, College Park, MD, USA

${ }^{6}$ CICERO Center for International Climate Research, Oslo, Norway

${ }^{7}$ International Institute for Applied Systems Analysis (IIASA), Laxenburg, Austria

${ }^{8}$ BI Norwegian Business School, Nydalsveien 37, 0484 Oslo, Norway

${ }^{9}$ School of Ocean and Earth Science, University of Southampton, Southampton, UK

${ }^{10}$ Department of Physics, Atmospheric Oceanic and Planetary Physics, University of Oxford, United Kingdom

${ }^{11}$ Priestley International Centre for Climate, University of Leeds, UK

${ }^{12}$ National Institute for Environmental Studies (NIES), Tsukuba, Japan

${ }^{13}$ Laboratoire des Sciences du Climat et de l'Environnement (LSCE), Commissariat à l'énergie atomique et aux énergies alternatives (CEA), Gif sur Yvette, France

${ }^{14}$ Central Research Institute of Electric Power Industry, Abiko, Japan

Correspondence: Zebedee Nicholls (zebedee.nicholls@ climate-energy-college.org)
} 


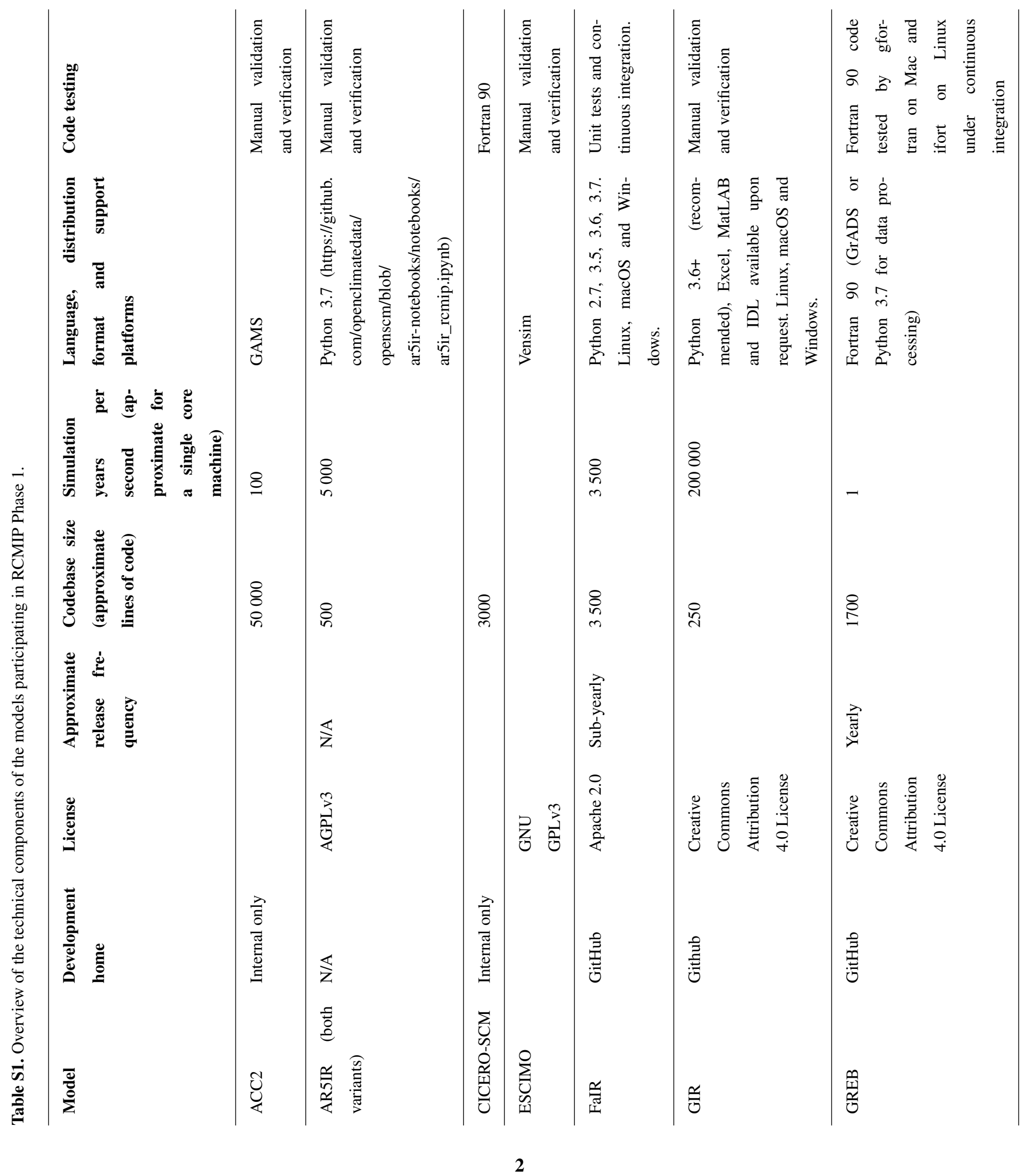




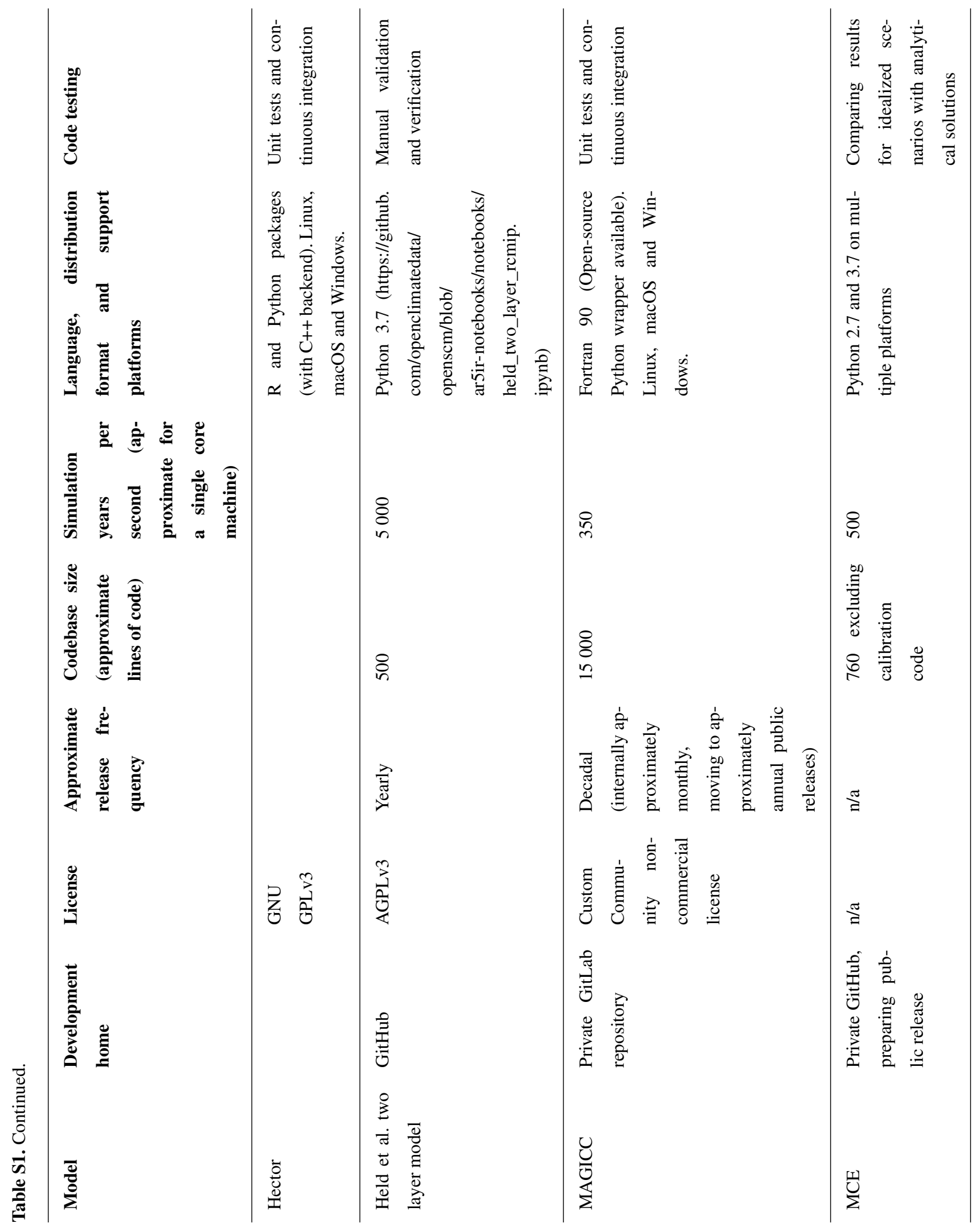




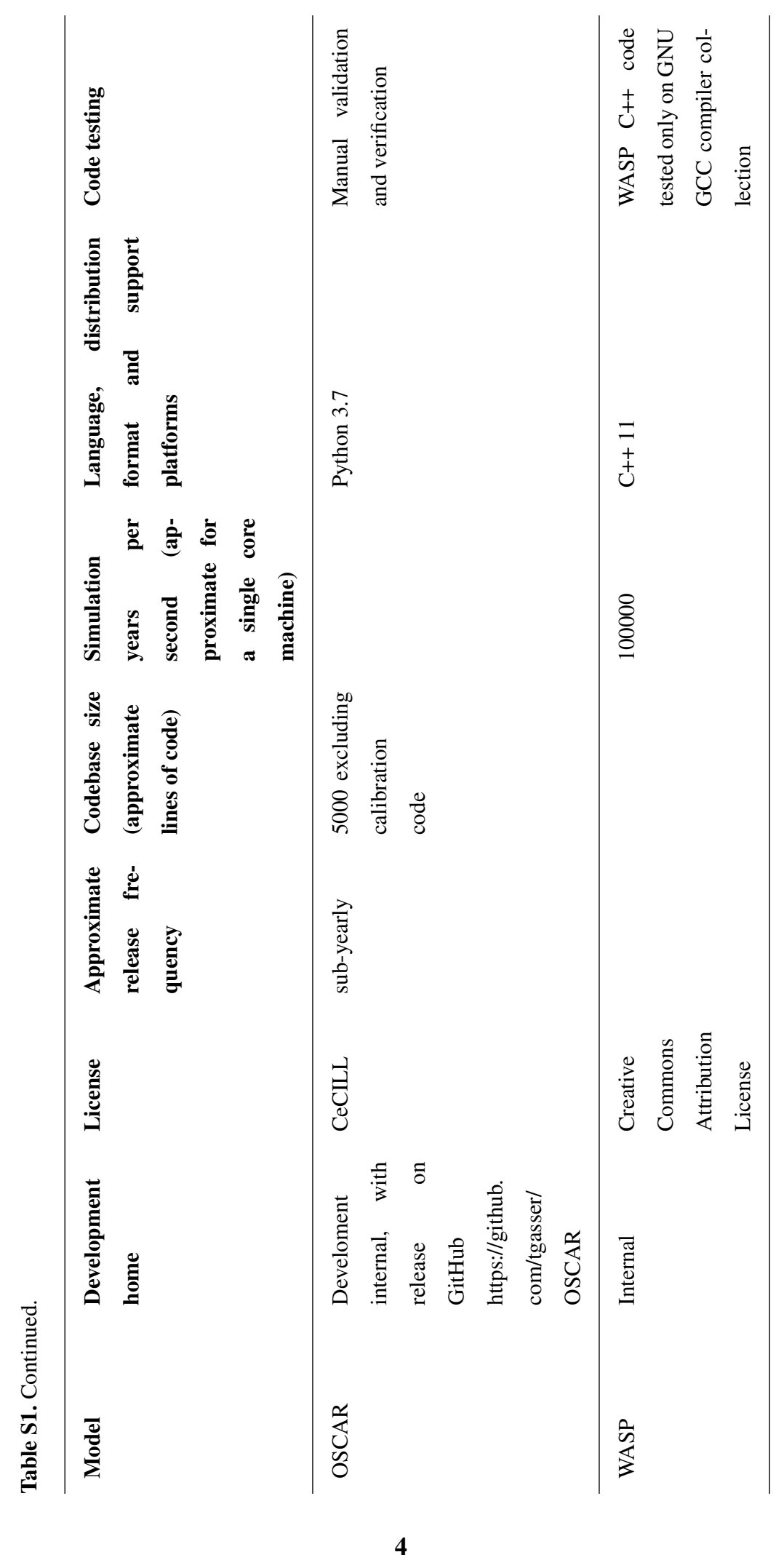



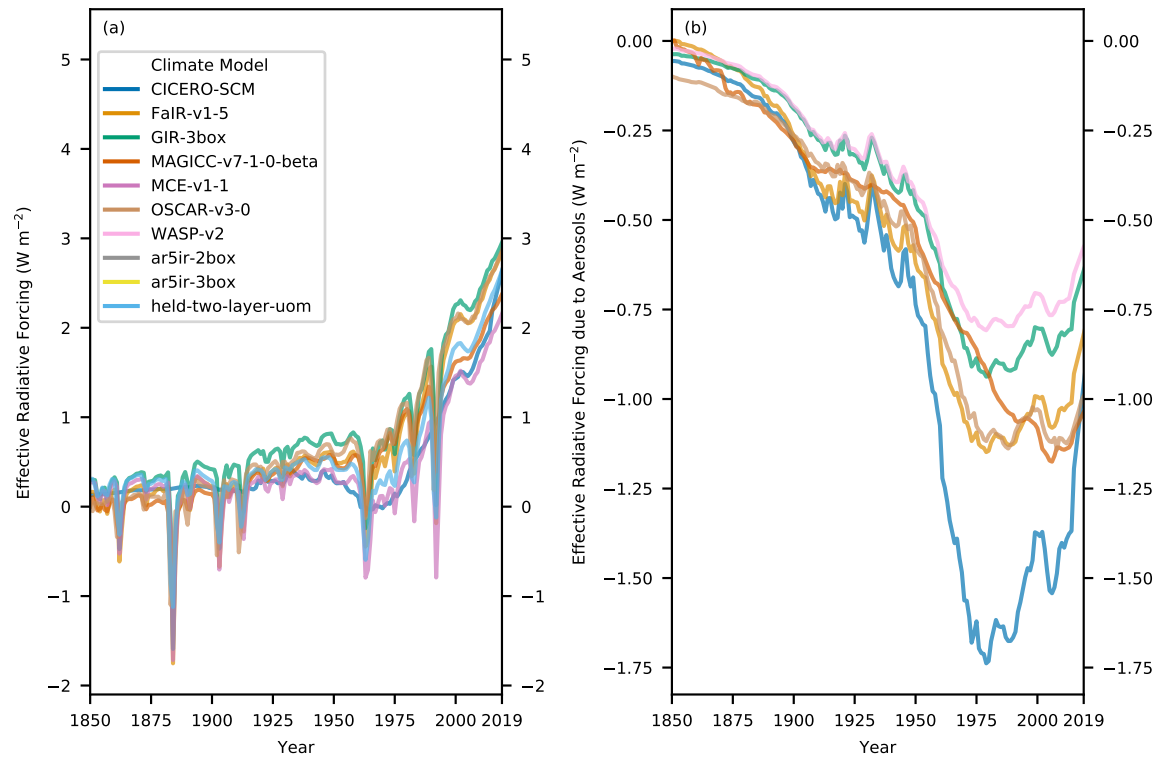

Figure S1. Historical effective radiative forcing for RCMIP models in illustrative configurations. In order to provide timeseries up until 2019 , we have used data from the combination of historical and ssp585 simulations. (a) - total effective radiative forcing; (b) - aerosol effective radiative forcing. 
Table S2. Emulation scores and equilbrium climate sensitivities (ECSs) for RCMIP model calibrations. In parentheses we show the number of simulations available for each model variant.

\begin{tabular}{|c|c|c|c|}
\hline & & $\operatorname{ECS}(\mathbf{K})$ & RMSE (K) \\
\hline Target CMIP6 model & RCMIP model & & \\
\hline AWI-CM-1-1-MR_r1i1p1f1 (5) & MAGICC-v7-1-0-beta (5) & 3.22 & 0.16 \\
\hline \multirow[t]{5}{*}{ BCC-CSM2-MR_r1i1p1f1 (6) } & MCE-v1-1 (2) & 2.90 & 0.21 \\
\hline & MAGICC-v7-1-0-beta (6) & 2.83 & 0.16 \\
\hline & ar5ir-2box (2) & 7.35 & 0.13 \\
\hline & $\operatorname{ar} 5$ ir-3box (2) & 7.78 & 0.13 \\
\hline & held-two-layer-uom (2) & 2.63 & 0.13 \\
\hline \multirow[t]{5}{*}{ BCC-ESM1_r1i1p1f1 (4) } & MCE-v1-1 (2) & 2.96 & 0.12 \\
\hline & MAGICC-v7-1-0-beta (3) & 3.13 & 0.13 \\
\hline & ar5ir-2box (2) & 15.30 & 0.18 \\
\hline & $\operatorname{ar} 5 \operatorname{ir}-3 b o x(2)$ & 8.06 & 0.15 \\
\hline & held-two-layer-uom (2) & 2.31 & 0.12 \\
\hline \multirow[t]{6}{*}{ CanESM5_r1i1p1f1 (10) } & MCE-v1-1 (2) & 5.08 & 0.13 \\
\hline & hector|62381e71 (4) & 4.79 & 0.42 \\
\hline & MAGICC-v7-1-0-beta (10) & 5.72 & 0.30 \\
\hline & ar5ir-2box (2) & 5.24 & 0.19 \\
\hline & $\operatorname{ar} 5 \mathrm{ir}-3 \mathrm{box}(2)$ & 11.82 & 0.21 \\
\hline & held-two-layer-uom (2) & 3.14 & 0.30 \\
\hline \multirow[t]{3}{*}{ CanESM5_r1i1p2f1 (7) } & MCE-v1-1 (2) & 5.08 & 0.13 \\
\hline & hectorl62381e71 (4) & 4.79 & 0.43 \\
\hline & MAGICC-v7-1-0-beta (7) & 5.64 & 0.27 \\
\hline \multirow[t]{2}{*}{ CanESM5_r10i1p1f1 (5) } & hectorl62381e71 (2) & 4.79 & 0.29 \\
\hline & MAGICC-v7-1-0-beta (5) & 6.01 & 0.18 \\
\hline \multirow[t]{6}{*}{ CESM2-WACCM_r1i1p1f1 (6) } & MCE-v1-1 (2) & 3.85 & 0.15 \\
\hline & hector|62381e71 (3) & 4.17 & 0.26 \\
\hline & MAGICC-v7-1-0-beta (6) & 4.26 & 0.21 \\
\hline & ar5ir-2box (2) & 4.64 & 0.45 \\
\hline & ar5ir-3box (2) & 13.42 & 0.21 \\
\hline & held-two-layer-uom (2) & 2.55 & 0.13 \\
\hline
\end{tabular}


Table S2. Continued.

\begin{tabular}{|c|c|c|c|}
\hline & & $\operatorname{ECS}(\mathrm{K})$ & RMSE (K) \\
\hline Target CMIP6 model & RCMIP model & & \\
\hline \multirow[t]{6}{*}{ CESM2_r1i1p1f1 (6) } & MCE-v1-1 (2) & 4.20 & 0.17 \\
\hline & hector|62381e71 (3) & 4.00 & 0.67 \\
\hline & MAGICC-v7-1-0-beta (6) & 5.32 & 0.27 \\
\hline & $\operatorname{ar} 5$ ir-2box (2) & 5.40 & 0.24 \\
\hline & $\operatorname{ar} 5 \operatorname{ir}-3 b o x(2)$ & 8.31 & 0.24 \\
\hline & held-two-layer-uom (2) & 3.63 & 0.20 \\
\hline \multirow[t]{6}{*}{ CNRM-CM6-1_r1i1p1f2 (8) } & MCE-v1-1 (4) & 4.06 & 0.24 \\
\hline & hectorl62381e71 (5) & 3.86 & 0.36 \\
\hline & MAGICC-v7-1-0-beta (8) & 4.08 & 0.18 \\
\hline & $\operatorname{ar} 5 \mathrm{ir}-2 \mathrm{box}(4)$ & 8.13 & 0.43 \\
\hline & $\operatorname{ar} 5 \operatorname{ir}-3 b o x(4)$ & 9.12 & 0.43 \\
\hline & held-two-layer-uom (4) & 2.91 & 0.16 \\
\hline \multirow[t]{6}{*}{ CNRM-ESM2-1_r1i1p1f2 (10) } & MCE-v1-1 (2) & 4.02 & 0.20 \\
\hline & hectorl62381e71 (4) & 3.51 & 0.25 \\
\hline & MAGICC-v7-1-0-beta (9) & 3.71 & 0.18 \\
\hline & $\operatorname{ar} 5 \mathrm{ir}-2 \mathrm{box}(2)$ & 8.22 & 0.27 \\
\hline & $\operatorname{ar} 5 \operatorname{ir}-3 b o x(2)$ & 12.18 & 0.27 \\
\hline & held-two-layer-uom (2) & 2.29 & 0.17 \\
\hline \multirow[t]{2}{*}{ E3SM-1-0_r1i1p1f1 (2) } & MCE-v1-1 (2) & 5.10 & 0.17 \\
\hline & MAGICC-v7-1-0-beta (2) & 5.69 & 0.22 \\
\hline \multirow[t]{5}{*}{ EC-Earth3-Veg_r1i1p1f1 (7) } & MCE-v1-1 (2) & 4.13 & 0.19 \\
\hline & MAGICC-v7-1-0-beta (7) & 4.47 & 0.25 \\
\hline & $\operatorname{ar} 5 \operatorname{ir}-2$ box $(2)$ & 15.91 & 0.27 \\
\hline & $\operatorname{ar} 5$ ir-3box (2) & 8.32 & 0.22 \\
\hline & held-two-layer-uom (2) & 3.50 & 0.19 \\
\hline
\end{tabular}


Table S2. Continued.

\begin{tabular}{|c|c|c|c|}
\hline & & $\operatorname{ECS}(\mathbf{K})$ & RMSE (K) \\
\hline Target CMIP6 model & RCMIP model & & \\
\hline FGOALS-g3_r1i1p1f1 (4) & MAGICC-v7-1-0-beta (4) & 2.77 & 0.15 \\
\hline \multirow[t]{5}{*}{ GISS-E2-1-G_r1i1p1f1 (4) } & MCE-v1-1 (4) & 2.69 & 0.16 \\
\hline & MAGICC-v7-1-0-beta (4) & 2.81 & 0.19 \\
\hline & ar5ir-2box (4) & 5.24 & 0.15 \\
\hline & ar5ir-3box (4) & 18.98 & 0.58 \\
\hline & held-two-layer-uom (4) & 2.50 & 0.15 \\
\hline \multirow[t]{5}{*}{ GISS-E2-1-H_r1i1p1f1 (3) } & MCE-v1-1 (3) & 3.07 & 0.15 \\
\hline & MAGICC-v7-1-0-beta (3) & 3.20 & 0.16 \\
\hline & ar5ir-2box (3) & 16.68 & 0.16 \\
\hline & $\operatorname{ar} 5 \mathrm{ir}-3 \mathrm{box}(3)$ & 8.05 & 0.15 \\
\hline & held-two-layer-uom (3) & 2.48 & 0.14 \\
\hline \multirow[t]{4}{*}{ GISS-E2-2-G_r1i1p1f1 (3) } & MAGICC-v7-1-0-beta (3) & 2.88 & 0.19 \\
\hline & $\operatorname{ar} 5 \mathrm{ir}-2 \mathrm{box}(3)$ & 3.70 & 0.16 \\
\hline & $\operatorname{ar} 5 \operatorname{ir}-3 b o x(3)$ & 18.86 & 0.66 \\
\hline & held-two-layer-uom (3) & 1.90 & 0.14 \\
\hline \multirow[t]{6}{*}{ IPSL-CM6A-LR_r1i1p1f1 (9) } & MCE-v1-1 (4) & 3.83 & 0.25 \\
\hline & hector|62381e71 (6) & 3.07 & 0.67 \\
\hline & MAGICC-v7-1-0-beta (9) & 4.53 & 0.25 \\
\hline & $\operatorname{ar} 5 \mathrm{ir}-2 \mathrm{box}(4)$ & 13.57 & 0.34 \\
\hline & $\operatorname{ar} 5 \mathrm{ir}-3 \mathrm{box}(4)$ & 5.71 & 0.26 \\
\hline & held-two-layer-uom (4) & 4.57 & 0.29 \\
\hline IPSL-CM6A-LR_r1i1p1f2 (2) & MAGICC-v7-1-0-beta (2) & 4.43 & 0.21 \\
\hline \multirow[t]{3}{*}{ IPSL-CM6A-LR_r10i1p1f1 (3) } & MCE-v1-1 (1) & 3.83 & 0.21 \\
\hline & hector|62381e71 (1) & 3.07 & 0.40 \\
\hline & MAGICC-v7-1-0-beta (3) & 3.77 & 0.32 \\
\hline MCM-UA-1-0_r1i1p1f2 (4) & MAGICC-v7-1-0-beta (4) & 3.45 & 0.16 \\
\hline
\end{tabular}


Table S2. Continued.

\begin{tabular}{|c|c|c|c|}
\hline & & $\operatorname{ECS}(\mathbf{K})$ & RMSE (K) \\
\hline Target CMIP6 model & RCMIP model & & \\
\hline \multirow[t]{2}{*}{ MIROC6_r1i1p1f1 (14) } & MCE-v1-1 (4) & 2.44 & 0.28 \\
\hline & MAGICC-v7-1-0-beta (12) & 2.20 & 0.19 \\
\hline \multirow[t]{4}{*}{ MPI-ESM1-2-HR_r1i1p1f1 (2) } & MAGICC-v7-1-0-beta (2) & 2.90 & 0.15 \\
\hline & ar5ir-2box (2) & 8.02 & 0.16 \\
\hline & ar5ir-3box (2) & 6.08 & 0.16 \\
\hline & held-two-layer-uom (2) & 2.17 & 0.12 \\
\hline \multirow[t]{4}{*}{ NorCPM1_r1i1p1f1 (2) } & MAGICC-v7-1-0-beta (2) & 2.73 & 0.29 \\
\hline & $\operatorname{ar} 5$ ir-2box (2) & 7.24 & 0.13 \\
\hline & $\operatorname{ar} 5 \operatorname{ir}-3$ box $(2)$ & 8.60 & 0.23 \\
\hline & held-two-layer-uom (2) & 4.15 & 0.18 \\
\hline \multirow[t]{4}{*}{ NorESM2-LM_r1i1p1f1 (3) } & MCE-v1-1 (2) & 2.19 & 0.32 \\
\hline & MAGICC-v7-1-0-beta (2) & 2.27 & 0.22 \\
\hline & $\operatorname{ar} 5$ ir-2box (2) & 13.37 & 0.19 \\
\hline & $\operatorname{ar} 5$ ir-3box (2) & 12.48 & 0.19 \\
\hline \multirow[t]{2}{*}{ SAM0-UNICON_r1i1p1f1 (2) } & MCE-v1-1 (2) & 3.80 & 0.15 \\
\hline & MAGICC-v7-1-0-beta (2) & 3.42 & 0.24 \\
\hline \multirow[t]{5}{*}{ UKESM1-0-LL_r1i1p1f2 (9) } & MCE-v1-1 (2) & 5.31 & 0.16 \\
\hline & MAGICC-v7-1-0-beta (9) & 6.05 & 0.30 \\
\hline & ar5ir-2box (2) & 16.92 & 0.26 \\
\hline & ar5ir-3box (2) & 7.22 & 0.19 \\
\hline & held-two-layer-uom (2) & 4.11 & 0.19 \\
\hline
\end{tabular}



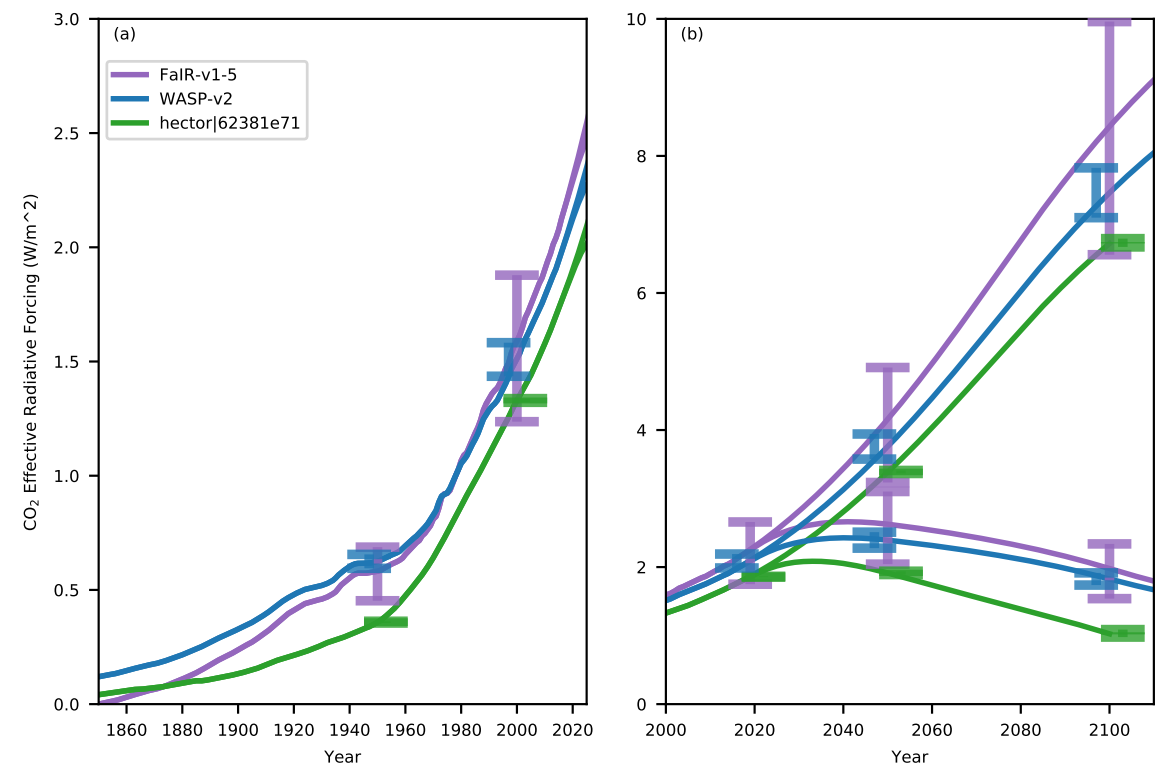

Figure S2. Probabilistic estimate of $\mathrm{CO}_{2}$ effective radiative forcing for ssp119 and ssp585 (note, for $\mathrm{Hector}_{\mathrm{CO}}$ radiative forcing is shown as effective radiative forcing is not available). (a) - historical period (1850-2025); (b) - projections (2000-2110). 

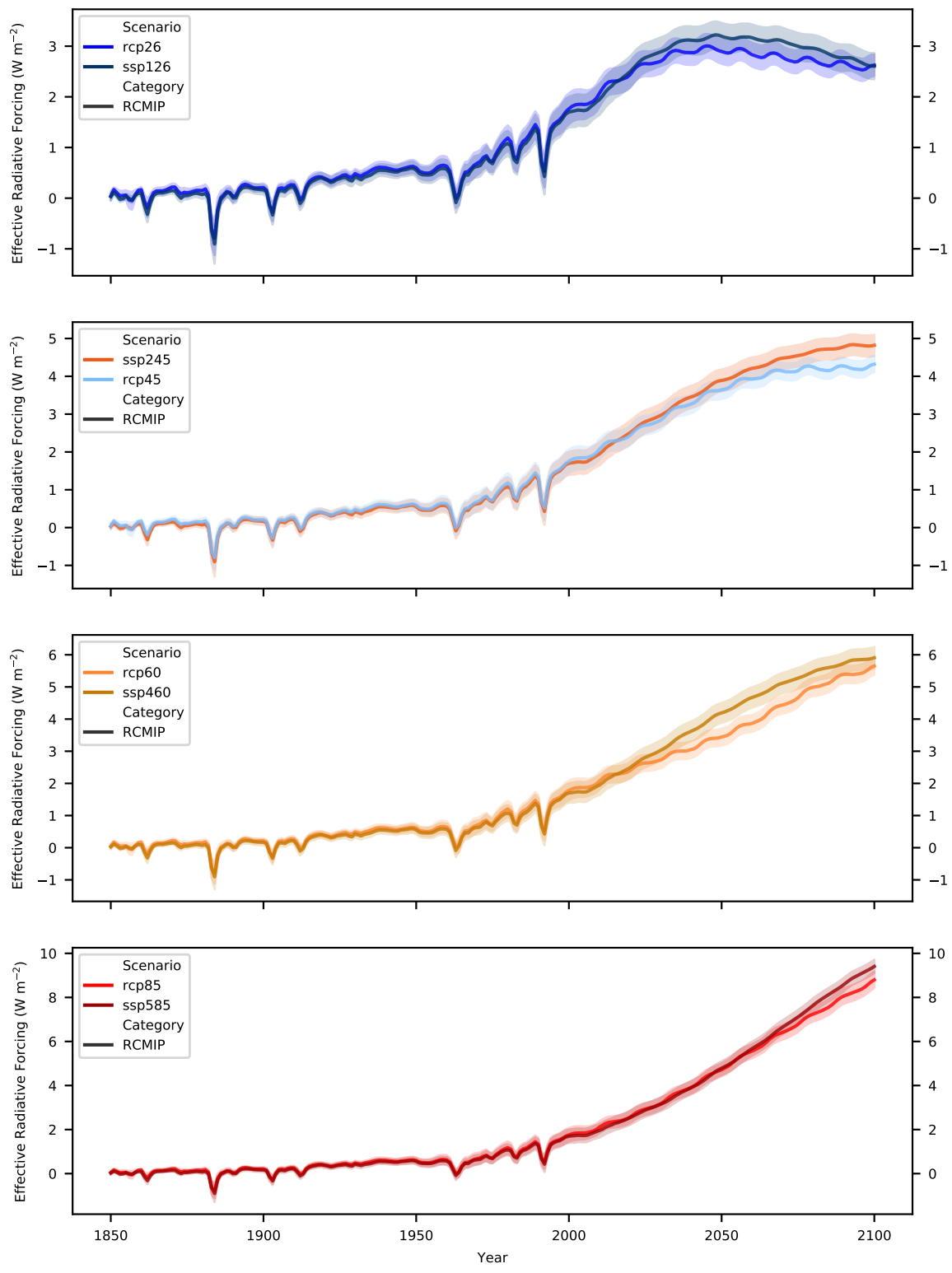

Figure S3. Comparison of effective radiative forcing projections under the RCPs and SSPs up until 2100. The coloured solid lines are RCMIP output where the RCP/SSP pair has been run with the same model in the same configuration. The plumes show the standard deviation of the available model results whilst the lines show the mean. 

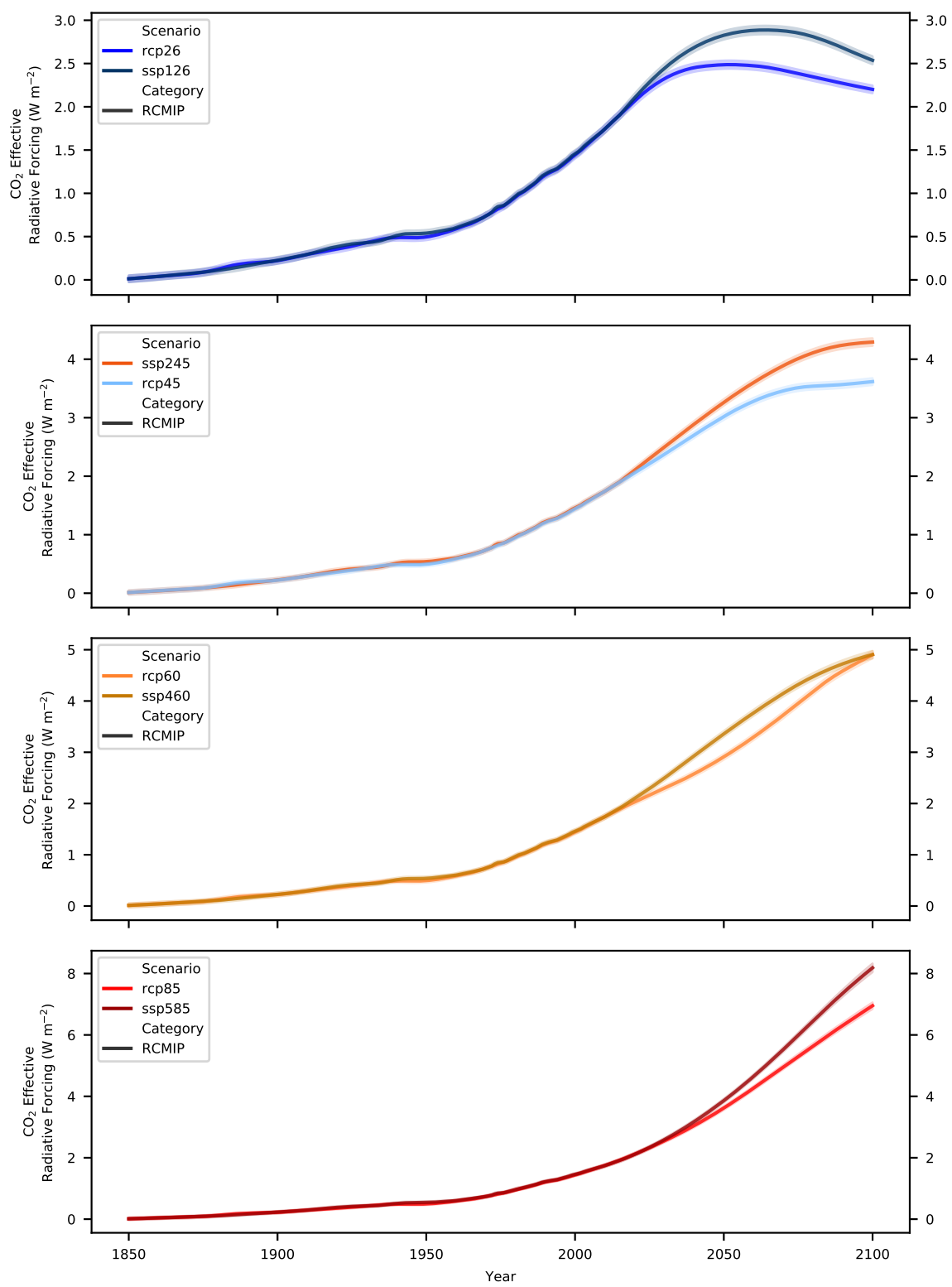

Figure S4. Comparison of $\mathrm{CO}_{2}$ effective radiative forcing projections under the RCPs and SSPs up until 2100 . The coloured solid lines are RCMIP output where the RCP/SSP pair has been run with the same model in the same configuration. The plumes show the standard deviation of the available model results whilst the lines show the mean. 

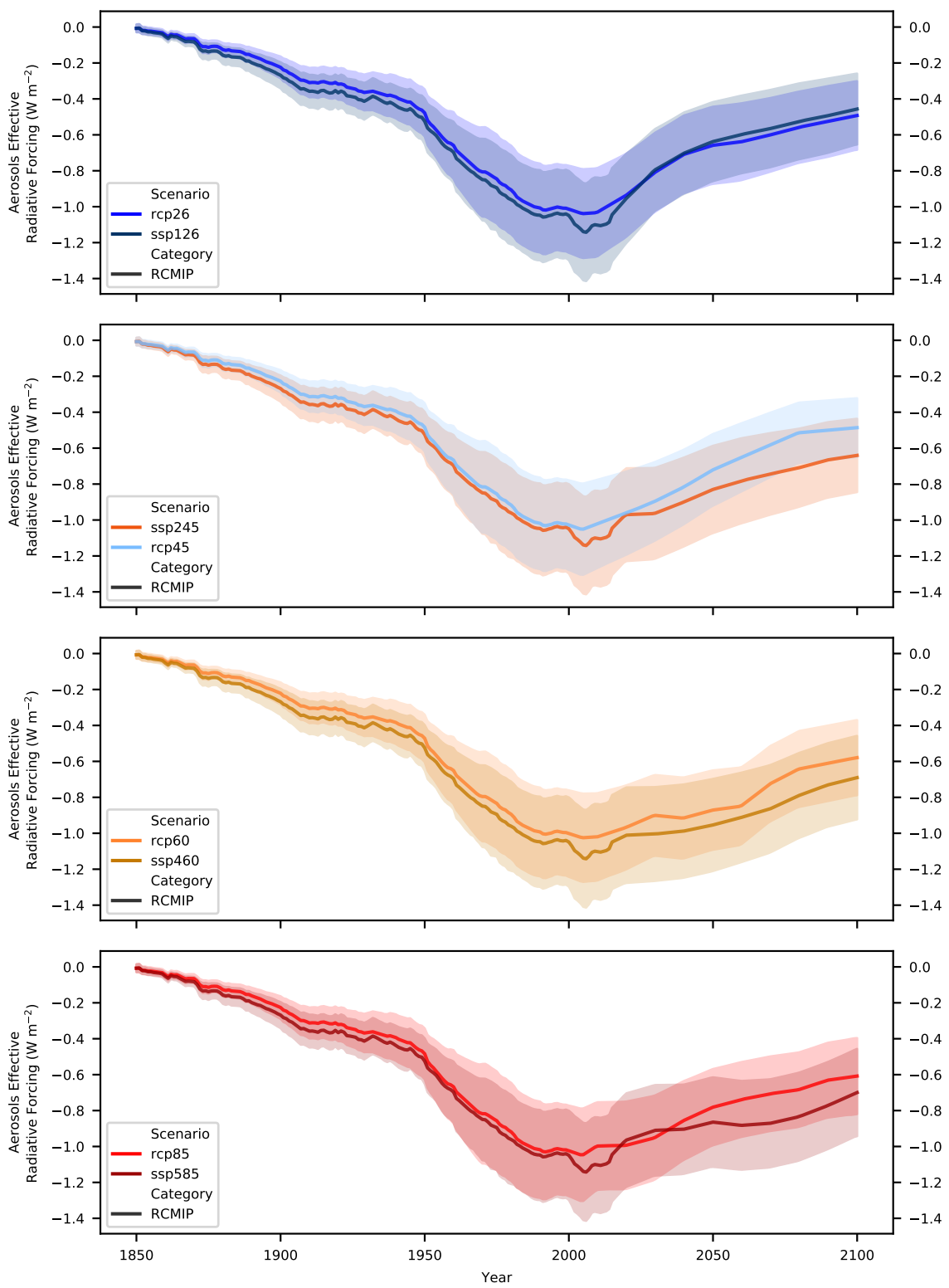

Figure S5. Comparison of aerosols effective radiative forcing projections under the RCPs and SSPs up until 2100 . The coloured solid lines are RCMIP output where the RCP/SSP pair has been run with the same model in the same configuration. The plumes show the standard deviation of the available model results whilst the lines show the mean. 

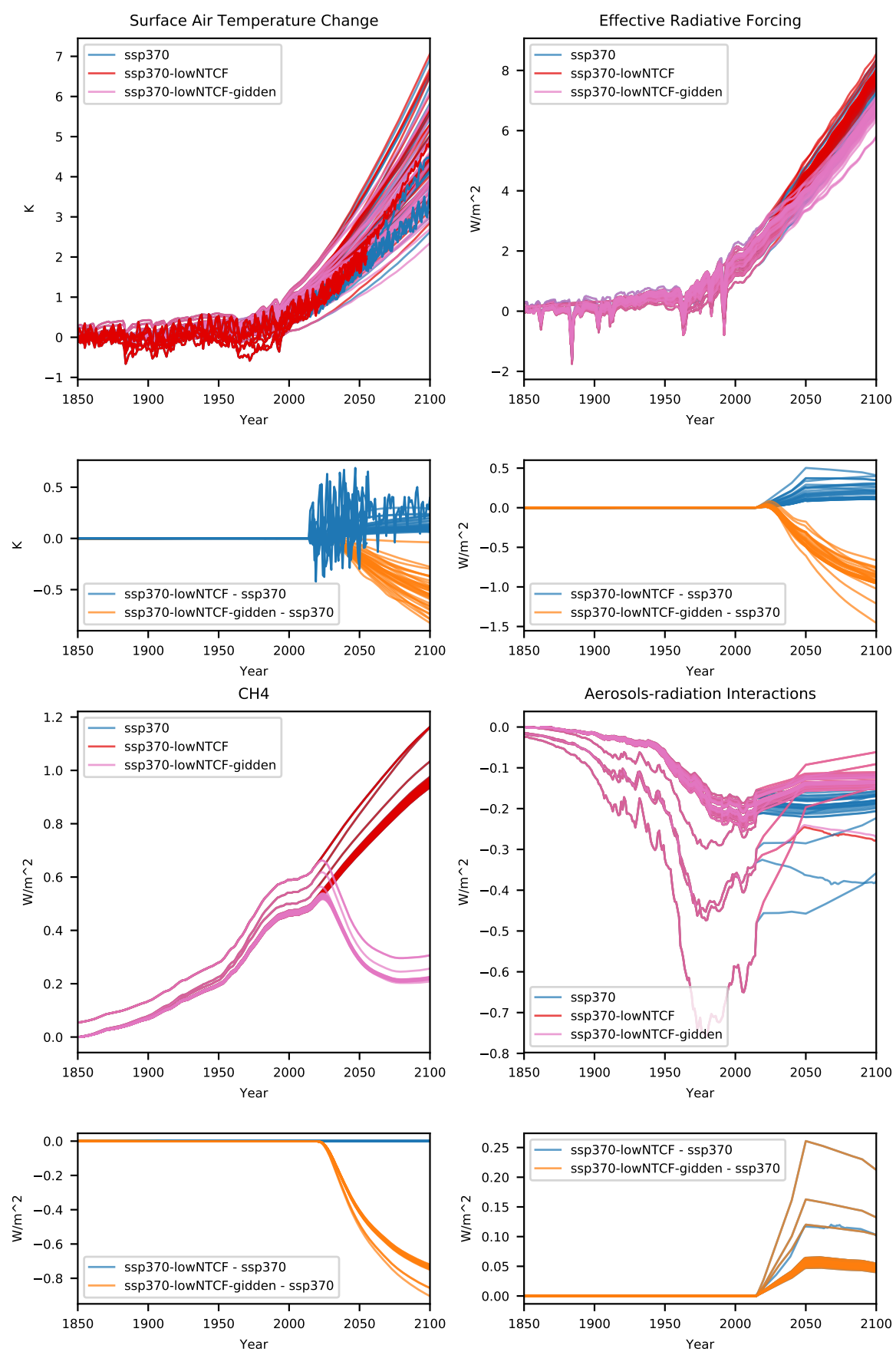

Figure S6. Response of RCMIP models to a reduction in near-term climate forcers. Results are from RCMIP models, except for temperature lines with natural variability which are CMIP6 results. The ssp370-lowNTCF scenario results in a small warming signal relative to ssp370, the magnitude of which varies by RCM. For comparison, we also include ssp370-lowNTCF as quantified by Gidden et al. (2019) (labelled 'ssp370-lowntcf-gidden'). This implementation also includes reductions in methane and so a strong cooling signal is seen instead. 

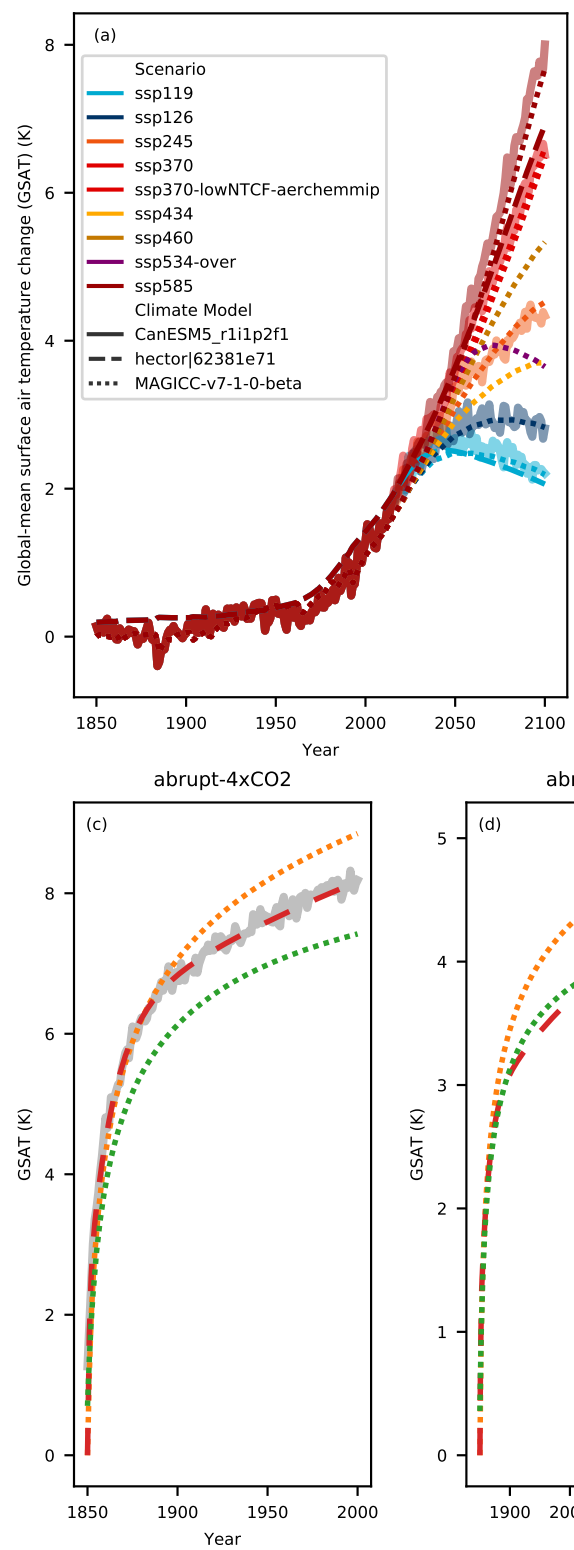

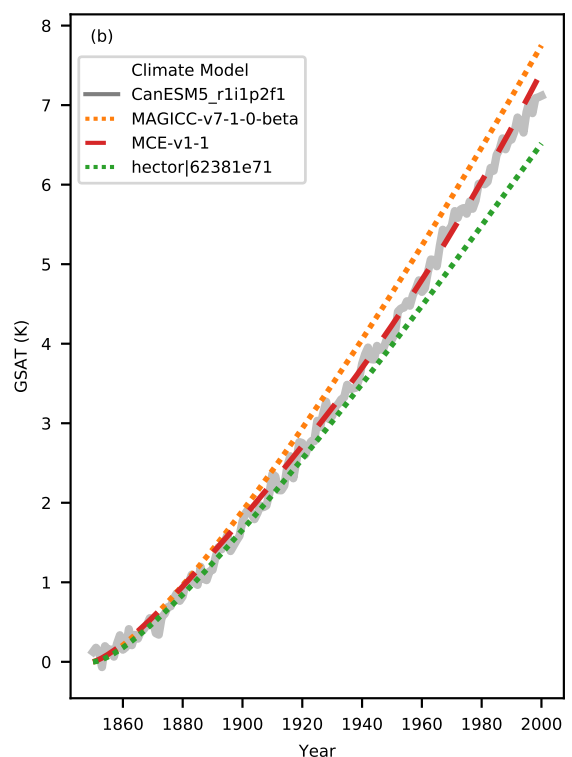

abrupt-0p $5 \times \mathrm{CO} 2$
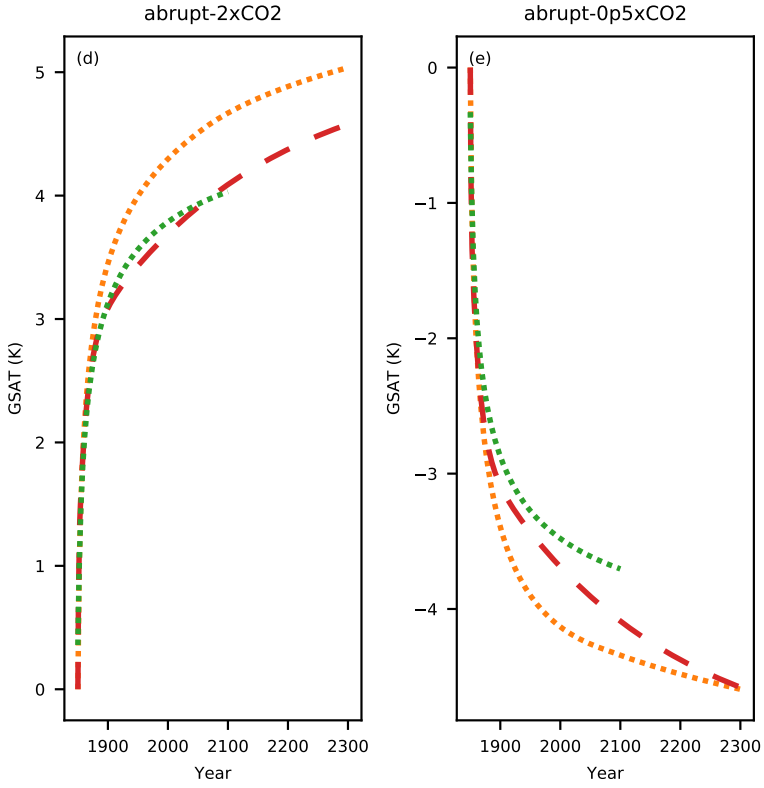

Figure S7. Emulation of CanESM5_r1i1p2f1 by RCMs in RCMIP Phase 1. The thick transparent lines are the target CMIP6 model output (here from CanESM5_r1i1p2f1). The thin lines are emulations from different RCMs. Panel (a) shows results for scenario based experiments while panels (b) - (e) show results for idealised CO2-only experiments (note that panels (b) - (e) share the same legend). 

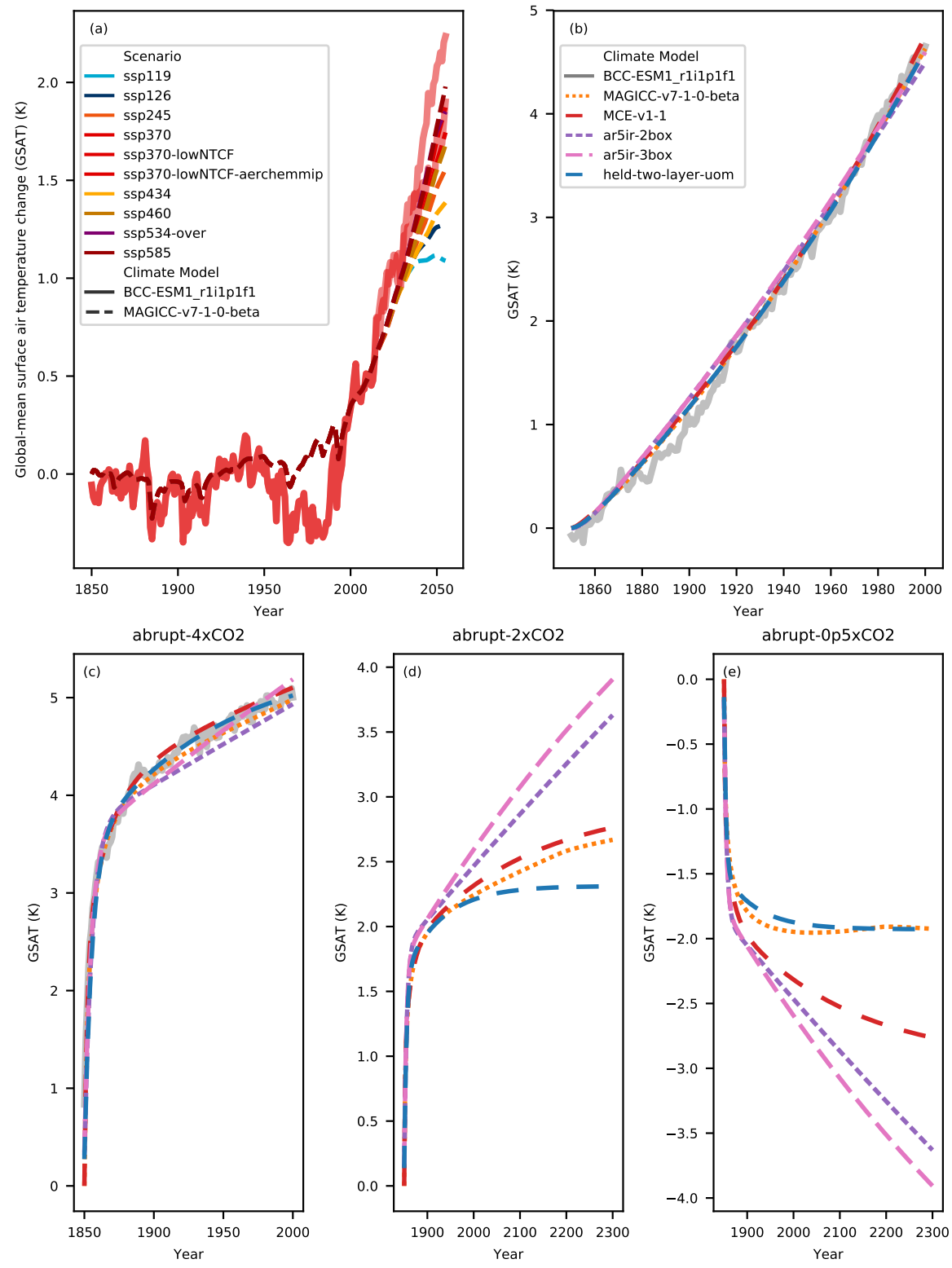

Figure S8. Emulation of BCC-ESM1_r1i1p1f1 by RCMs in RCMIP Phase 1. The thick transparent lines are the target CMIP6 model output (here from BCC-ESM1_r1i1p1f1). The thin lines are emulations from different RCMs. Panel (a) shows results for scenario based experiments while panels (b) - (e) show results for idealised CO2-only experiments (note that panels (b) - (e) share the same legend). 

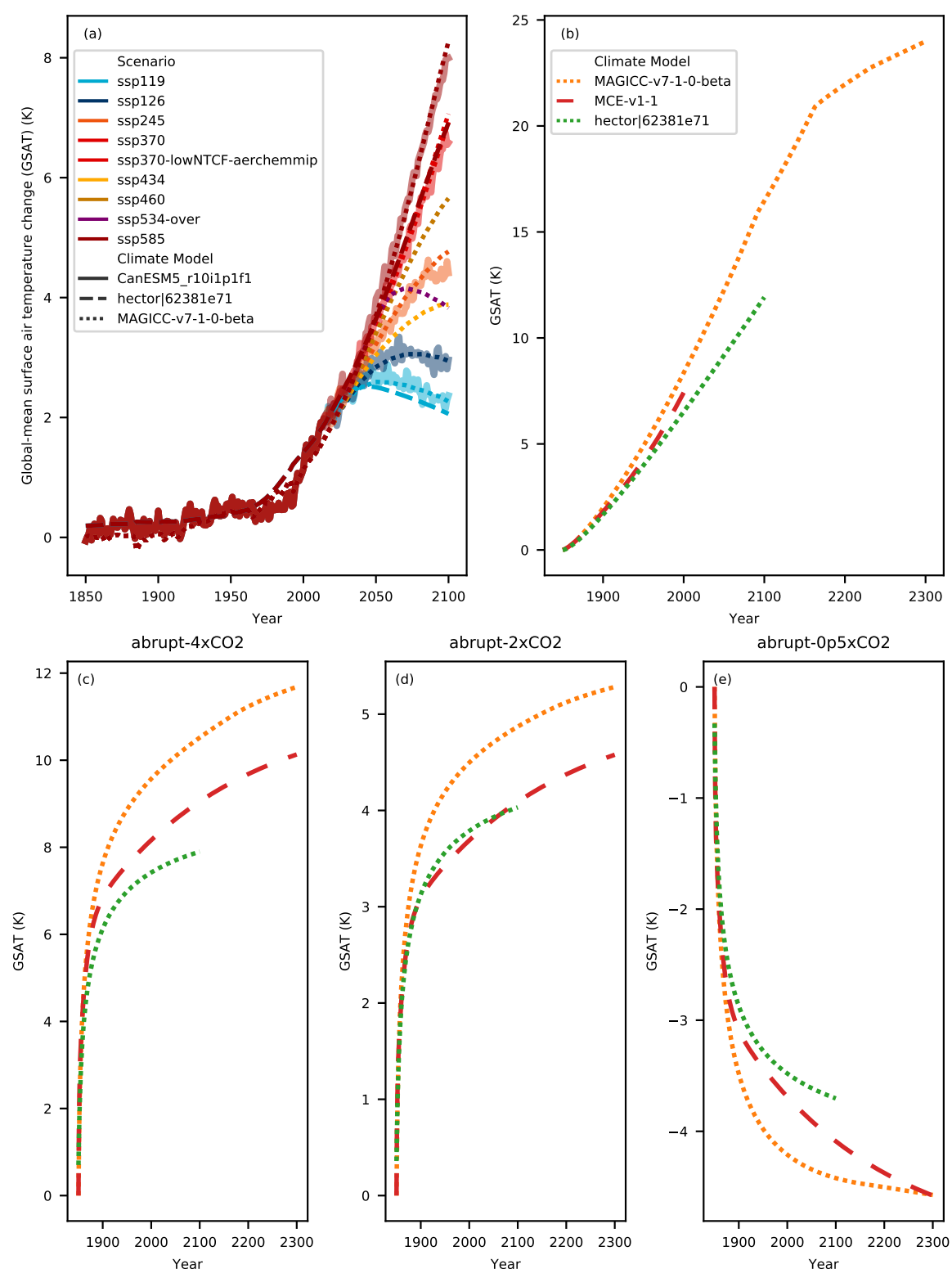

Figure S9. Emulation of CanESM5_r10i1p1f1 by RCMs in RCMIP Phase 1. The thick transparent lines are the target CMIP6 model output (here from CanESM5_r10i1p1f1). The thin lines are emulations from different RCMs. Panel (a) shows results for scenario based experiments while panels (b) - (e) show results for idealised CO2-only experiments (note that panels (b) - (e) share the same legend). 

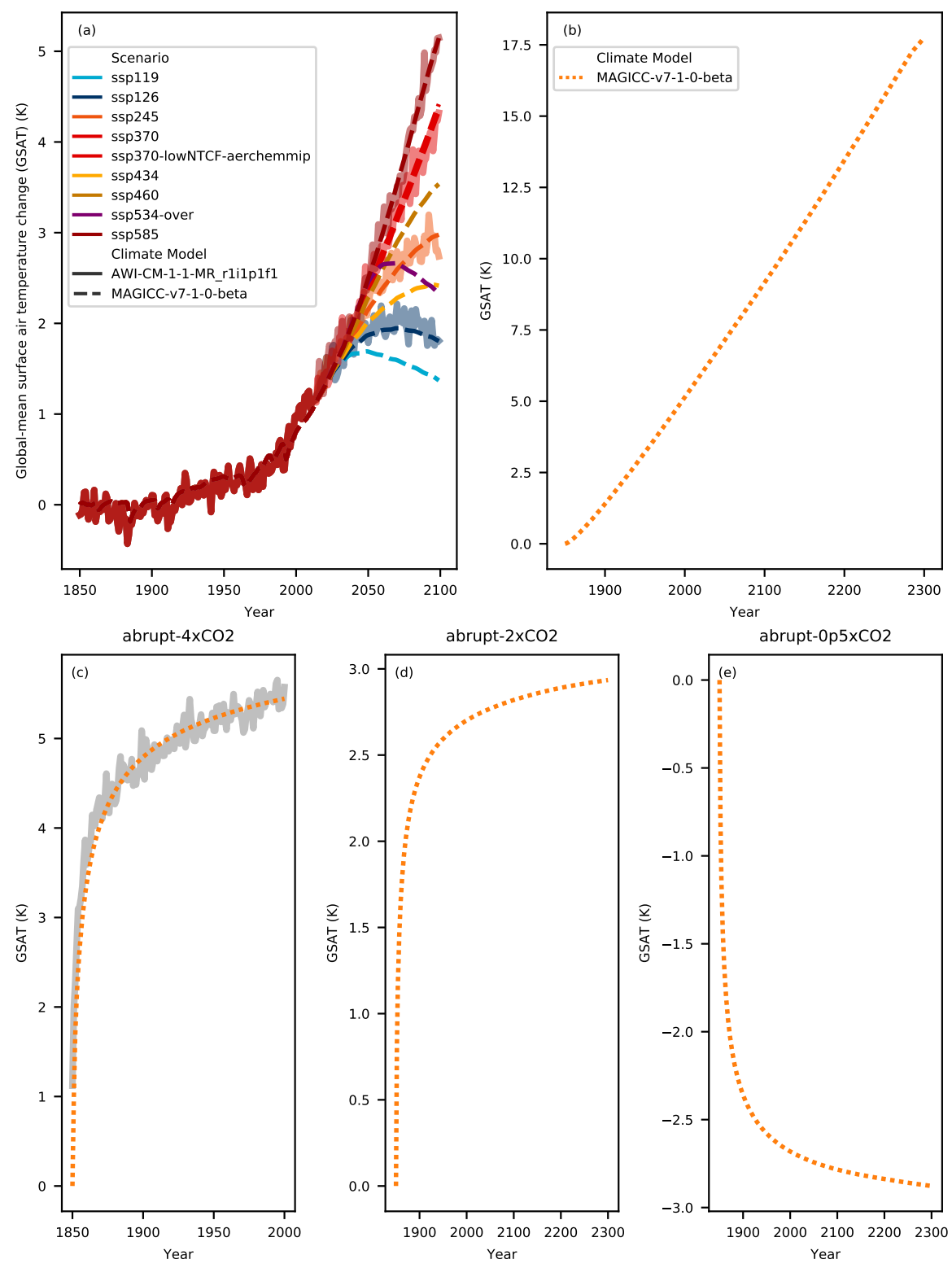

Figure S10. Emulation of generic by RCMs in RCMIP Phase 1. The thick transparent lines are the target CMIP6 model output (here from generic). The thin lines are emulations from different RCMs. Panel (a) shows results for scenario based experiments while panels (b) - (e) show results for idealised CO2-only experiments (note that panels (b) - (e) share the same legend). 

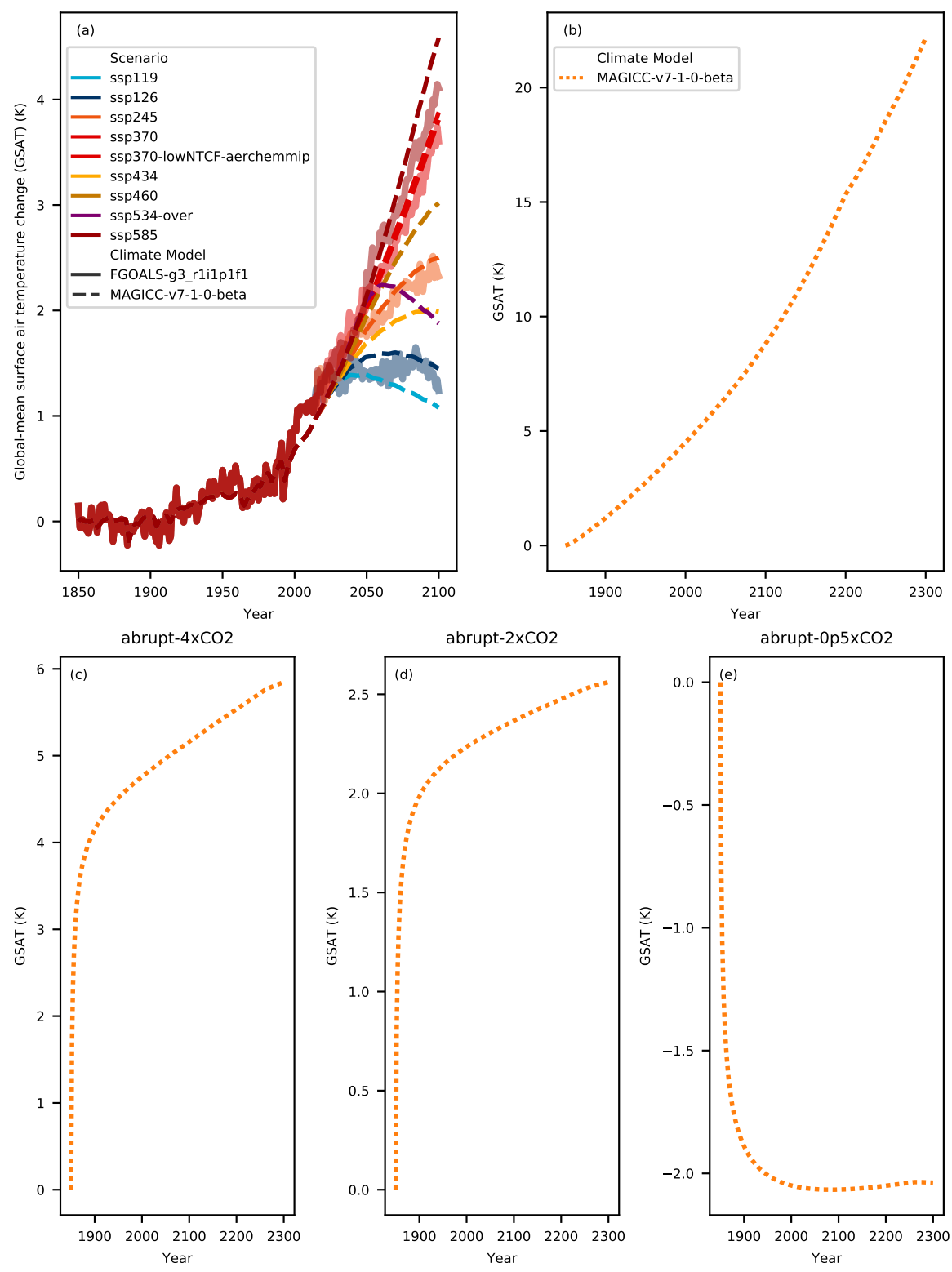

Figure S11. Emulation of FGOALS-g3_r1ilp1f1 by RCMs in RCMIP Phase 1. The thick transparent lines are the target CMIP6 model output (here from FGOALS-g3_rli1p1f1). The thin lines are emulations from different RCMs. Panel (a) shows results for scenario based experiments while panels (b) - (e) show results for idealised CO2-only experiments (note that panels (b) - (e) share the same legend). 

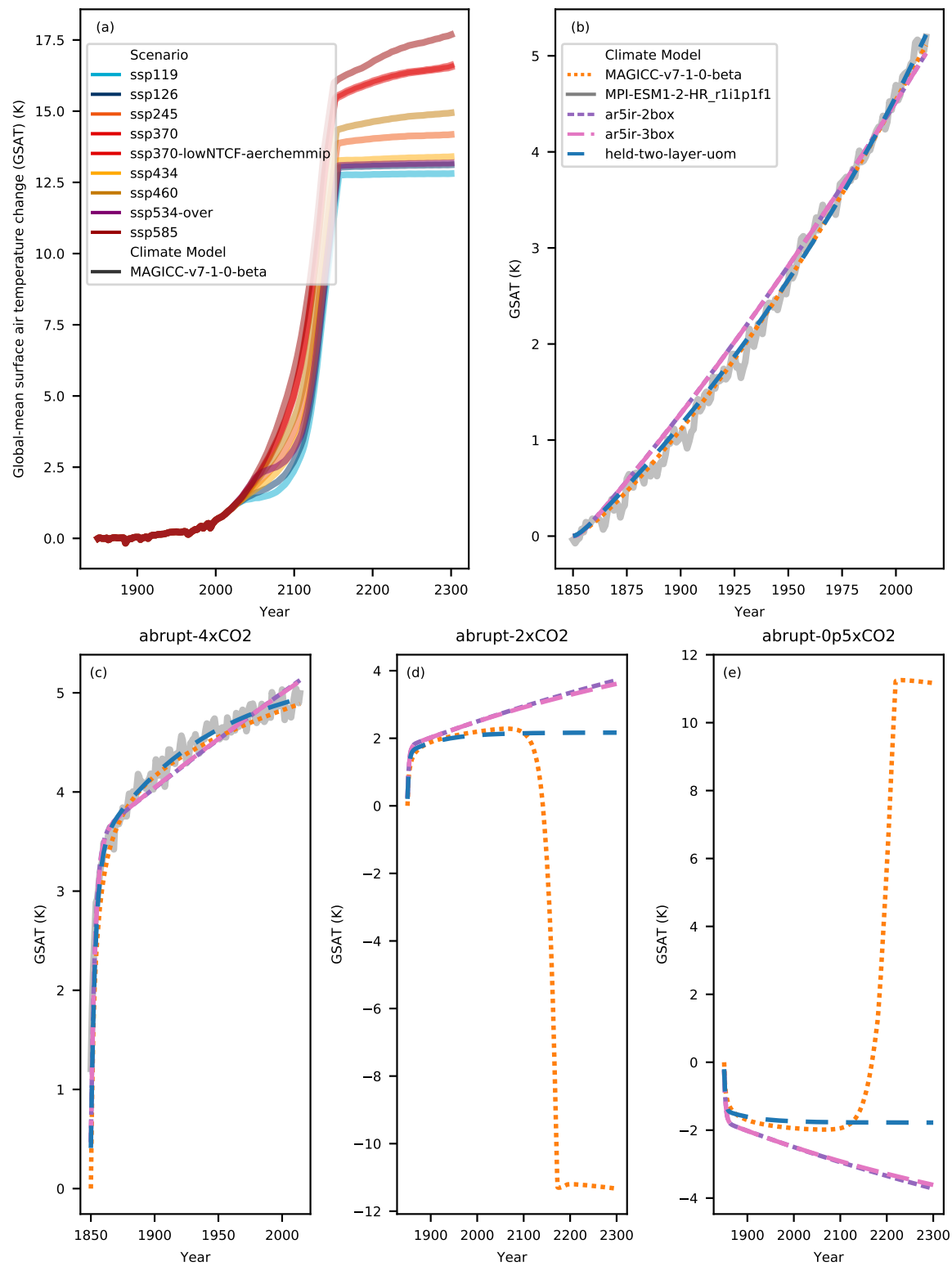

Figure S12. Emulation of MPI-ESM1-2-HR_r1i1p1f1 by RCMs in RCMIP Phase 1. The thick transparent lines are the target CMIP6 model output (here from MPI-ESM1-2-HR_r1i1p1f1). The thin lines are emulations from different RCMs. Panel (a) shows results for scenario based experiments while panels (b) - (e) show results for idealised CO2-only experiments (note that panels (b) - (e) share the same legend). 

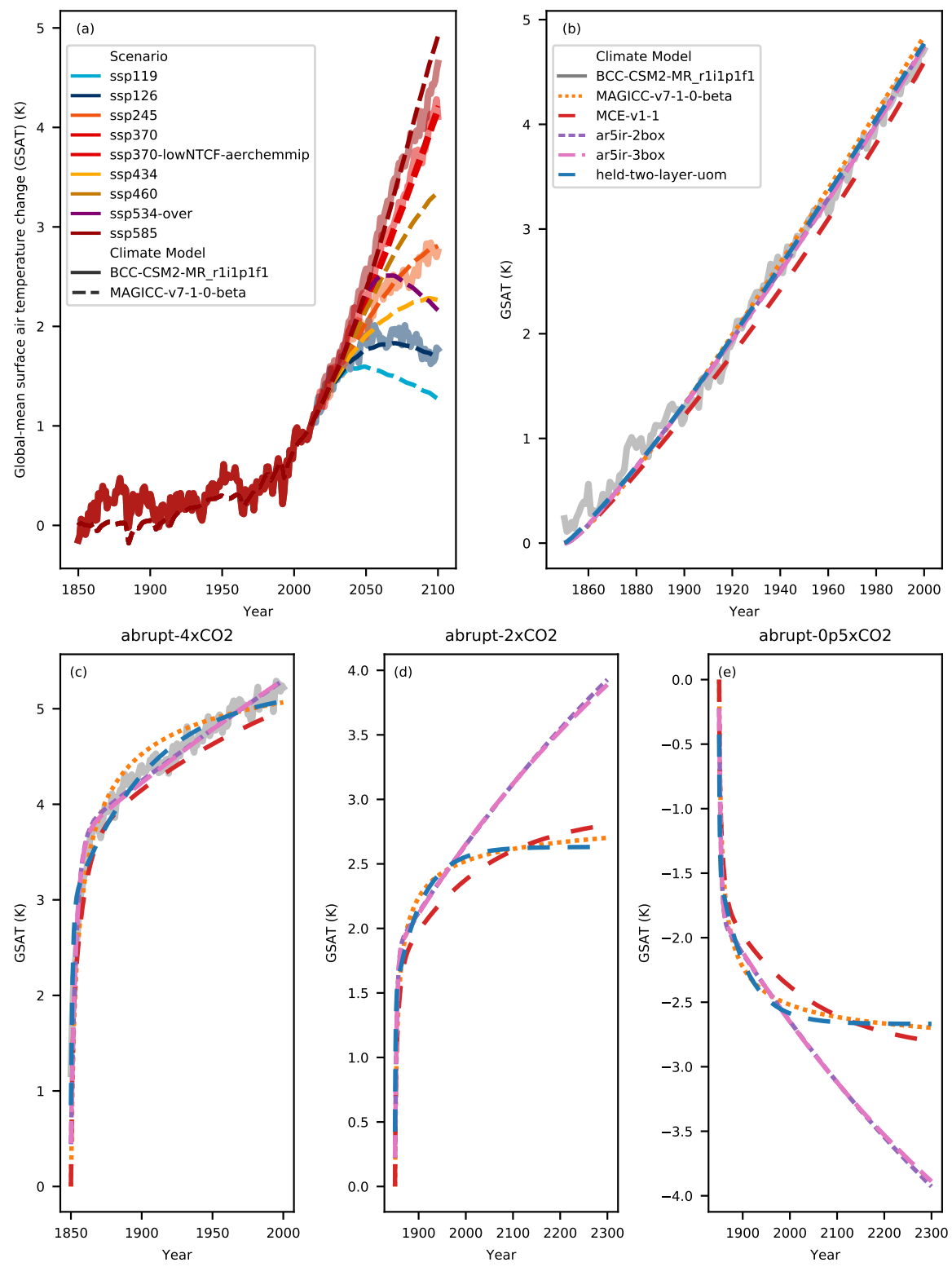

Figure S13. Emulation of BCC-CSM2-MR_r1i1p1f1 by RCMs in RCMIP Phase 1. The thick transparent lines are the target CMIP6 model output (here from BCC-CSM2-MR_r1i1p1f1). The thin lines are emulations from different RCMs. Panel (a) shows results for scenario based experiments while panels (b) - (e) show results for idealised CO2-only experiments (note that panels (b) - (e) share the same legend). 

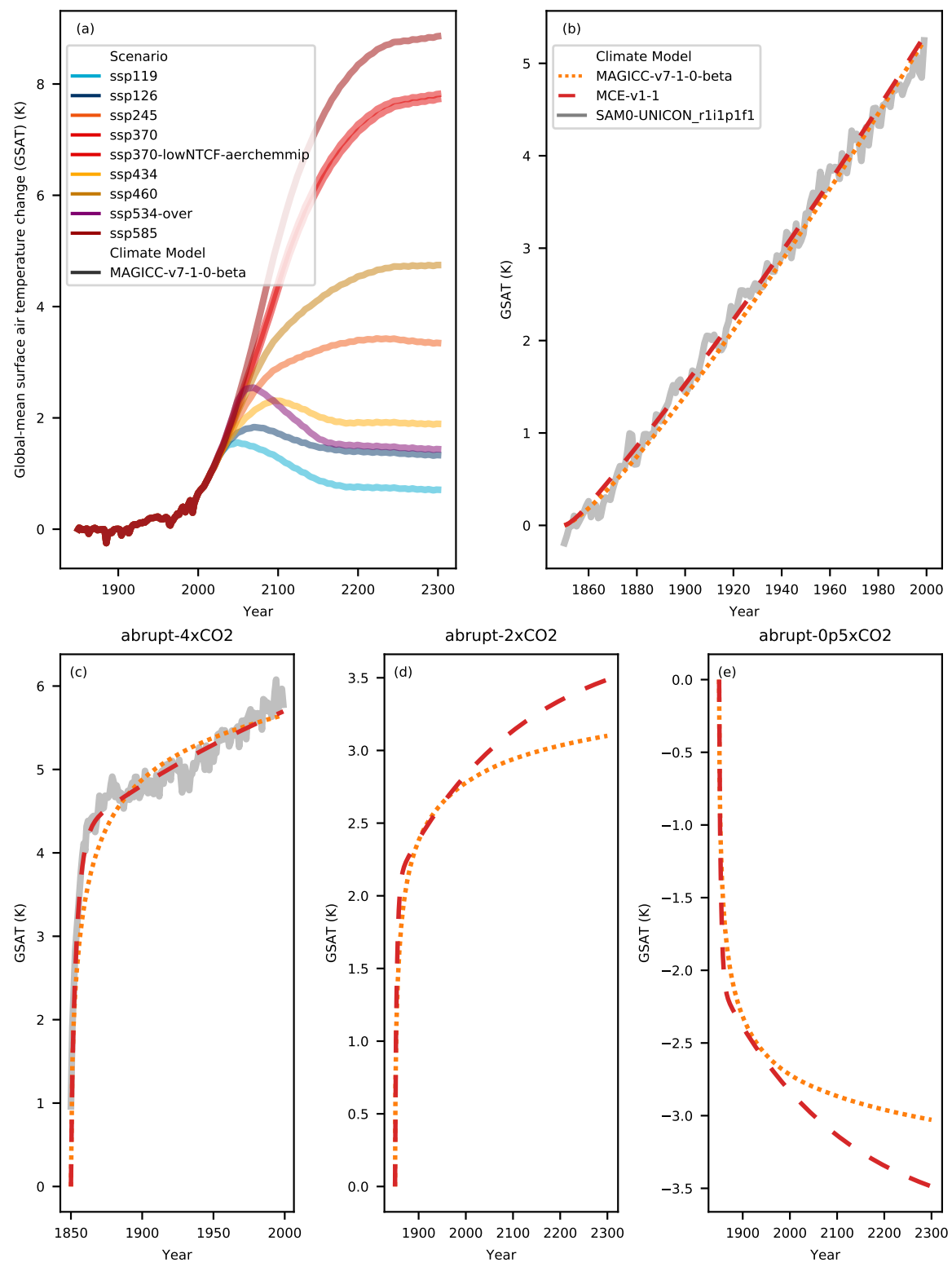

Figure S14. Emulation of SAM0-UNICON_r1i1p1f1 by RCMs in RCMIP Phase 1. The thick transparent lines are the target CMIP6 model output (here from SAM0-UNICON_r1i1p1f1). The thin lines are emulations from different RCMs. Panel (a) shows results for scenario based experiments while panels (b) - (e) show results for idealised CO2-only experiments (note that panels (b) - (e) share the same legend). 

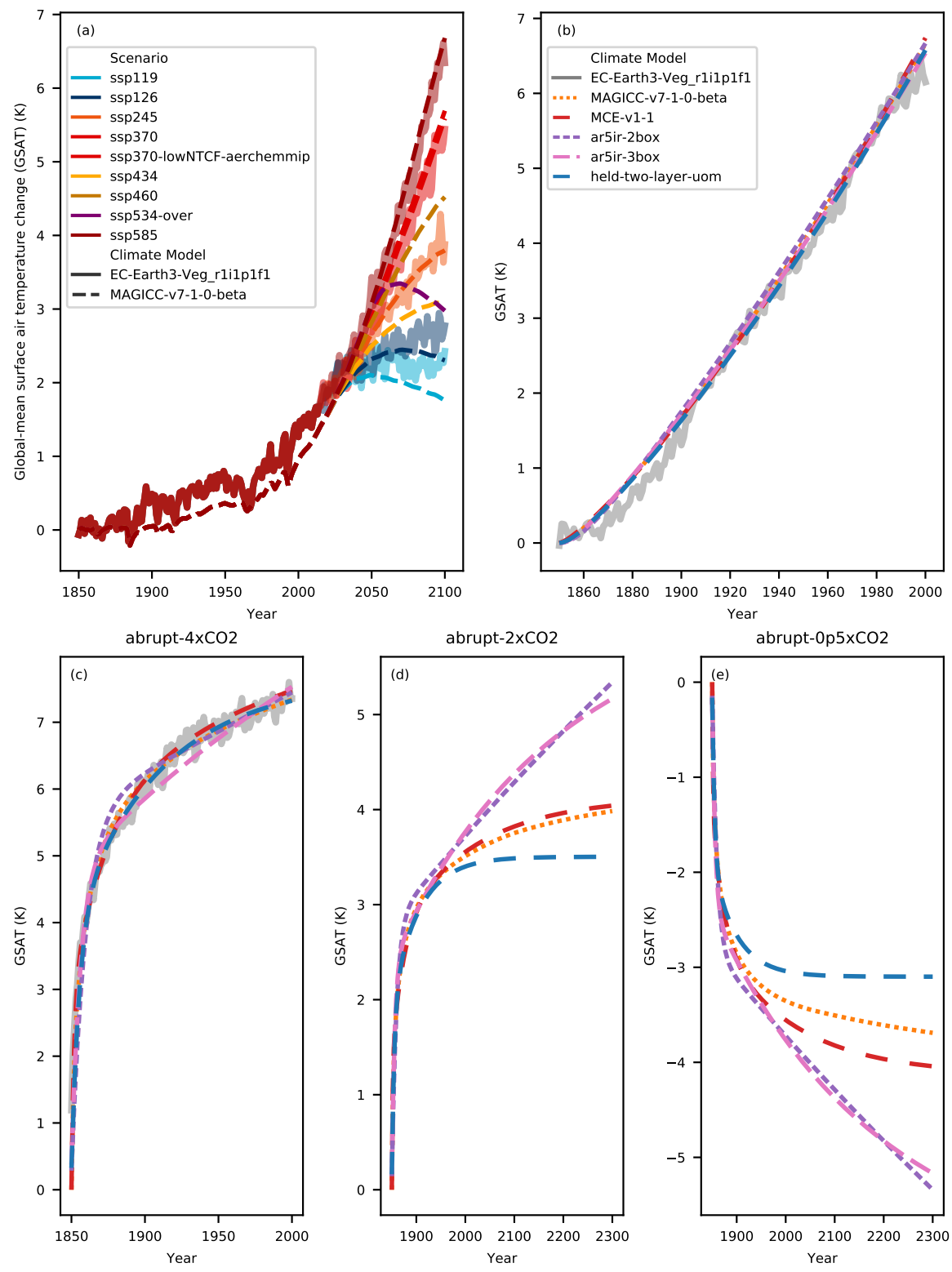

Figure S15. Emulation of EC-Earth3-Veg_r1i1p1f1 by RCMs in RCMIP Phase 1. The thick transparent lines are the target CMIP6 model output (here from EC-Earth3-Veg_r1i1p1f1). The thin lines are emulations from different RCMs. Panel (a) shows results for scenario based experiments while panels (b) - (e) show results for idealised CO2-only experiments (note that panels (b) - (e) share the same legend). 

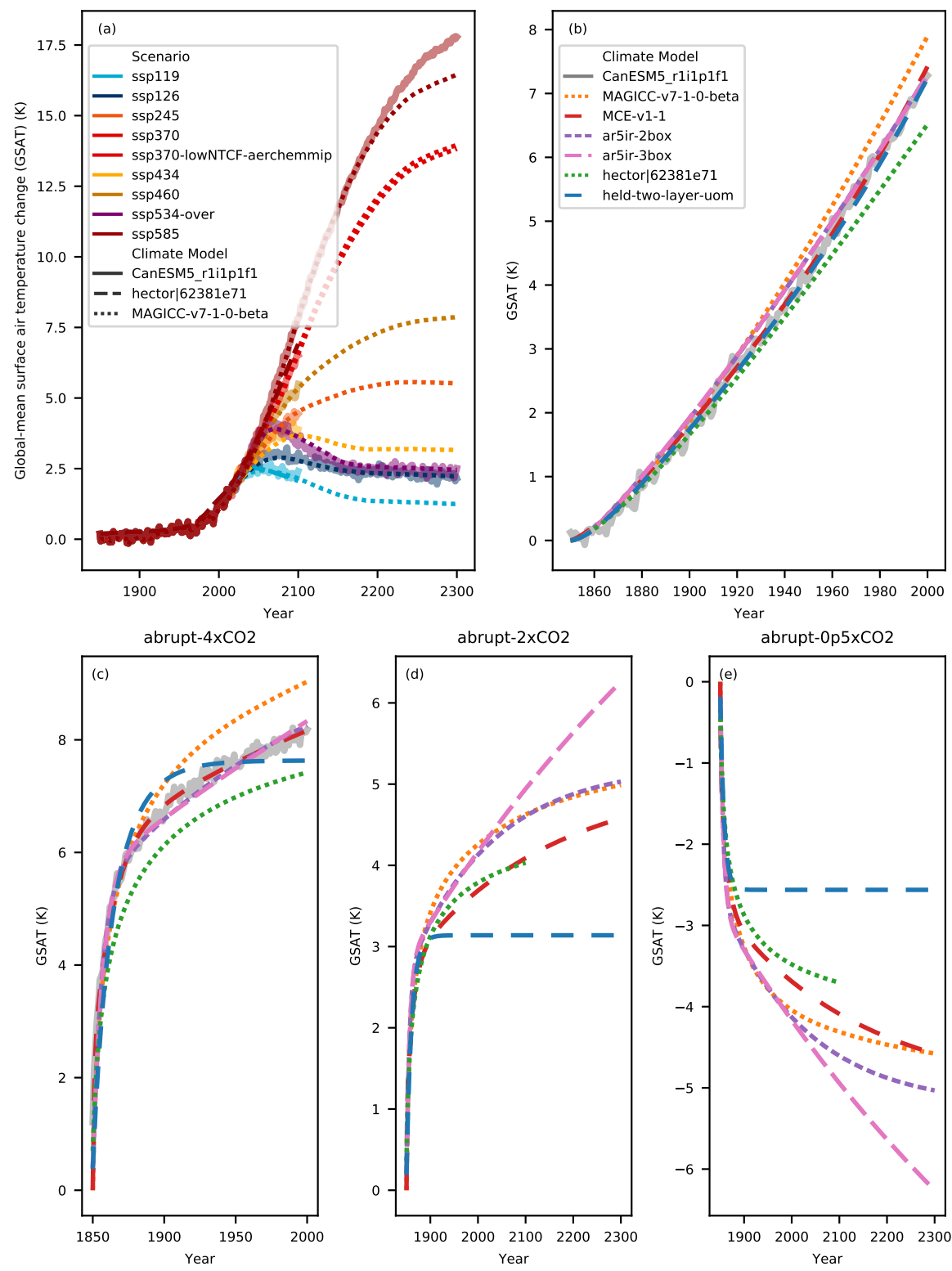

Figure S16. Emulation of CanESM5_r1i1p1f1 by RCMs in RCMIP Phase 1. The thick transparent lines are the target CMIP6 model output (here from CanESM5_r1i1p1f1). The thin lines are emulations from different RCMs. Panel (a) shows results for scenario based experiments while panels (b) - (e) show results for idealised CO2-only experiments (note that panels (b) - (e) share the same legend). 

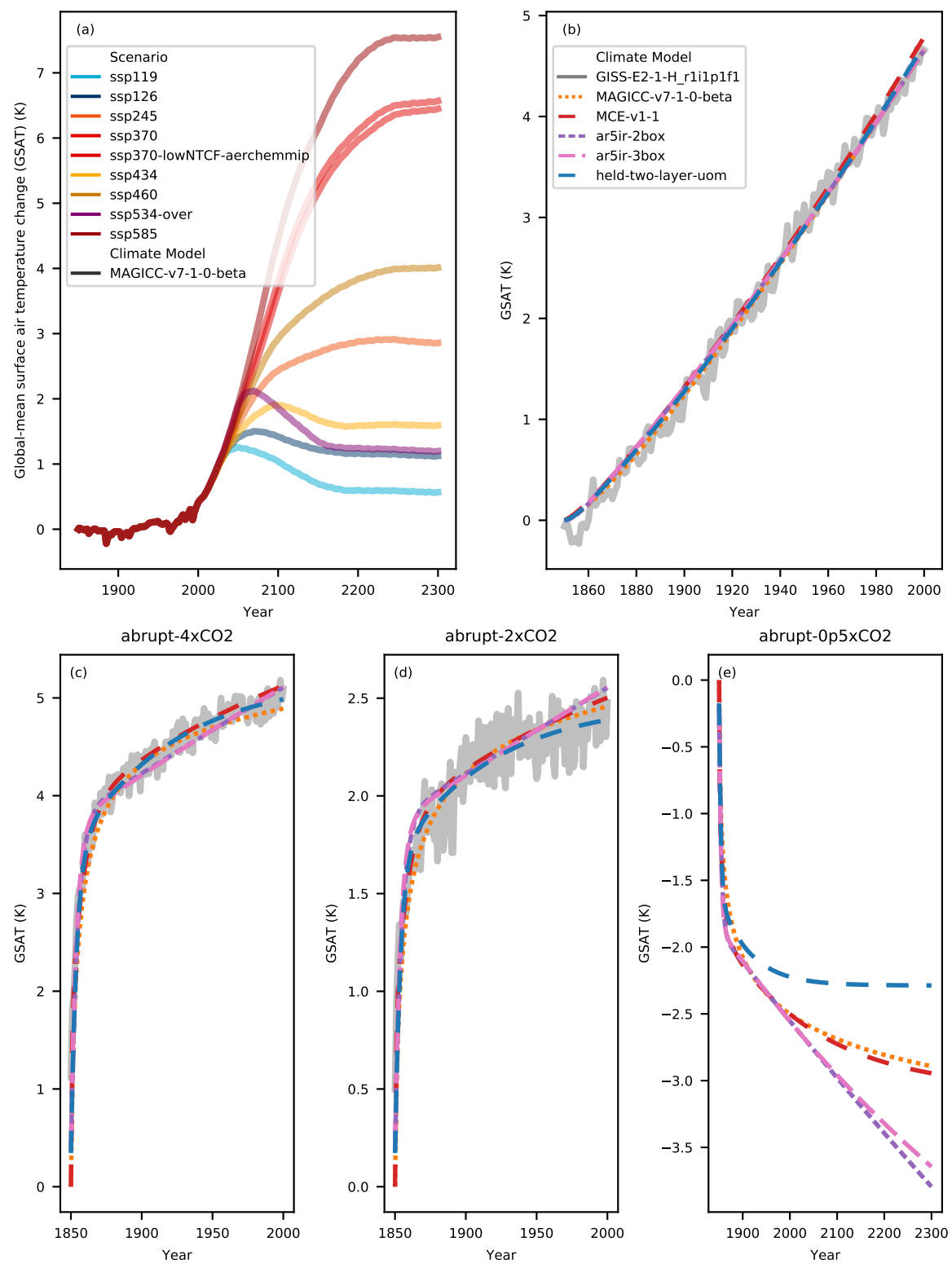

Figure S17. Emulation of GISS-E2-1-H_r1i1p1f1 by RCMs in RCMIP Phase 1. The thick transparent lines are the target CMIP6 model output (here from GISS-E2-1-H_r1i1p1f1). The thin lines are emulations from different RCMs. Panel (a) shows results for scenario based experiments while panels (b) - (e) show results for idealised CO2-only experiments (note that panels (b) - (e) share the same legend). 

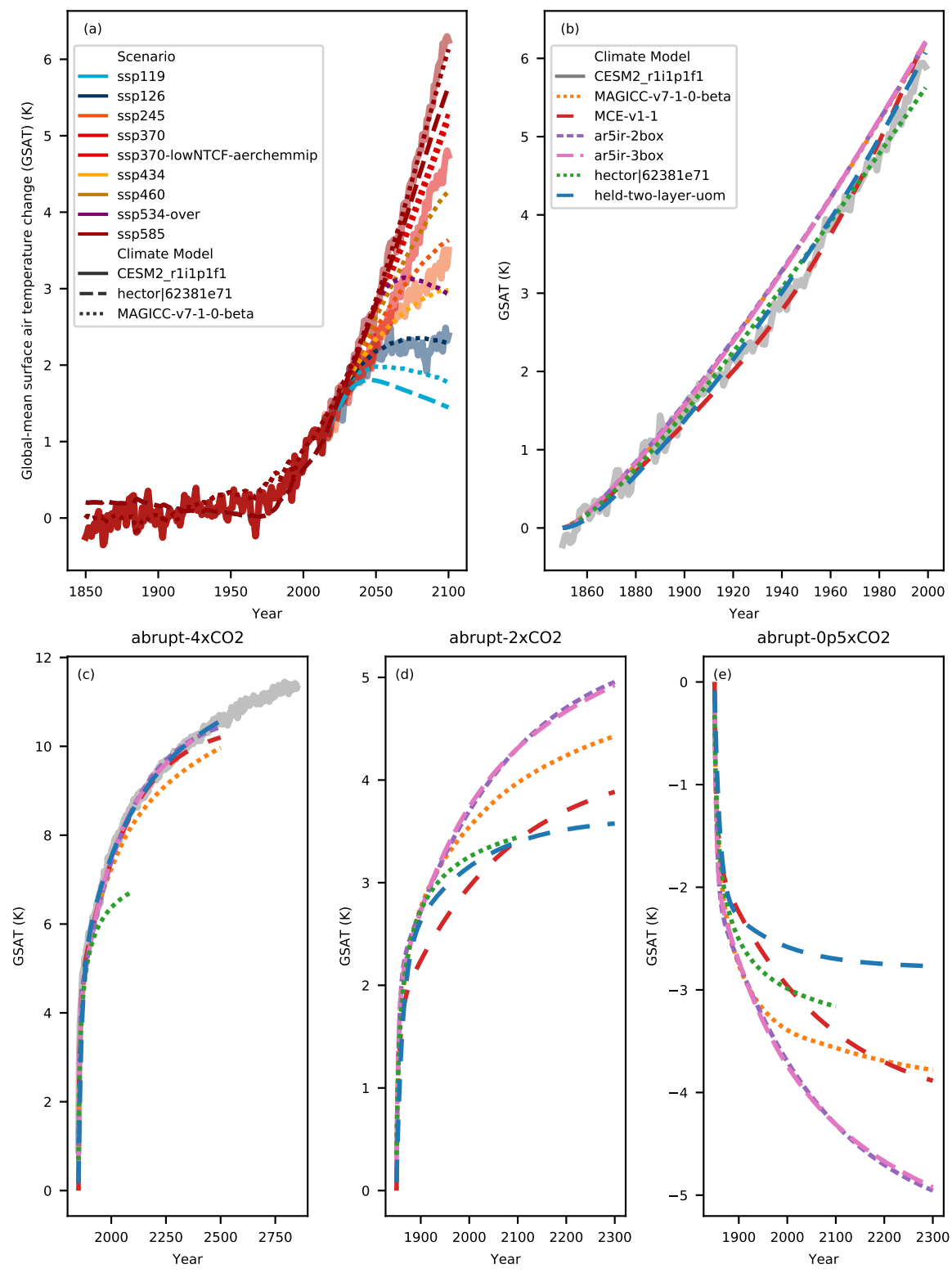

Figure S18. Emulation of CESM2_r1i1p1f1 by RCMs in RCMIP Phase 1. The thick transparent lines are the target CMIP6 model output (here from CESM2_r1i1p1f1). The thin lines are emulations from different RCMs. Panel (a) shows results for scenario based experiments while panels (b) - (e) show results for idealised CO2-only experiments (note that panels (b) - (e) share the same legend). 

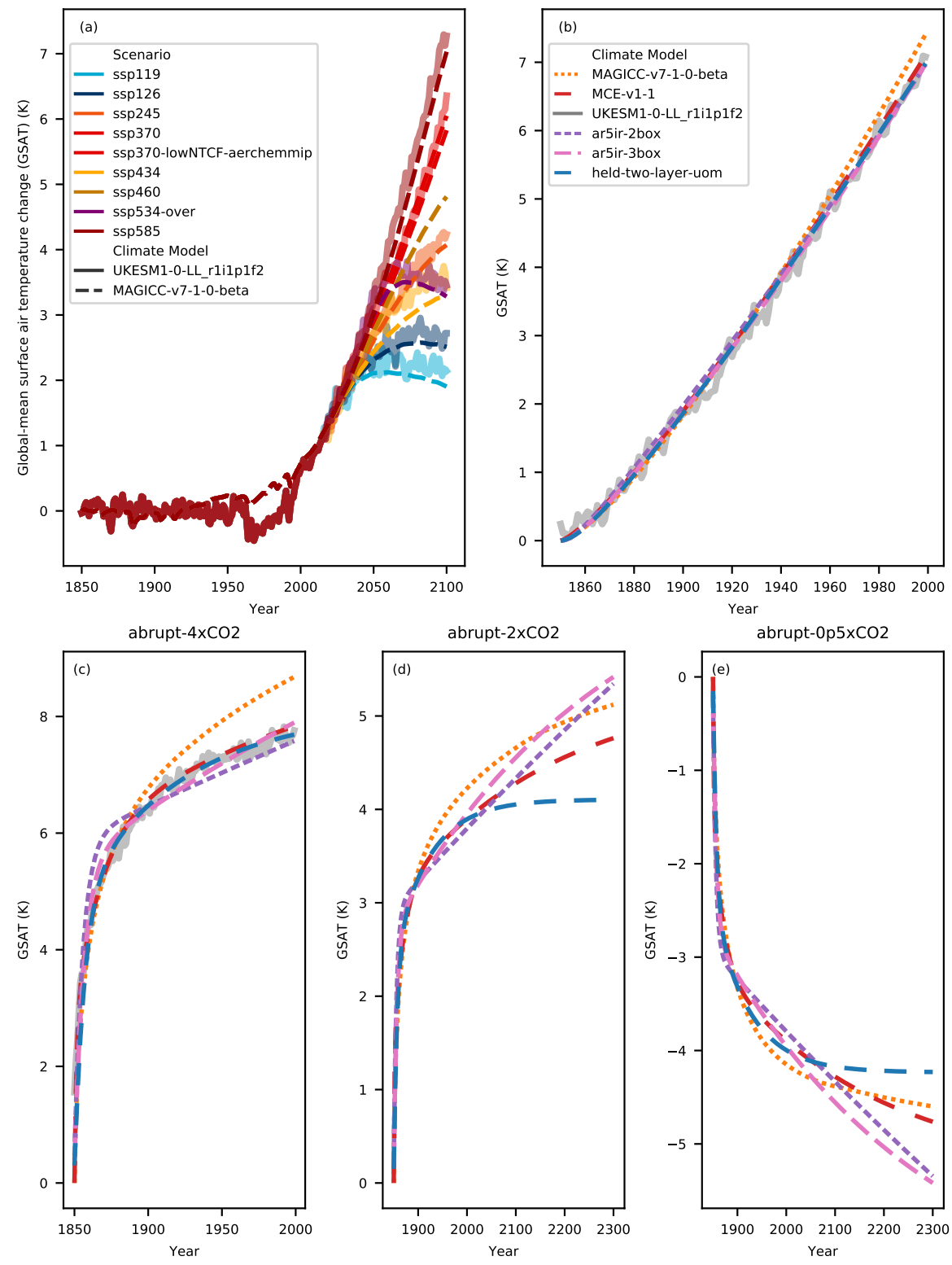

Figure S19. Emulation of UKESM1-0-LL_r1i1p1f2 by RCMs in RCMIP Phase 1. The thick transparent lines are the target CMIP6 model output (here from UKESM1-0-LL_r1i1p1f2). The thin lines are emulations from different RCMs. Panel (a) shows results for scenario based experiments while panels (b) - (e) show results for idealised CO2-only experiments (note that panels (b) - (e) share the same legend). 

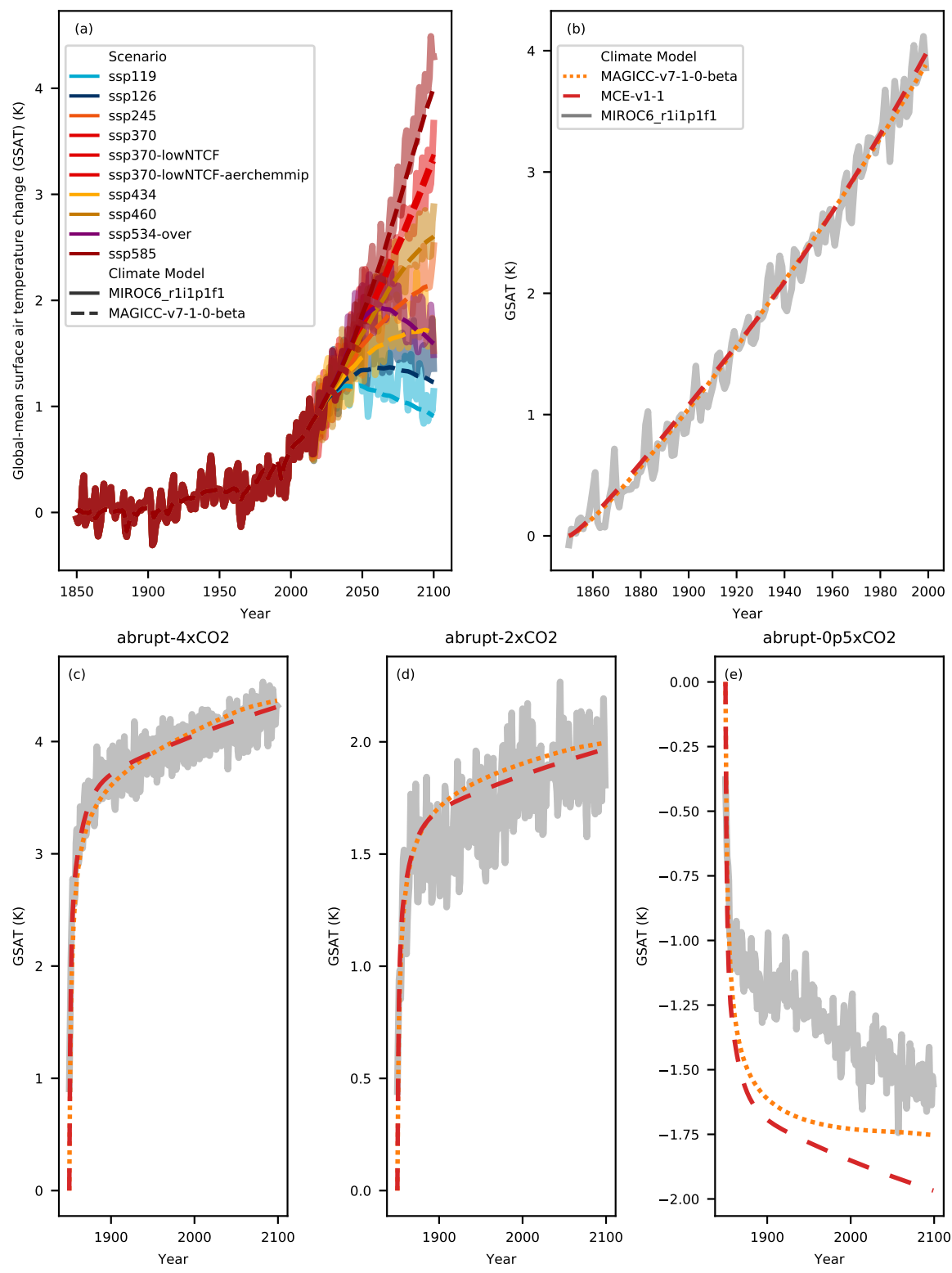

Figure S20. Emulation of MIROC6_r1i1p1f1 by RCMs in RCMIP Phase 1. The thick transparent lines are the target CMIP6 model output (here from MIROC6_r1i1p1f1). The thin lines are emulations from different RCMs. Panel (a) shows results for scenario based experiments while panels (b) - (e) show results for idealised CO2-only experiments (note that panels (b) - (e) share the same legend). 

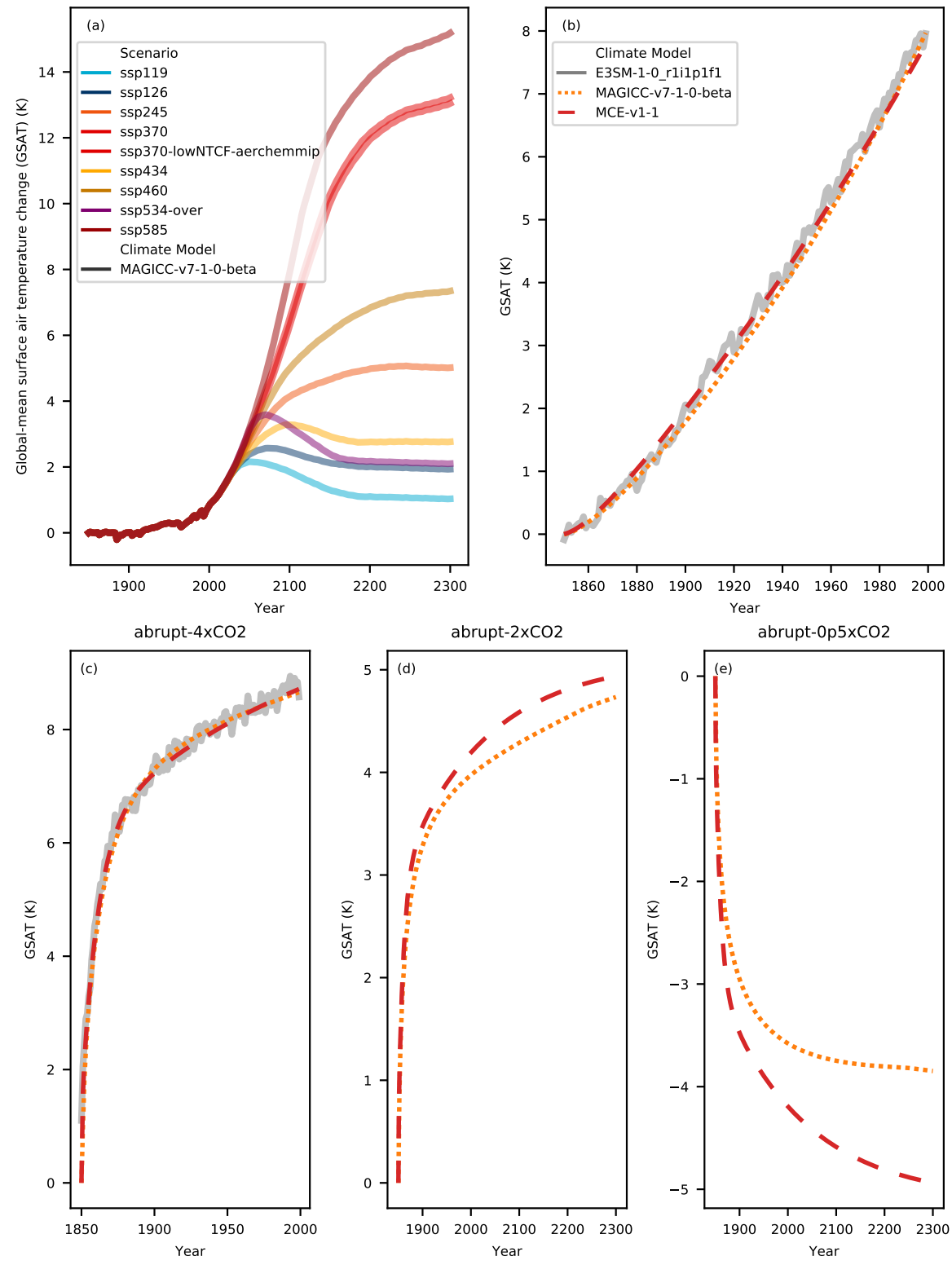

Figure S21. Emulation of E3SM-1-0_r1i1p1f1 by RCMs in RCMIP Phase 1. The thick transparent lines are the target CMIP6 model output (here from E3SM-1-0_r1i1p1f1). The thin lines are emulations from different RCMs. Panel (a) shows results for scenario based experiments while panels (b) - (e) show results for idealised CO2-only experiments (note that panels (b) - (e) share the same legend). 

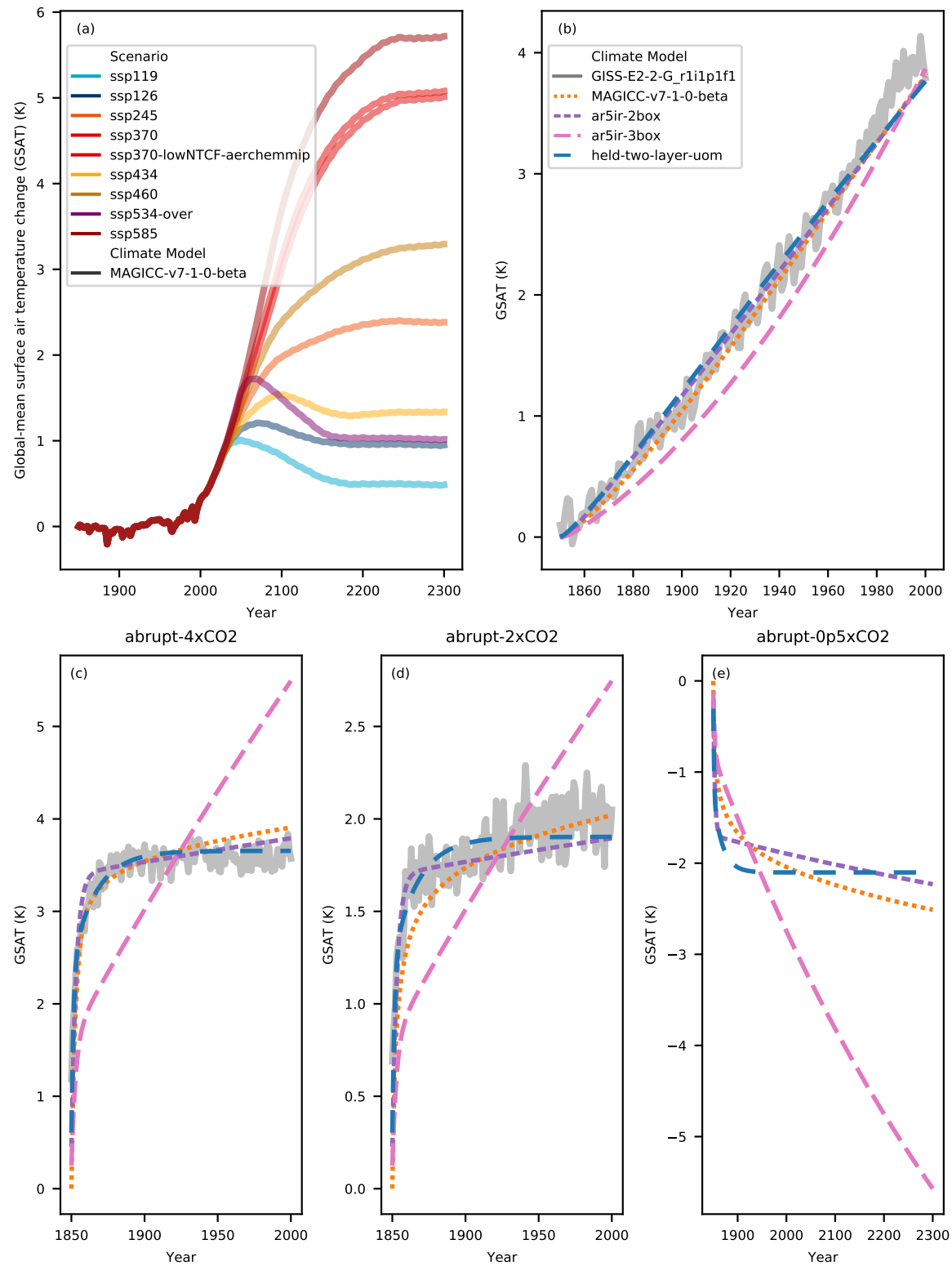

Figure S22. Emulation of GISS-E2-2-G_r1i1p1f1 by RCMs in RCMIP Phase 1. The thick transparent lines are the target CMIP6 model output (here from GISS-E2-2-G_r1i1p1f1). The thin lines are emulations from different RCMs. Panel (a) shows results for scenario based experiments while panels (b) - (e) show results for idealised CO2-only experiments (note that panels (b) - (e) share the same legend). 

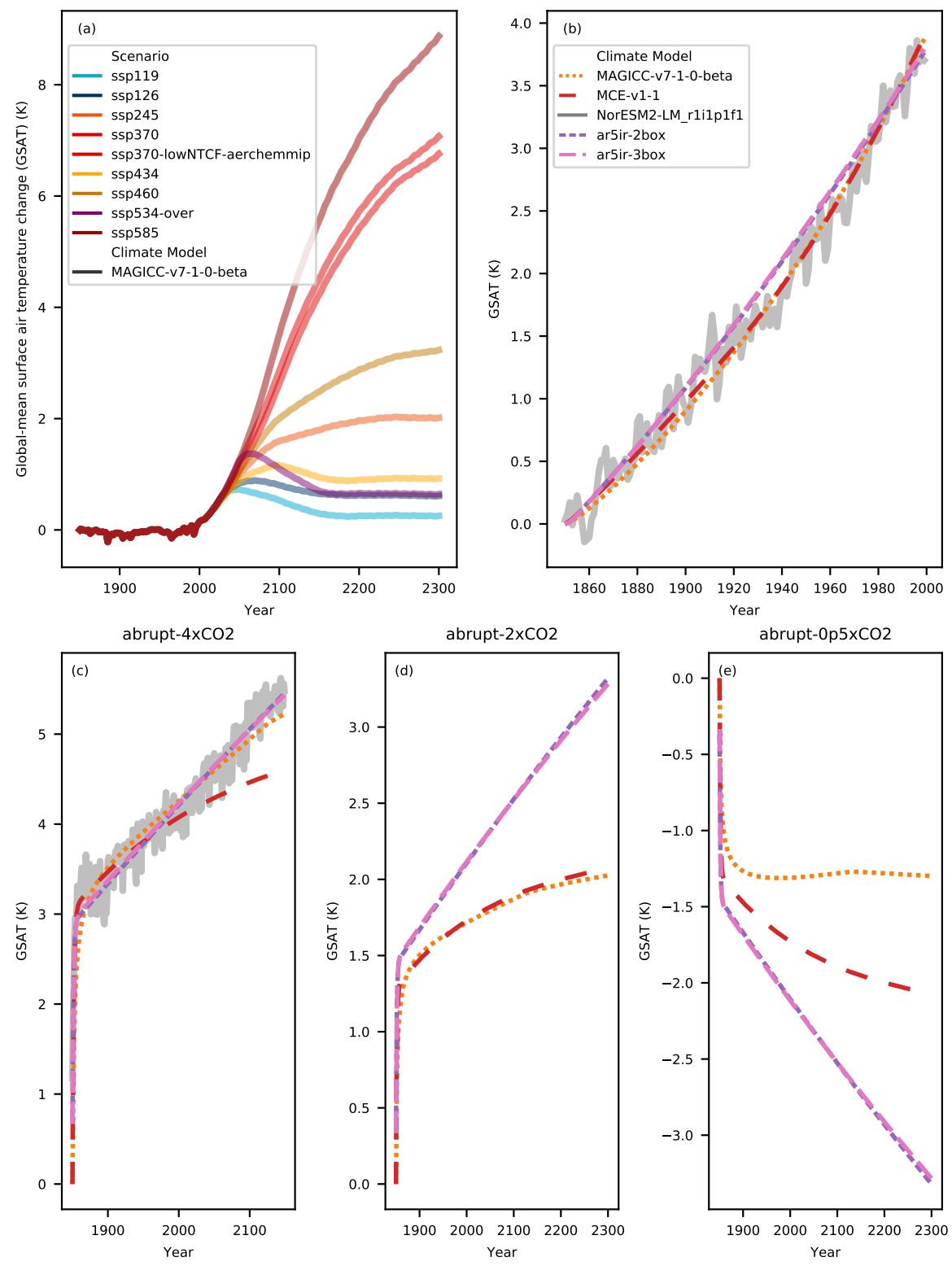

Figure S23. Emulation of NorESM2-LM_r1i1p1f1 by RCMs in RCMIP Phase 1. The thick transparent lines are the target CMIP6 model output (here from NorESM2-LM_r1ilp1f1). The thin lines are emulations from different RCMs. Panel (a) shows results for scenario based experiments while panels (b) - (e) show results for idealised CO2-only experiments (note that panels (b) - (e) share the same legend). 

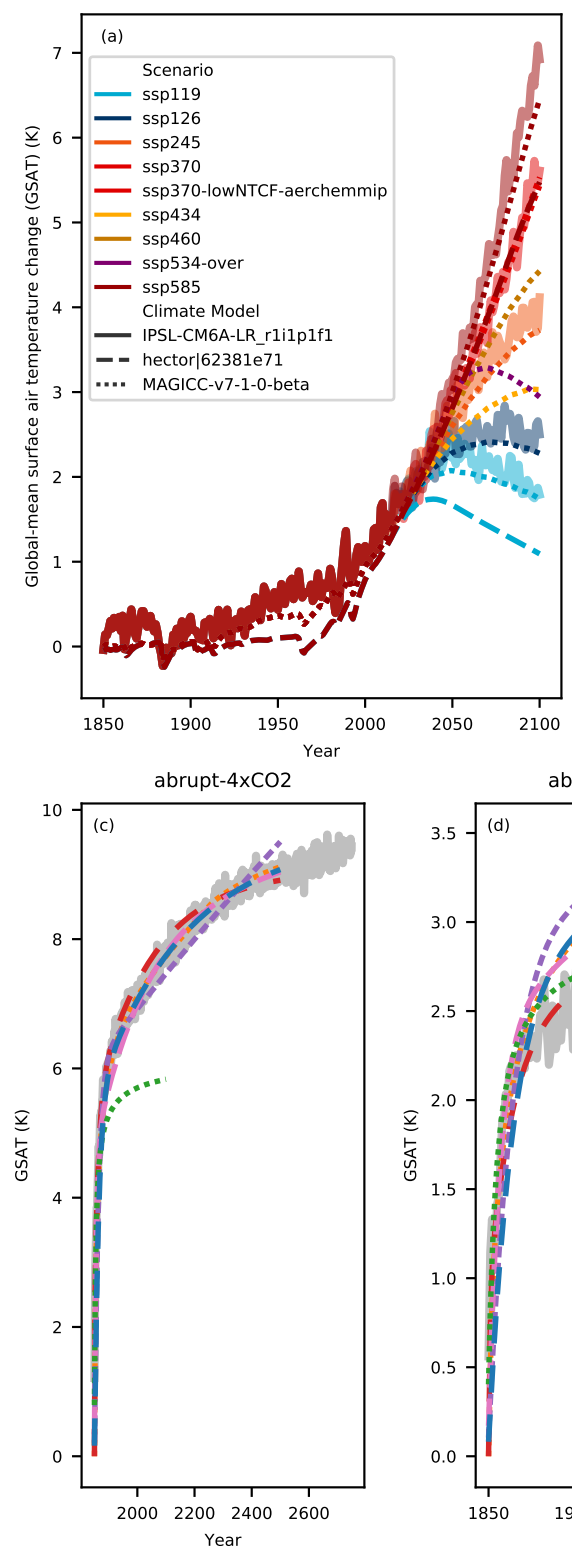

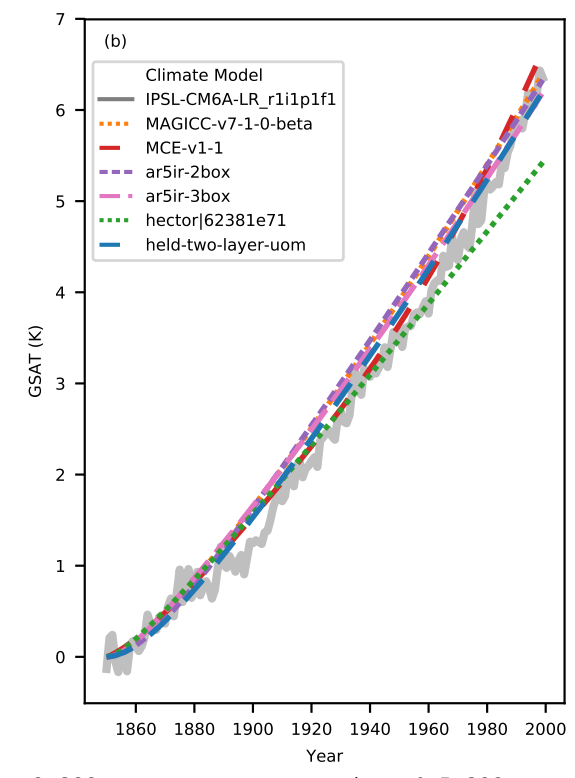

abrupt-0p5xCO2
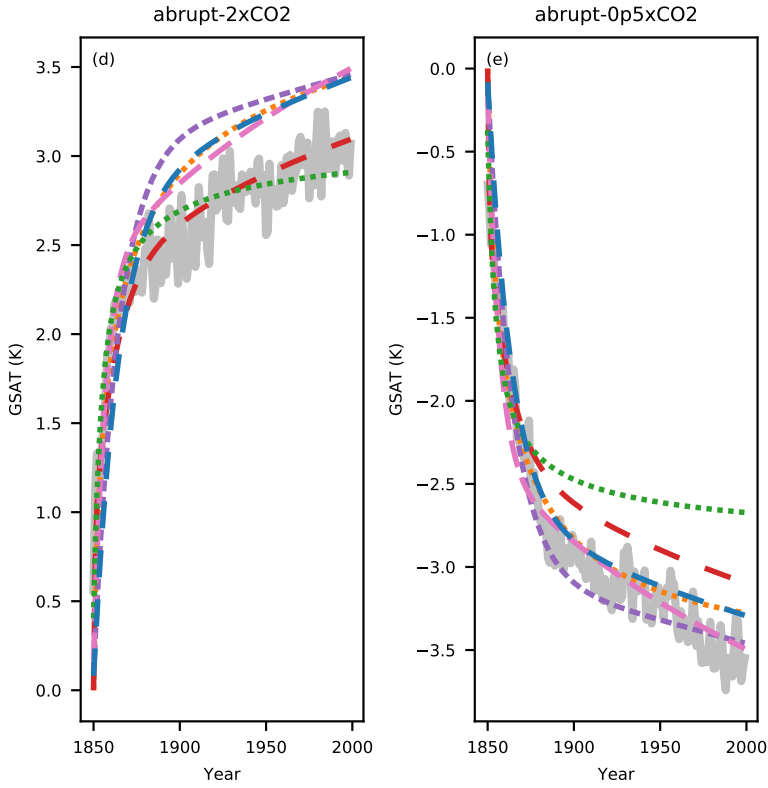

Figure S24. Emulation of IPSL-CM6A-LR_r1i1p1f1 by RCMs in RCMIP Phase 1. The thick transparent lines are the target CMIP6 model output (here from IPSL-CM6A-LR_r1ilp1f1). The thin lines are emulations from different RCMs. Panel (a) shows results for scenario based experiments while panels (b) - (e) show results for idealised CO2-only experiments (note that panels (b) - (e) share the same legend). 

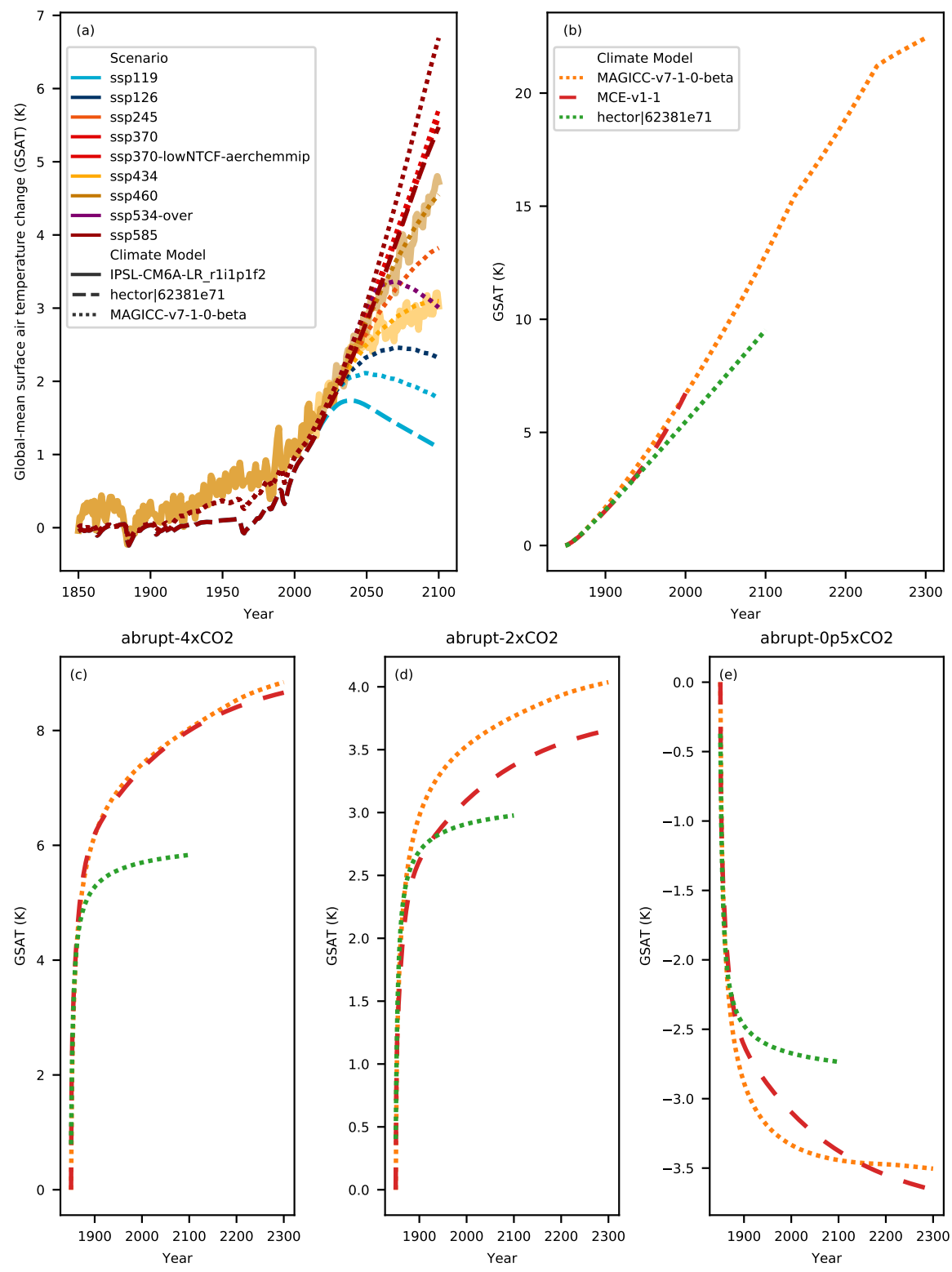

Figure S25. Emulation of IPSL-CM6A-LR_r1i1p1f2 by RCMs in RCMIP Phase 1. The thick transparent lines are the target CMIP6 model output (here from IPSL-CM6A-LR_r1i1p1f2). The thin lines are emulations from different RCMs. Panel (a) shows results for scenario based experiments while panels (b) - (e) show results for idealised CO2-only experiments (note that panels (b) - (e) share the same legend). 

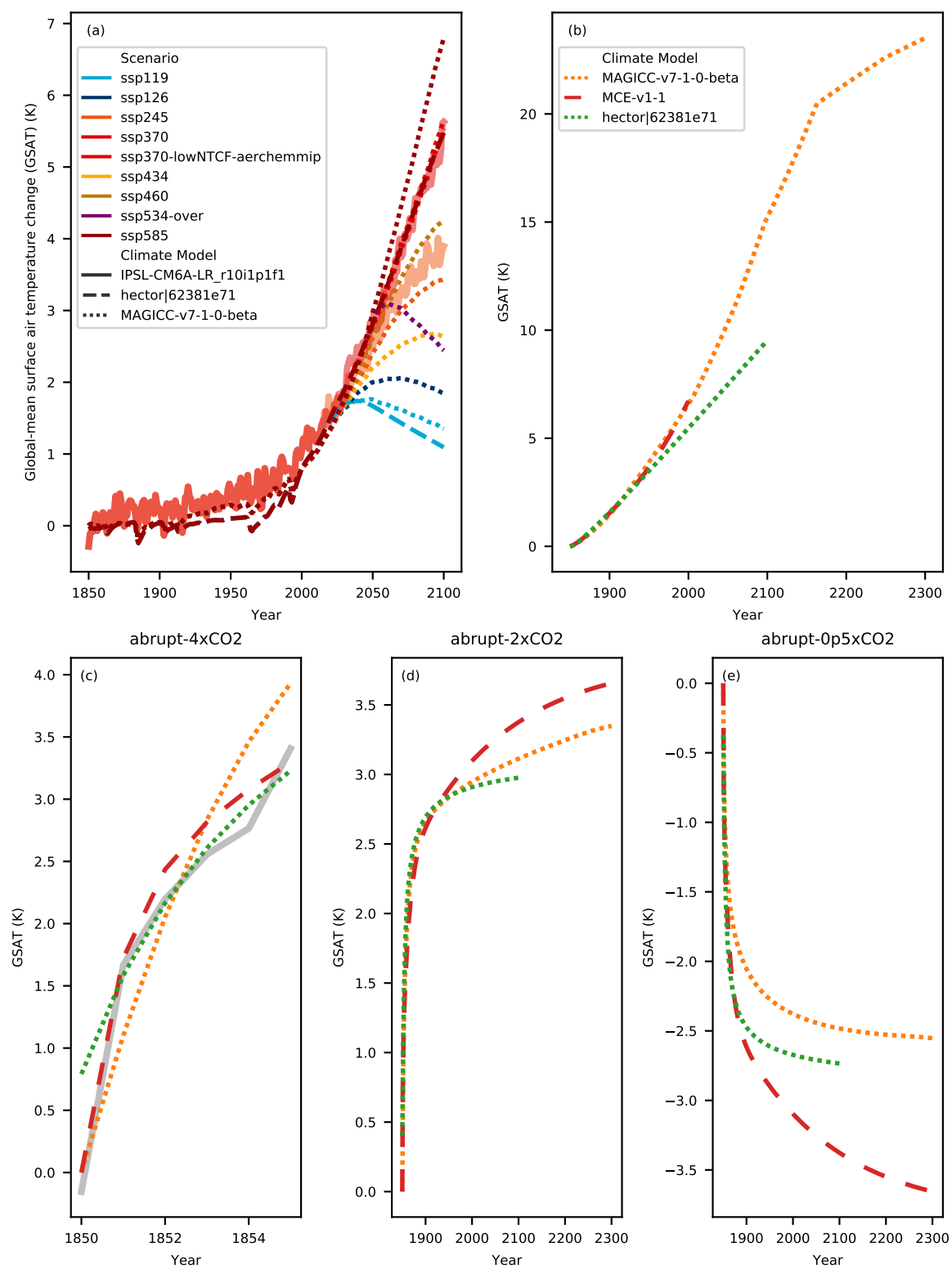

Figure S26. Emulation of IPSL-CM6A-LR_r10i1p1f1 by RCMs in RCMIP Phase 1. The thick transparent lines are the target CMIP6 model output (here from IPSL-CM6A-LR_r10i1p1f1). The thin lines are emulations from different RCMs. Panel (a) shows results for scenario based experiments while panels (b) - (e) show results for idealised CO2-only experiments (note that panels (b) - (e) share the same legend). 

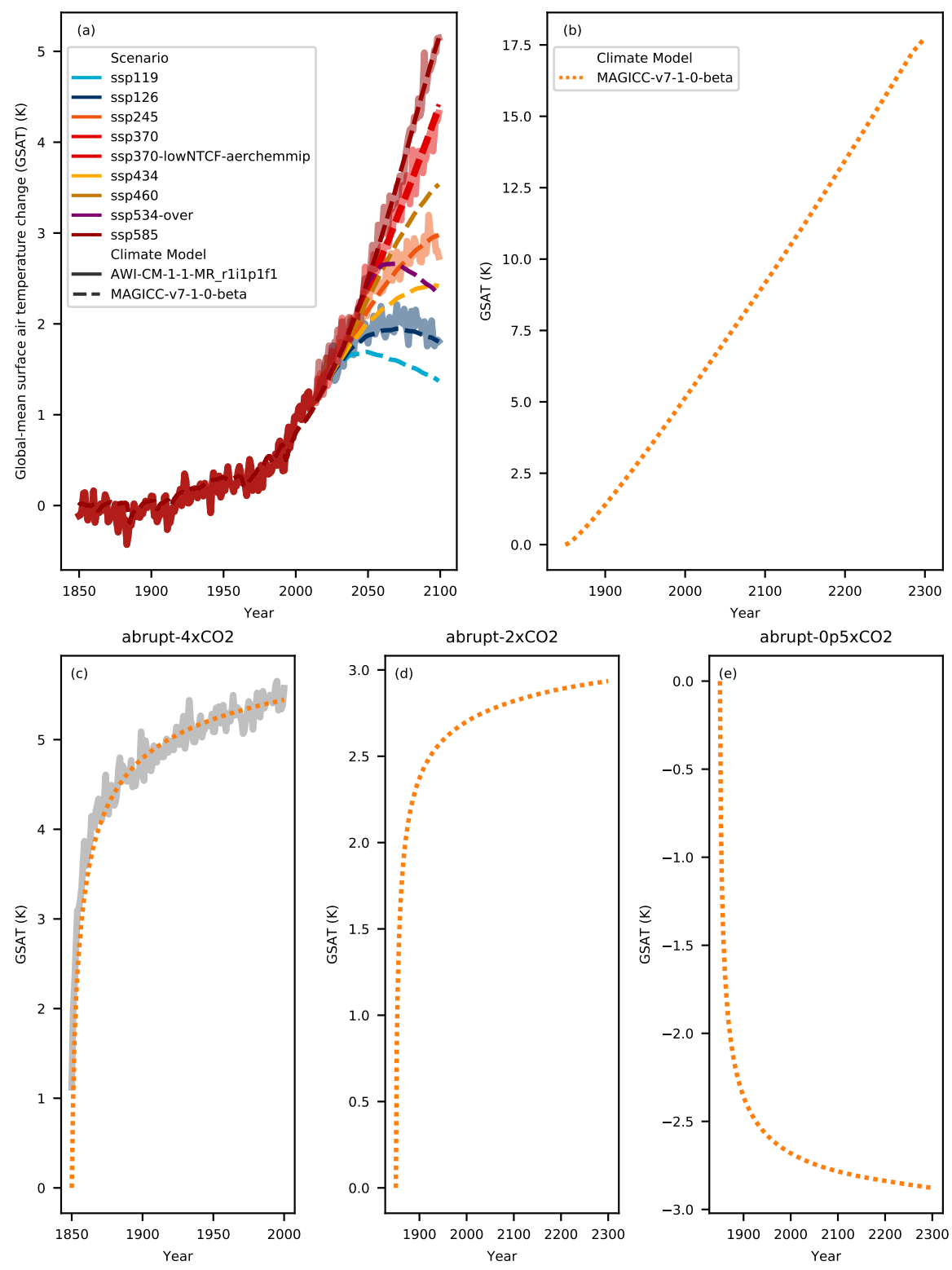

Figure S27. Emulation of AWI-CM-1-1-MR_r1i1p1f1 by RCMs in RCMIP Phase 1. The thick transparent lines are the target CMIP6 model output (here from AWI-CM-1-1-MR_r1i1p1f1). The thin lines are emulations from different RCMs. Panel (a) shows results for scenario based experiments while panels (b) - (e) show results for idealised CO2-only experiments (note that panels (b) - (e) share the same legend). 

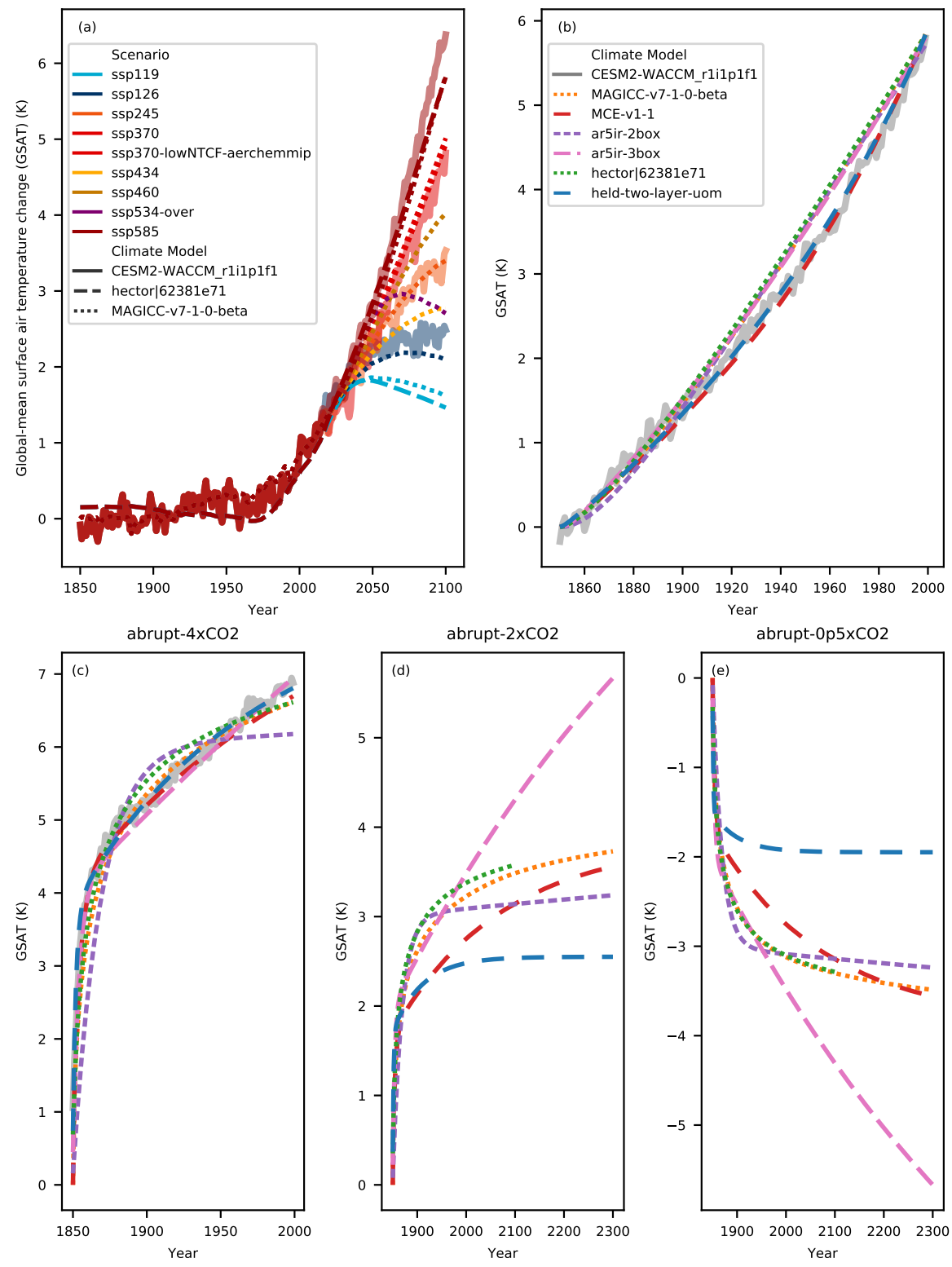

Figure S28. Emulation of CESM2-WACCM_r1i1p1f1 by RCMs in RCMIP Phase 1. The thick transparent lines are the target CMIP6 model output (here from CESM2-WACCM_r1i1p1f1). The thin lines are emulations from different RCMs. Panel (a) shows results for scenario based experiments while panels (b) - (e) show results for idealised CO2-only experiments (note that panels (b) - (e) share the same legend). 

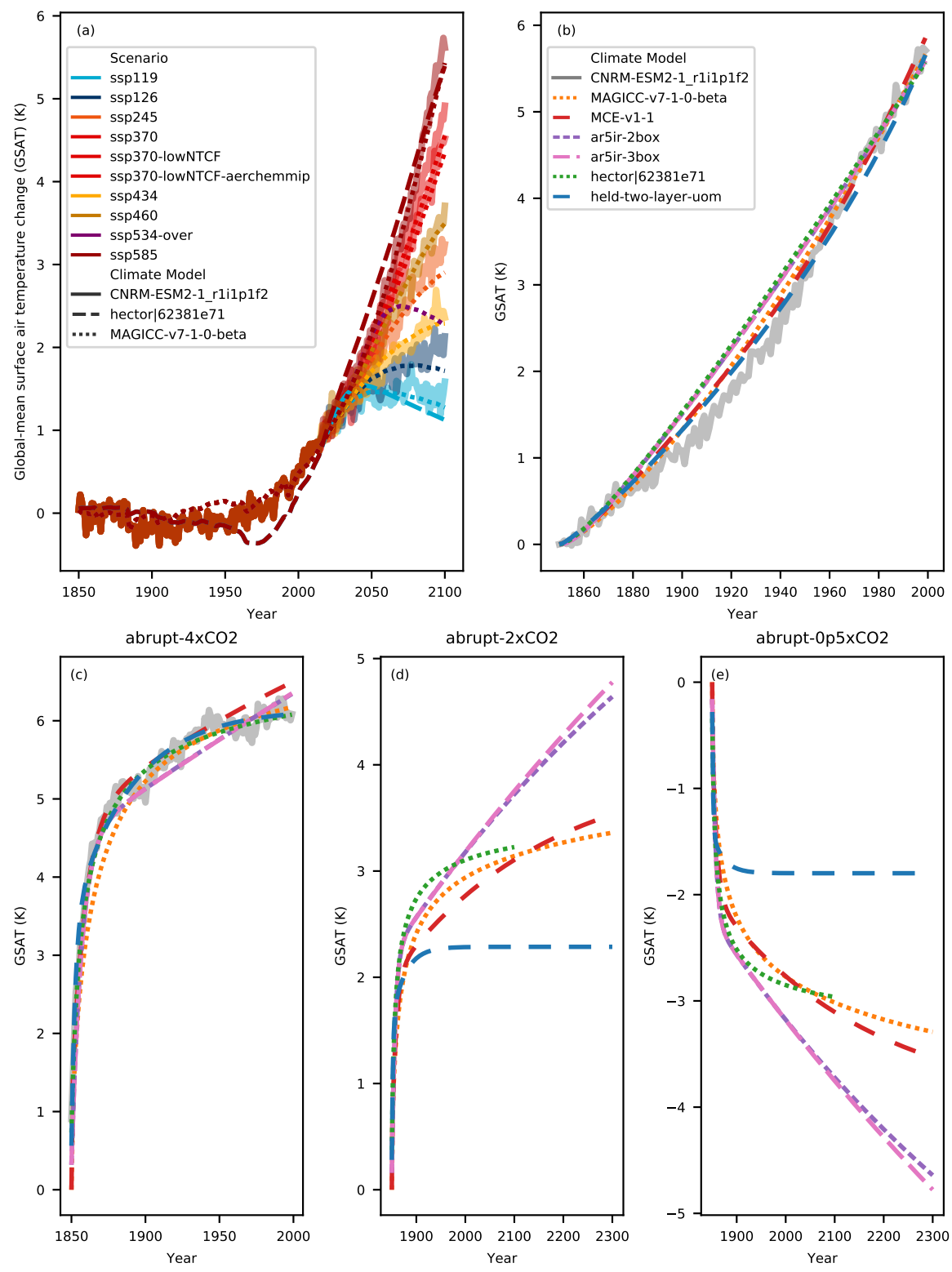

Figure S29. Emulation of CNRM-ESM2-1_r1i1p1f2 by RCMs in RCMIP Phase 1. The thick transparent lines are the target CMIP6 model output (here from CNRM-ESM2-1_r1i1p1f2). The thin lines are emulations from different RCMs. Panel (a) shows results for scenario based experiments while panels (b) - (e) show results for idealised CO2-only experiments (note that panels (b) - (e) share the same legend). 

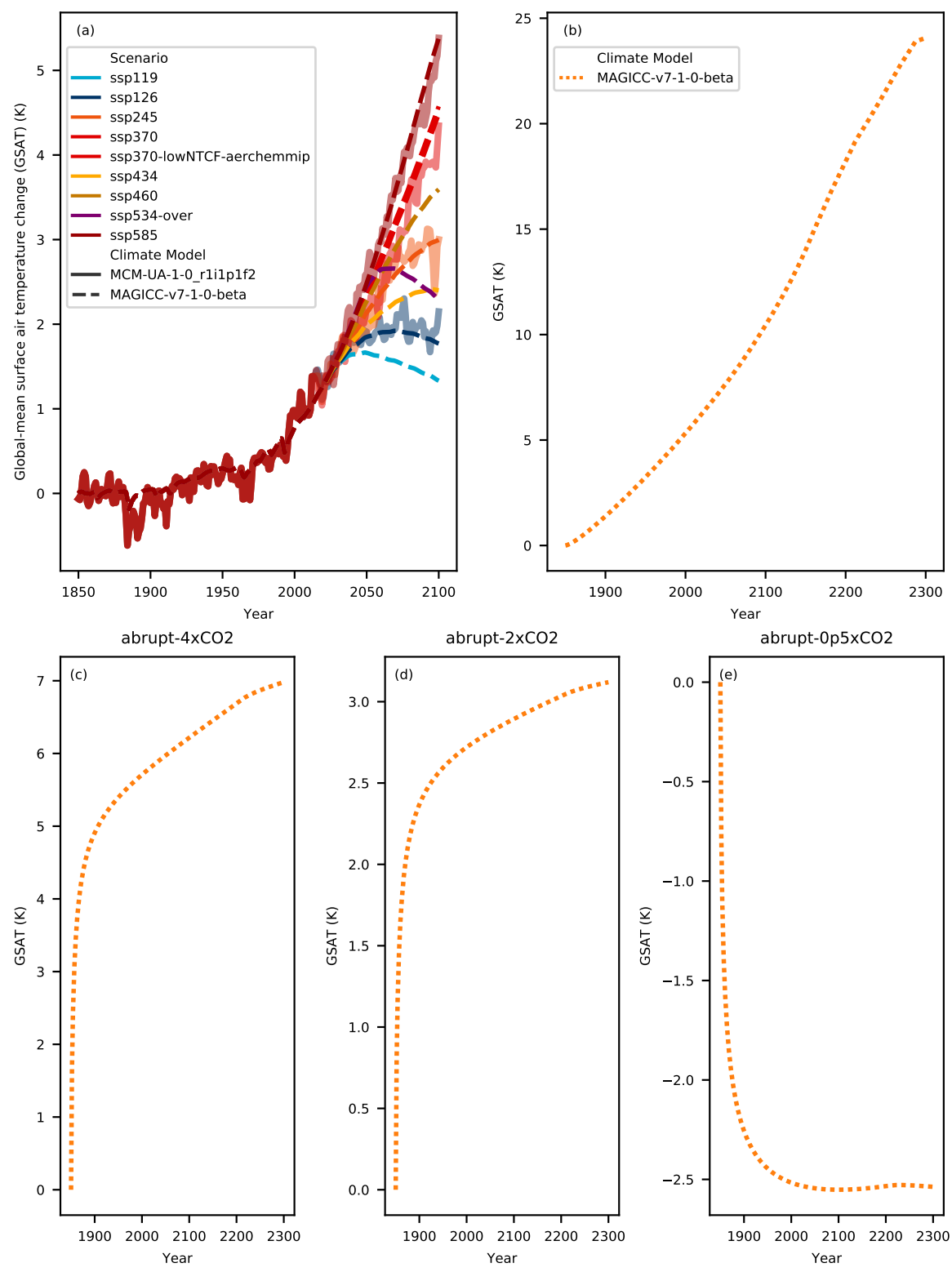

Figure S30. Emulation of MCM-UA-1-0_r1i1p1f2 by RCMs in RCMIP Phase 1. The thick transparent lines are the target CMIP6 model output (here from MCM-UA-1-0_r1ilp1f2). The thin lines are emulations from different RCMs. Panel (a) shows results for scenario based experiments while panels (b) - (e) show results for idealised CO2-only experiments (note that panels (b) - (e) share the same legend). 

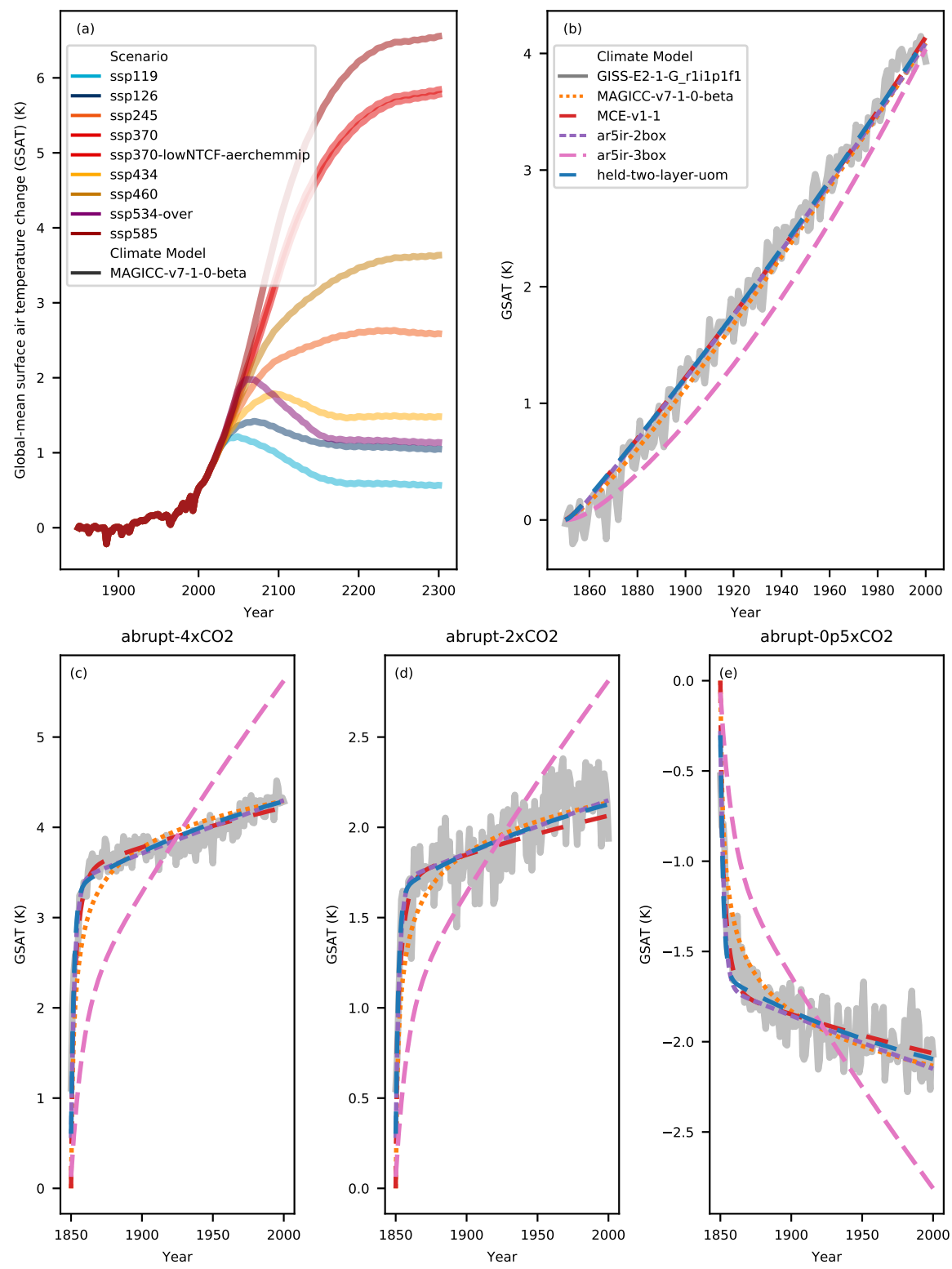

Figure S31. Emulation of GISS-E2-1-G_r1i1p1f1 by RCMs in RCMIP Phase 1. The thick transparent lines are the target CMIP6 model output (here from GISS-E2-1-G_r1i1p1f1). The thin lines are emulations from different RCMs. Panel (a) shows results for scenario based experiments while panels (b) - (e) show results for idealised CO2-only experiments (note that panels (b) - (e) share the same legend). 

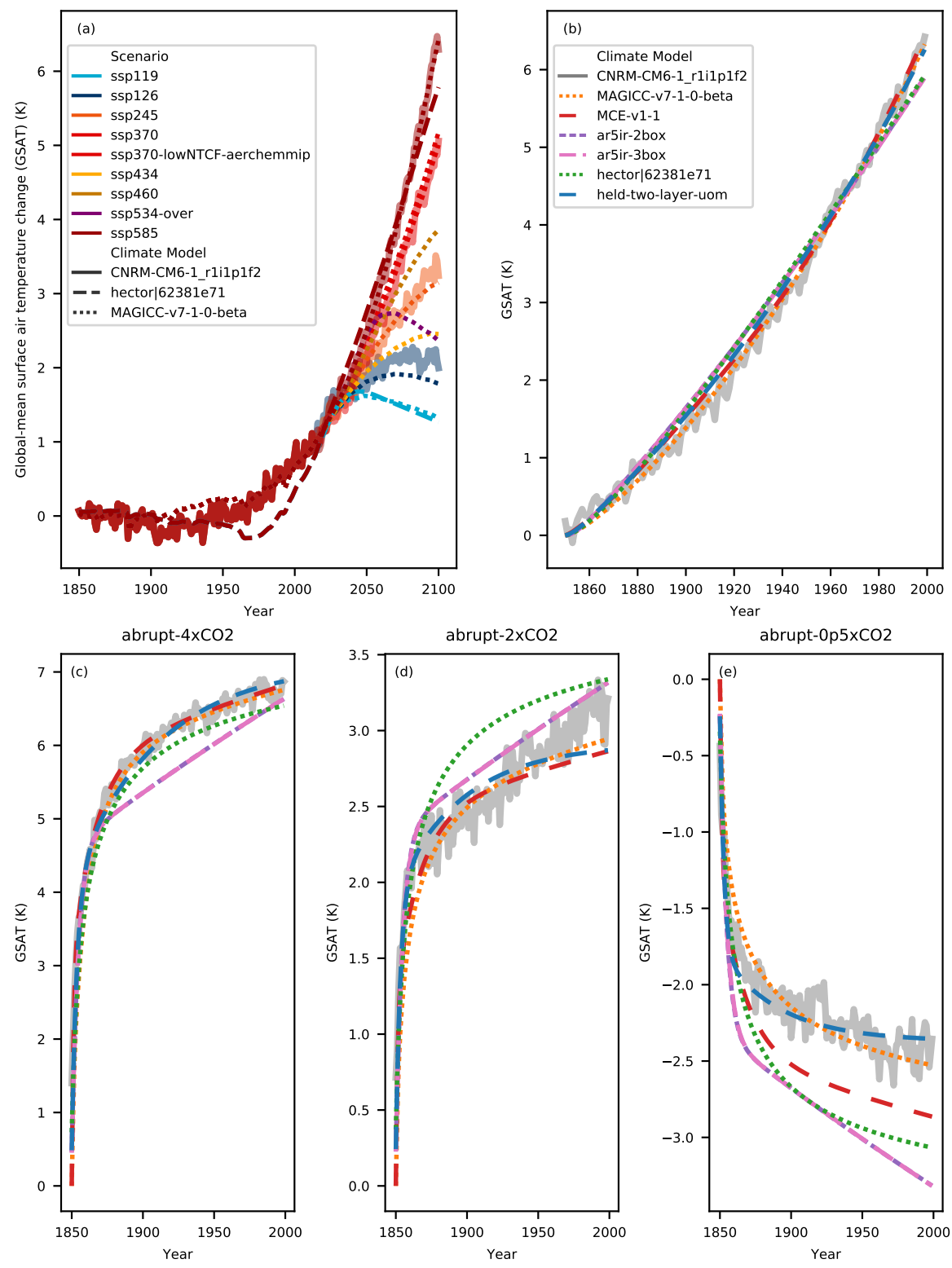

Figure S32. Emulation of CNRM-CM6-1_r1ilp1f2 by RCMs in RCMIP Phase 1. The thick transparent lines are the target CMIP6 model output (here from CNRM-CM6-1_r1i1p1f2). The thin lines are emulations from different RCMs. Panel (a) shows results for scenario based experiments while panels (b) - (e) show results for idealised CO2-only experiments (note that panels (b) - (e) share the same legend). 

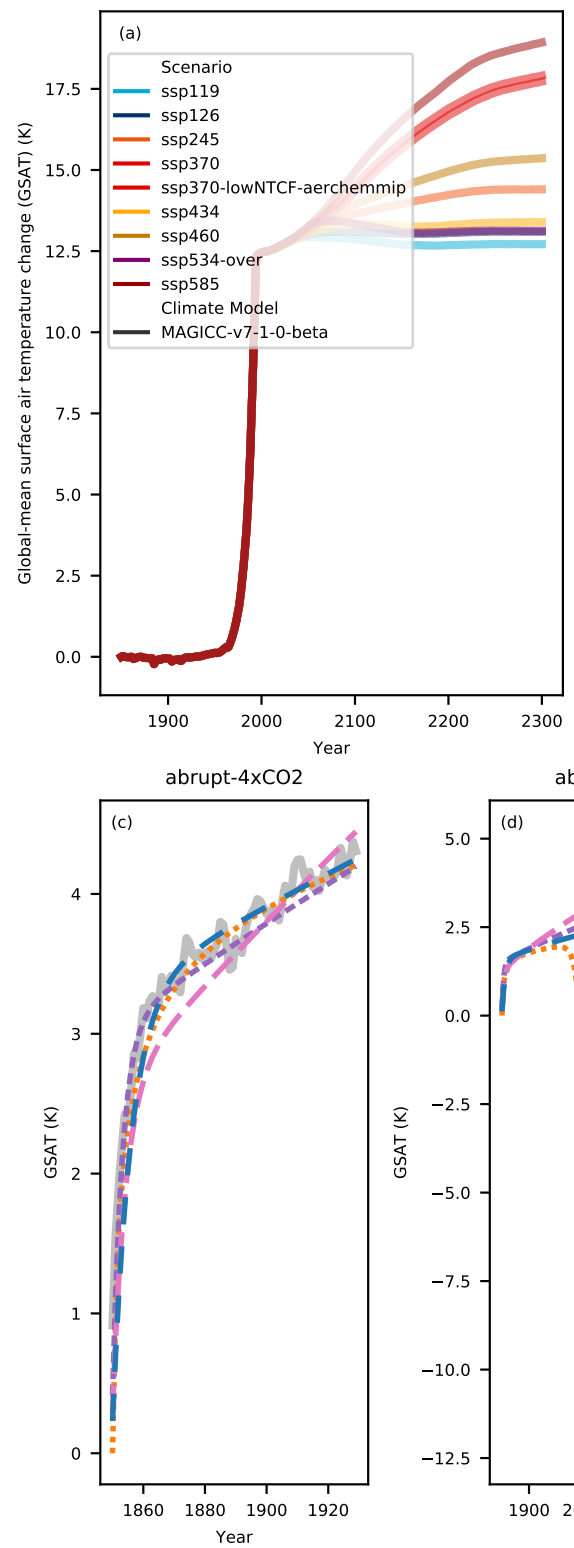

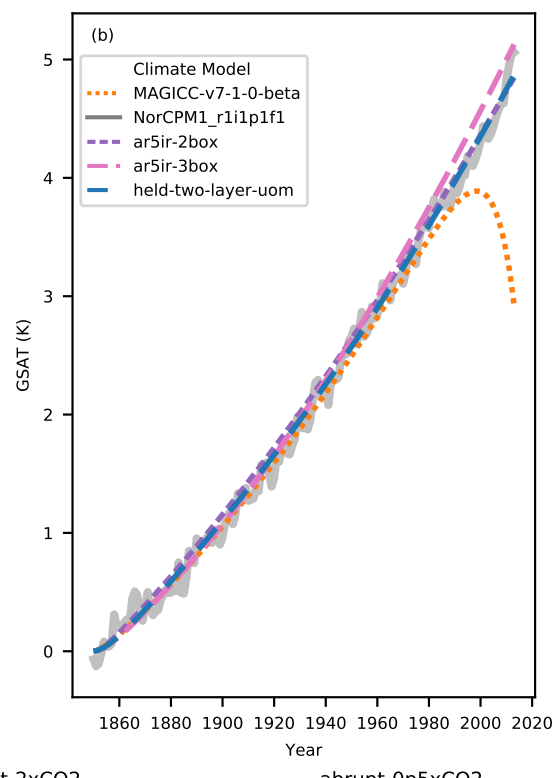

abrupt-0p5xCO2

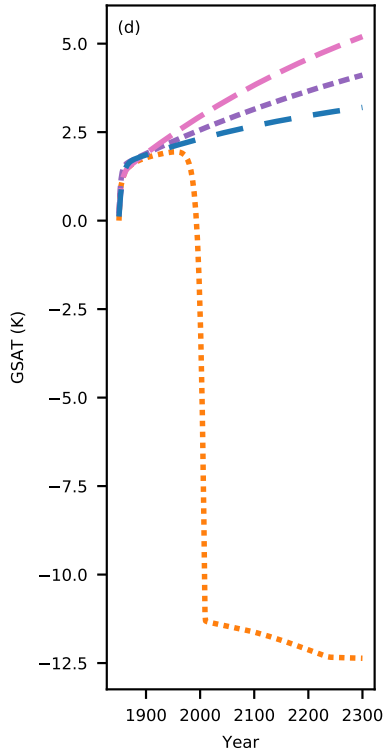

Figure S33. Emulation of NorCPM1_r1i1p1f1 by RCMs in RCMIP Phase 1. The thick transparent lines are the target CMIP6 model output (here from NorCPM1_r1i1p1f1). The thin lines are emulations from different RCMs. Panel (a) shows results for scenario based experiments while panels (b) - (e) show results for idealised CO2-only experiments (note that panels (b) - (e) share the same legend). 
Table S3. RCMIP Phase 1 experiment overview (also available at rcmip.org). In the 'drivers' column, the acronyms show the inputs which are provided to the models in order to perform the run. $\mathrm{CC}$ : $\mathrm{CO}_{2}$ concentrations; $\mathrm{CO}$ : non- $\mathrm{CO}_{2} \mathrm{WMGHG}$ concentrations; $\mathrm{EC}$ : $\mathrm{CO} 2$ emissions; EO: non- $\mathrm{CO}_{2}$ WMGHG emissions; A: aerosol emissions; S: solar effective radiative forcing; V: volcanic effective radiative forcing. ESDOC refers to the Earth System Documentation service (https://search.es-doc.org/).

\begin{tabular}{|c|c|c|c|c|}
\hline ID & Drivers & Summary & Further information & Tier \\
\hline piControl & $\mathrm{CC}, \mathrm{CO}, \mathrm{A}, \mathrm{S}, \mathrm{V}$ & Pre-industrial control simulation. & ESDOC & 1 \\
\hline esm-piControl & $\mathrm{EC}, \mathrm{CO}, \mathrm{A}, \mathrm{S}, \mathrm{V}$ & $\begin{array}{l}\text { Pre-industrial control simulation with zero } \\
\text { anthropogenic perturbation to } \mathrm{CO} 2 \text { emis- } \\
\text { sions. }\end{array}$ & ESDOC & 1 \\
\hline
\end{tabular}

\begin{tabular}{|c|c|c|c|c|}
\hline $\begin{array}{l}\text { esm-piControl- } \\
\text { allGHG }\end{array}$ & $\mathrm{EC}, \mathrm{EO}, \mathrm{A}, \mathrm{S}, \mathrm{V}$ & $\begin{array}{l}\text { Pre-industrial control simulation with zero } \\
\text { anthropogenic perturbation to GHG emis- } \\
\text { sions. }\end{array}$ & RCMIP specific experiment & 2 \\
\hline $1 \mathrm{pctCO} 2$ & $\mathrm{CC}$ & $\begin{array}{l}1 \% \text { per year increase in atmospheric } \mathrm{CO} 2 \\
\text { concentrations. }\end{array}$ & ESDOC & 1 \\
\hline abrupt-4xCO2 & $\mathrm{CC}$ & $\begin{array}{l}\text { Abrupt quadrupling of atmospheric } \mathrm{CO} 2 \\
\text { concentrations. }\end{array}$ & ESDOC & 1 \\
\hline abrupt-2xCO2 & $\mathrm{CC}$ & $\begin{array}{l}\text { Abrupt doubling of atmospheric } \mathrm{CO} 2 \text { con- } \\
\text { centrations. }\end{array}$ & ESDOC & 1 \\
\hline
\end{tabular}


Table S3. Continued.

\begin{tabular}{|c|c|c|c|c|}
\hline ID & Drivers & Summary & Further information & Tier \\
\hline $\begin{array}{l}\text { esm-pi- } \\
\mathrm{CO} 2 \text { pulse }\end{array}$ & EC & $\begin{array}{l}\text { Addition of } 100 \mathrm{GtC} \text { in a single year from } \\
\text { pre-industrial atmosphere, zero } \mathrm{CO} 2 \text { emis- } \\
\text { sions thereafter. }\end{array}$ & ESDOC & 2 \\
\hline $\begin{array}{l}\text { esm-bell- } \\
1000 \mathrm{PgC}\end{array}$ & EC & $\begin{array}{l}\text { Cumulative addition of } 1000 \mathrm{PgC} \text { following } \\
\text { a bell-curved shaped emissions timeseries. }\end{array}$ & ESDOC & 3 \\
\hline $\begin{array}{l}\text { esm-bell- } \\
2000 \mathrm{PgC}\end{array}$ & EC & $\begin{array}{l}\text { Cumulative addition of } 2000 \mathrm{PgC} \text { following } \\
\text { a bell-curved shaped emissions timeseries. }\end{array}$ & ESDOC & 3 \\
\hline $\begin{array}{l}\text { esm-bell- } \\
750 \mathrm{PgC}\end{array}$ & EC & $\begin{array}{l}\text { Cumulative addition of } 750 \mathrm{PgC} \text { following } \\
\text { a bell-curved shaped emissions timeseries. }\end{array}$ & ESDOC & 3 \\
\hline $\begin{array}{l}\text { historical- } \\
\text { cmip5 }\end{array}$ & $\mathrm{CC}, \mathrm{CO}, \mathrm{A}, \mathrm{S}, \mathrm{V}$ & $\begin{array}{l}\text { Simulation of } 1850-2004 \text {, matching forc- } \\
\text { ings as estimated in CMIP5. }\end{array}$ & $\begin{array}{l}\text { http://www.pik-potsdam.de/ } \\
\sim \text { mmalte/rcps/ }\end{array}$ & 2 \\
\hline hist-aer & A & $\begin{array}{l}\text { Simulation of } 1850-2014 \text { with aerosol } \\
\text { emissions only. }\end{array}$ & ESDOC & 3 \\
\hline hist-CO2 & $\mathrm{CC}$ & $\begin{array}{l}\text { Simulation of } 1850-2014 \text { with changing } \\
\text { CO2 concentrations only. }\end{array}$ & ESDOC & 3 \\
\hline hist-volc & $\mathrm{V}$ & $\begin{array}{l}\text { Simulation of } 1850-2014 \text { with changing } \\
\text { volcanic forcing only. }\end{array}$ & ESDOC & 3 \\
\hline ssp119 & $\mathrm{CC}, \mathrm{CO}, \mathrm{A}, \mathrm{S}, \mathrm{V}$ & $\begin{array}{l}\text { Low-end scenario reaching radiative forc- } \\
\text { ing } \sim 1.9 \mathrm{Wm}^{-2} \text { in } 2100 \text { (using the SSP1 } \\
\text { socioeconomic storyline). }\end{array}$ & ESDOC & 1 \\
\hline esm-ssp119 & $\mathrm{EC}, \mathrm{CO}, \mathrm{A}, \mathrm{S}, \mathrm{V}$ & As above except $\mathrm{CO} 2$ emissions driven. & ESDOC & 1 \\
\hline $\begin{array}{l}\text { esm-ssp119- } \\
\text { allGHG }\end{array}$ & $\mathrm{EC}, \mathrm{EO}, \mathrm{A}, \mathrm{S}, \mathrm{V}$ & As above except all GHG emissions driven. & ESDOC & 2 \\
\hline
\end{tabular}


Table S3. Continued.

\begin{tabular}{|c|c|c|c|c|}
\hline ID & Drivers & Summary & Further information & Tier \\
\hline ssp126 & $\mathrm{CC}, \mathrm{CO}, \mathrm{A}, \mathrm{S}, \mathrm{V}$ & $\begin{array}{l}\text { Update of RCP2.6 based on the SSP1 so- } \\
\text { cioeconomic storyline. }\end{array}$ & ESDOC & 2 \\
\hline esm-ssp126 & $\mathrm{EC}, \mathrm{CO}, \mathrm{A}, \mathrm{S}, \mathrm{V}$ & As above except $\mathrm{CO} 2$ emissions driven. & ESDOC & 3 \\
\hline $\operatorname{ssp} 245$ & $\mathrm{CC}, \mathrm{CO}, \mathrm{A}, \mathrm{S}, \mathrm{V}$ & $\begin{array}{l}\text { Update of } \mathrm{RCP} 4.5 \text { based on the SSP2 so- } \\
\text { cioeconomic storyline. }\end{array}$ & ESDOC & 2 \\
\hline esm-ssp245 & $\mathrm{EC}, \mathrm{CO}, \mathrm{A}, \mathrm{S}, \mathrm{V}$ & As above except $\mathrm{CO} 2$ emissions driven. & ESDOC & 3 \\
\hline $\operatorname{ssp} 370$ & $\mathrm{CC}, \mathrm{CO}, \mathrm{A}, \mathrm{S}, \mathrm{V}$ & $\begin{array}{l}\text { Gap-filling scenario reaching radiative forc- } \\
\text { ing } \sim 7.0 \mathrm{Wm}^{-2} \text { in } 2100 \text { (using the SSP3 } \\
\text { socioeconomic storyline). }\end{array}$ & ESDOC & 2 \\
\hline esm-ssp370 & $\mathrm{EC}, \mathrm{CO}, \mathrm{A}, \mathrm{S}, \mathrm{V}$ & As above except $\mathrm{CO} 2$ emissions driven. & ESDOC & 3 \\
\hline $\begin{array}{l}\text { esm-ssp370- } \\
\text { allGHG }\end{array}$ & $\mathrm{EC}, \mathrm{EO}, \mathrm{A}, \mathrm{S}, \mathrm{V}$ & As above except all GHG emissions driven. & ESDOC & 3 \\
\hline $\begin{array}{l}\text { esm-ssp370- } \\
\text { lowNTCF- } \\
\text { allGHG }\end{array}$ & $\mathrm{EC}, \mathrm{EO}, \mathrm{A}, \mathrm{S}, \mathrm{V}$ & As above except all GHG emissions driven. & ESDOC & 3 \\
\hline $\begin{array}{l}\text { ssp370- } \\
\text { lowNTCF- } \\
\text { gidden }\end{array}$ & $\mathrm{CC}, \mathrm{CO}, \mathrm{A}, \mathrm{S}, \mathrm{V}$ & $\begin{array}{l}\text { Comparison scenario, follows the ssp370- } \\
\text { lowNTCF quantification presented in Gid- } \\
\text { den et al. (2019). }\end{array}$ & RCMIP specific & 3 \\
\hline
\end{tabular}


Table S3. Continued.

\begin{tabular}{|c|c|c|c|c|}
\hline ID & Drivers & Summary & Further information & Tier \\
\hline $\begin{array}{l}\text { esm-ssp370- } \\
\text { lowNTCF- } \\
\text { gidden }\end{array}$ & $\mathrm{EC}, \mathrm{CO}, \mathrm{A}, \mathrm{S}, \mathrm{V}$ & As above except $\mathrm{CO} 2$ emissions driven. & RCMIP specific & 3 \\
\hline $\begin{array}{l}\text { esm-ssp370- } \\
\text { lowNTCF- } \\
\text { gidden-allGHG }\end{array}$ & $\mathrm{EC}, \mathrm{EO}, \mathrm{A}, \mathrm{S}, \mathrm{V}$ & As above except all GHG emissions driven. & RCMIP specific & 3 \\
\hline ssp434 & $\mathrm{CC}, \mathrm{CO}, \mathrm{A}, \mathrm{S}, \mathrm{V}$ & $\begin{array}{l}\text { Gap-filling scenario reaching radiative forc- } \\
\text { ing } \sim 3.4 \mathrm{Wm}^{-2} \text { in } 2100 \text { with low near- } \\
\text { term climate forcers (using the SSP } 4 \text { so- } \\
\text { cioeconomic storyline). }\end{array}$ & ESDOC & 2 \\
\hline $\begin{array}{l}\text { esm-ssp434- } \\
\text { allGHG }\end{array}$ & $\mathrm{EC}, \mathrm{EO}, \mathrm{A}, \mathrm{S}, \mathrm{V}$ & As above except all GHG emissions driven. & ESDOC & 3 \\
\hline $\operatorname{ssp} 460$ & $\mathrm{CC}, \mathrm{CO}, \mathrm{A}, \mathrm{S}, \mathrm{V}$ & $\begin{array}{l}\text { Update of RCP6.0 based on the SSP } 4 \text { so- } \\
\text { cioeconomic storyline. }\end{array}$ & ESDOC & 2 \\
\hline esm-ssp460 & $\mathrm{EC}, \mathrm{CO}, \mathrm{A}, \mathrm{S}, \mathrm{V}$ & As above except $\mathrm{CO} 2$ emissions driven. & ESDOC & 3 \\
\hline $\begin{array}{l}\text { esm-ssp460- } \\
\text { allGHG }\end{array}$ & $\mathrm{EC}, \mathrm{EO}, \mathrm{A}, \mathrm{S}, \mathrm{V}$ & As above except all GHG emissions driven. & ESDOC & 3 \\
\hline $\begin{array}{l}\text { esm-ssp534- } \\
\text { over }\end{array}$ & $\mathrm{EC}, \mathrm{CO}, \mathrm{A}, \mathrm{S}, \mathrm{V}$ & As above except $\mathrm{CO} 2$ emissions driven. & ESDOC & 3 \\
\hline $\begin{array}{l}\text { esm-ssp534- } \\
\text { over-allGHG }\end{array}$ & $\mathrm{EC}, \mathrm{EO}, \mathrm{A}, \mathrm{S}, \mathrm{V}$ & As above except all GHG emissions driven. & ESDOC & 3 \\
\hline ssp585 & $\mathrm{CC}, \mathrm{CO}, \mathrm{A}, \mathrm{S}, \mathrm{V}$ & $\begin{array}{l}\text { Update of RCP } 8.5 \text { based on the SSP } 5 \text { so- } \\
\text { cioeconomic storyline. }\end{array}$ & ESDOC & 1 \\
\hline esm-ssp585 & $\mathrm{EC}, \mathrm{CO}, \mathrm{A}, \mathrm{S}, \mathrm{V}$ & As above except $\mathrm{CO} 2$ emissions driven. & ESDOC & 1 \\
\hline
\end{tabular}


Table S3. Continued.

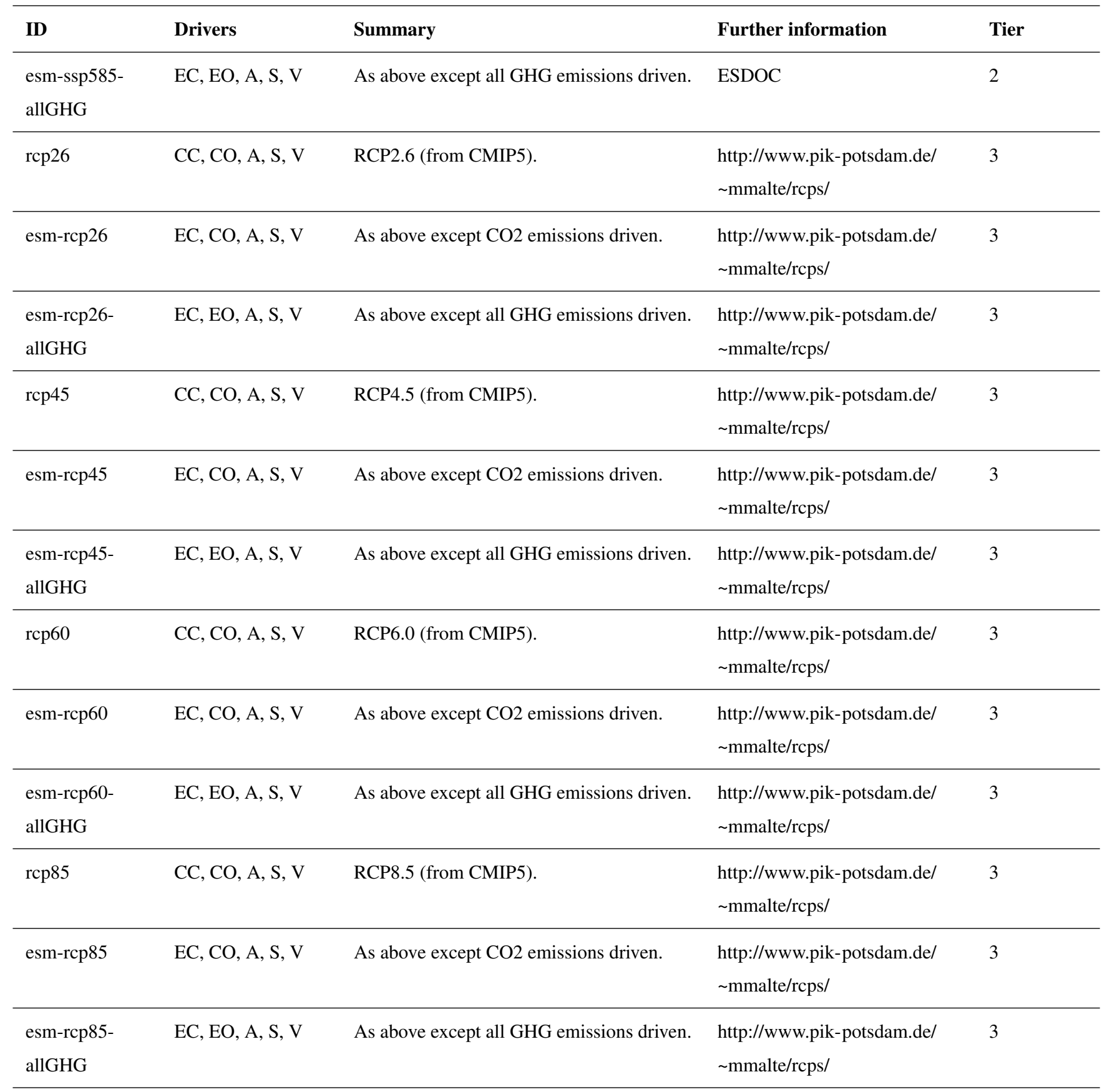


Table S4. RCMIP Phase 1 variable overview (also available at rcmip.org).

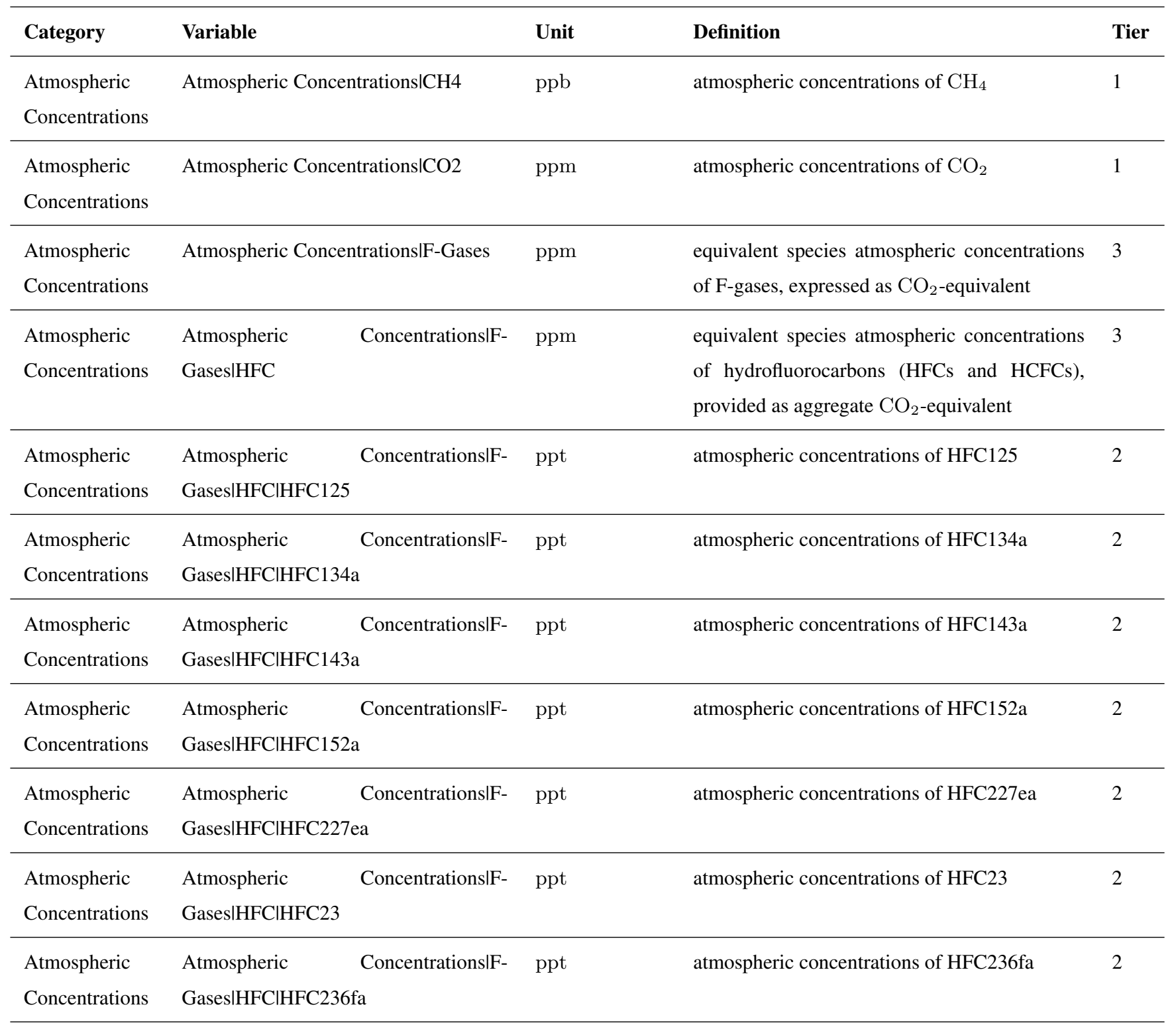


Table S4. Continued.

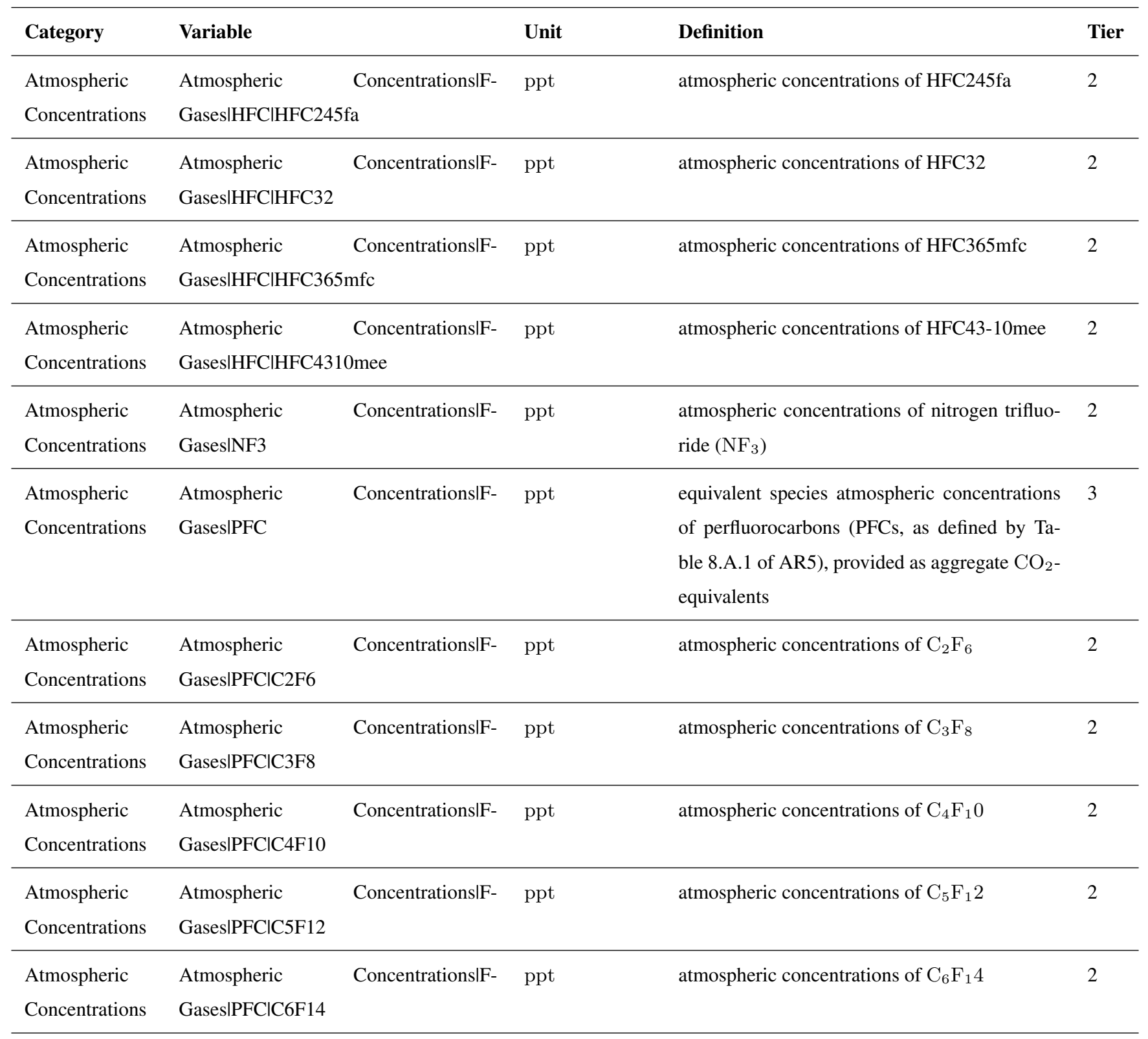


Table S4. Continued.

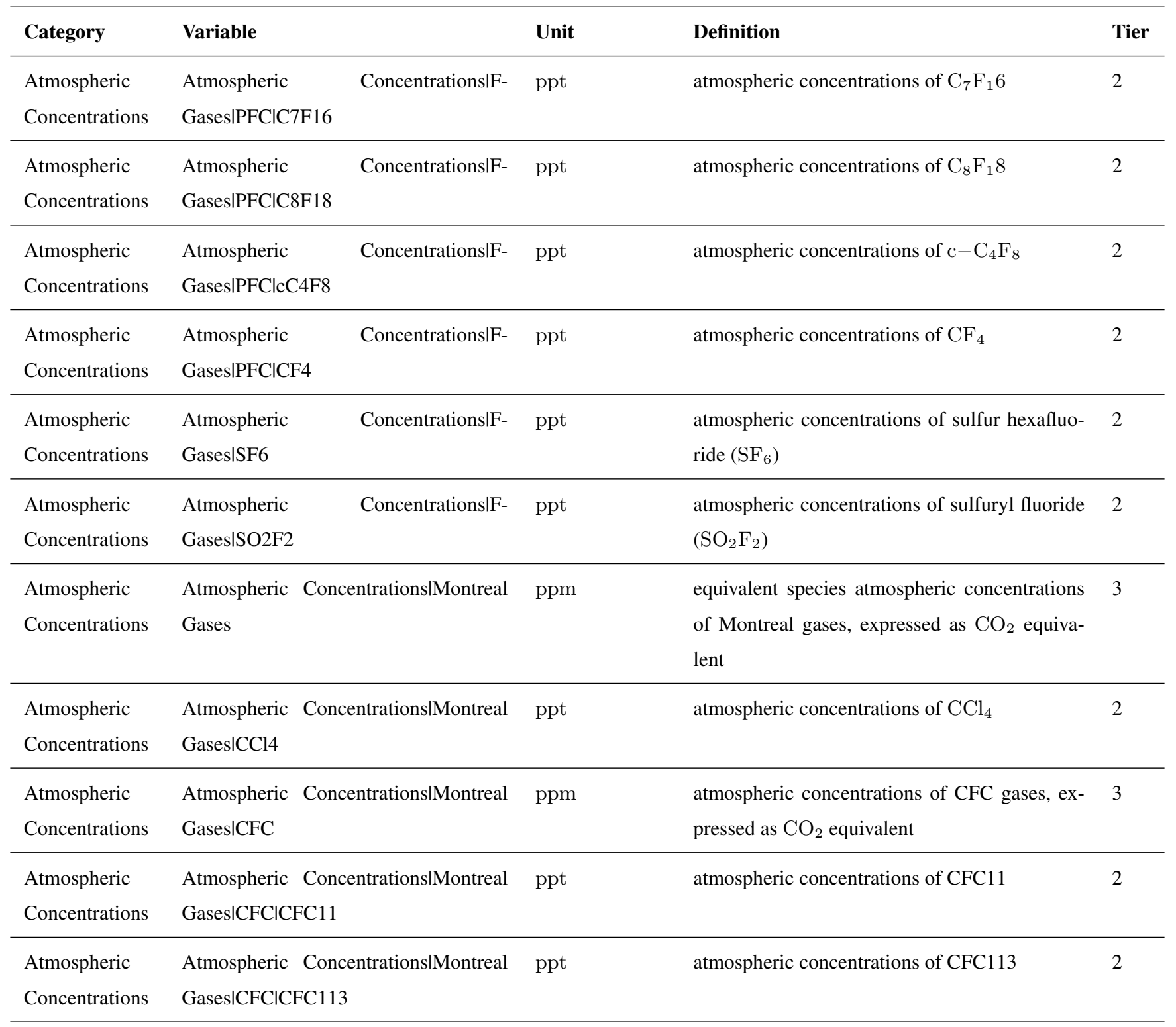


Table S4. Continued.

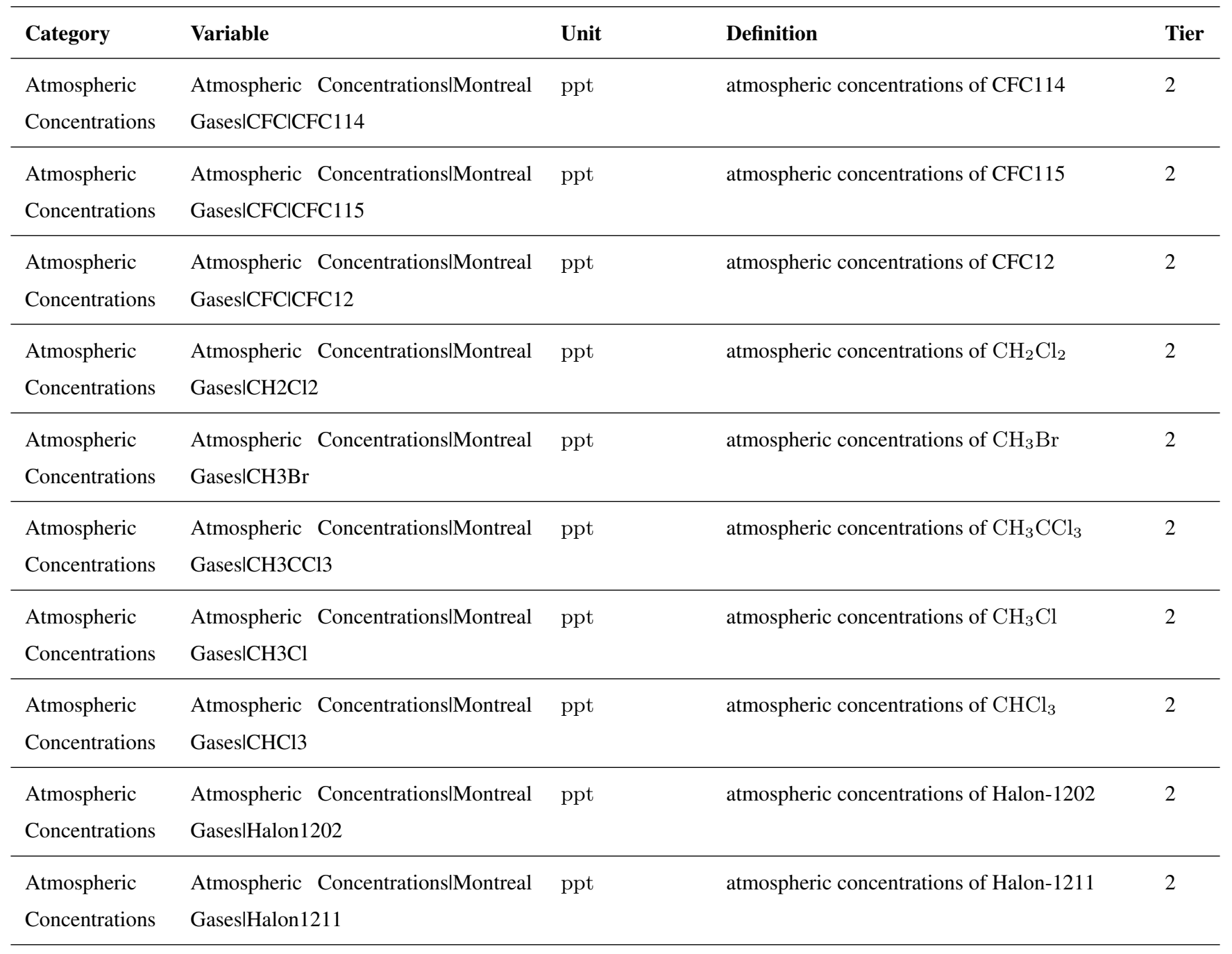


Table S4. Continued.

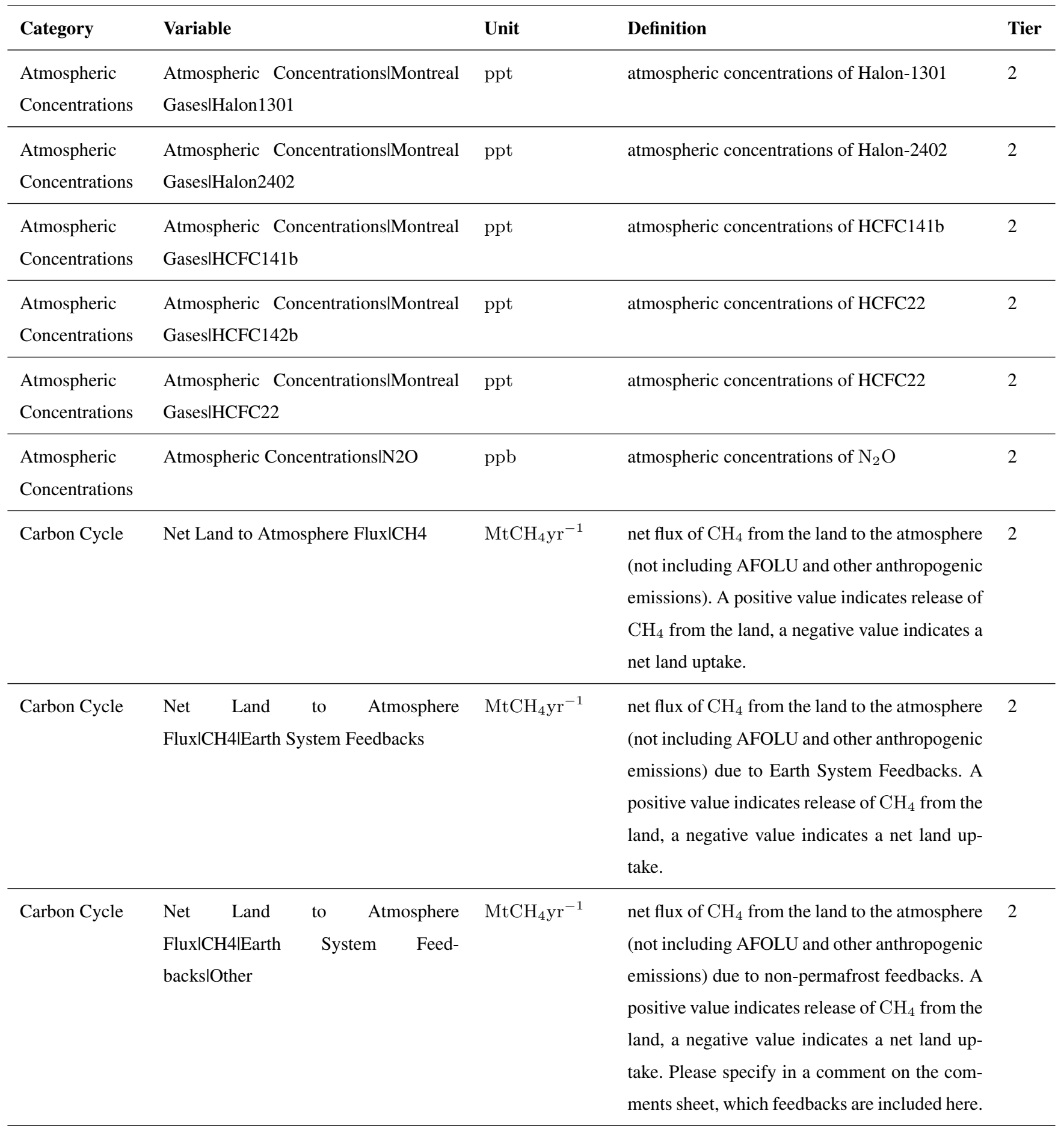


Table S4. Continued.

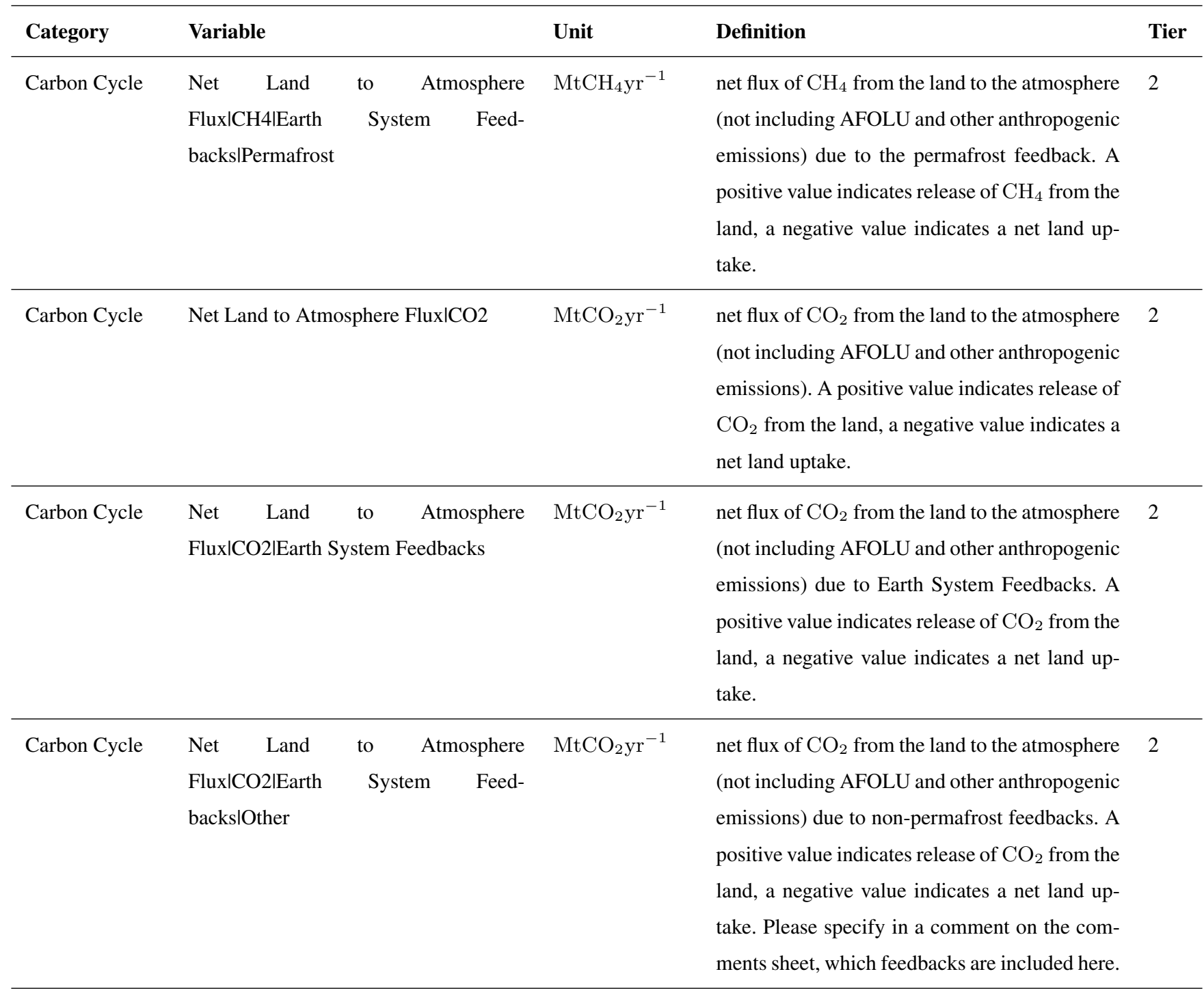


Table S4. Continued.

\begin{tabular}{|c|c|c|c|c|}
\hline Category & Variable & Unit & Definition & Tier \\
\hline Carbon Cycle & $\begin{array}{l}\text { Net Land to Atmosphere } \\
\text { Flux|CO2|Earth } \\
\text { backs|Permafrost }\end{array}$ & $\mathrm{MtCO}_{2} \mathrm{yr}^{-1}$ & $\begin{array}{l}\text { net flux of } \mathrm{CO}_{2} \text { from the land to the atmosphere } \\
\text { (not including } \mathrm{AFOLU} \text { and other anthropogenic } \\
\text { emissions) due to the permafrost feedback. A } \\
\text { positive value indicates release of } \mathrm{CO}_{2} \text { from the } \\
\text { land, a negative value indicates a net land up- } \\
\text { take. }\end{array}$ & 2 \\
\hline Carbon Cycle & Net Ocean to Atmosphere Flux|CH4 & $\mathrm{MtCH}_{4} \mathrm{yr}^{-1}$ & $\begin{array}{l}\text { net flux of } \mathrm{CH}_{4} \text { from the ocean to the at- } \\
\text { mosphere (not including anthropogenic emis- } \\
\text { sions). A positive value indicates release of } \\
\mathrm{CH}_{4} \text { from the ocean, a negative value indicates } \\
\text { a net ocean uptake. }\end{array}$ & 2 \\
\hline Carbon Cycle & Net Ocean to Atmosphere Flux|CO2 & $\mathrm{MtCO}_{2 \mathrm{yr}^{-1}}$ & $\begin{array}{l}\text { cumulative net flux of } \mathrm{CO}_{2} \text { from the ocean } \\
\text { to the atmosphere (not including anthropogenic } \\
\text { emissions). A positive value indicates release of } \\
\mathrm{CO}_{2} \text { from the ocean, a negative value indicates } \\
\text { a net ocean uptake. }\end{array}$ & 2 \\
\hline Carbon Cycle & $\begin{array}{l}\text { Cumulative Net Land to Atmosphere } \\
\text { Flux } \mid \mathrm{CH} 4\end{array}$ & $\mathrm{MtCH}_{4}$ & $\begin{array}{l}\text { cumulative net flux of } \mathrm{CH}_{4} \text { from the land to the } \\
\text { atmosphere (not including AFOLU and other } \\
\text { anthropogenic emissions). A positive value in- } \\
\text { dicates release of } \mathrm{CH}_{4} \text { from the land, a negative } \\
\text { value indicates a net land uptake. }\end{array}$ & 2 \\
\hline
\end{tabular}


Table S4. Continued.

\begin{tabular}{|c|c|c|c|c|}
\hline Category & Variable & Unit & Definition & Tier \\
\hline Carbon Cycle & $\begin{array}{l}\text { Cumulative Net Land to Atmo- } \\
\text { sphere Flux|CH4|Earth System Feed- } \\
\text { backs|Other }\end{array}$ & $\mathrm{MtCH}_{4}$ & $\begin{array}{l}\text { cumulative net flux of } \mathrm{CH}_{4} \text { from the land } \\
\text { to the atmosphere (not including AFOLU and } \\
\text { other anthropogenic emissions) due to non- } \\
\text { permafrost feedbacks. A positive value indi- } \\
\text { cates release of } \mathrm{CH}_{4} \text { from the land, a negative } \\
\text { value indicates a net land uptake. Please spec- } \\
\text { ify in a comment on the comments sheet, which } \\
\text { feedbacks are included here. }\end{array}$ & 2 \\
\hline Carbon Cycle & $\begin{array}{l}\text { Cumulative Net Land to Atmo- } \\
\text { sphere Flux|CH4|Earth System Feed- } \\
\text { backs|Permafrost }\end{array}$ & $\mathrm{MtCH}_{4}$ & $\begin{array}{l}\text { cumulative net flux of } \mathrm{CH}_{4} \text { from the land to the } \\
\text { atmosphere (not including AFOLU and other } \\
\text { anthropogenic emissions) due to the permafrost } \\
\text { feedback. A positive value indicates release of } \\
\mathrm{CH}_{4} \text { from the land, a negative value indicates a } \\
\text { net land uptake. }\end{array}$ & 2 \\
\hline Carbon Cycle & $\begin{array}{l}\text { Cumulative Net Land to Atmosphere } \\
\text { Flux|CO2 }\end{array}$ & $\mathrm{MtCO}_{2}$ & $\begin{array}{l}\text { cumulative net flux of } \mathrm{CO}_{2} \text { from the land to the } \\
\text { atmosphere (not including AFOLU and other } \\
\text { anthropogenic emissions). A positive value in- } \\
\text { dicates release of } \mathrm{CO}_{2} \text { from the land, a negative } \\
\text { value indicates a net land uptake. }\end{array}$ & 2 \\
\hline
\end{tabular}


Table S4. Continued.

\begin{tabular}{|c|c|c|c|c|}
\hline Category & Variable & Unit & Definition & Tier \\
\hline Carbon Cycle & $\begin{array}{l}\text { Cumulative Net Land to Atmo- } \\
\text { sphere Flux|CO2|Earth System Feed- } \\
\text { backs|Other }\end{array}$ & $\mathrm{MtCO}_{2}$ & $\begin{array}{l}\text { cumulative net flux of } \mathrm{CO}_{2} \text { from the land } \\
\text { to the atmosphere (not including AFOLU and } \\
\text { other anthropogenic emissions) due to non- } \\
\text { permafrost feedbacks. A positive value indi- } \\
\text { cates release of } \mathrm{CO}_{2} \text { from the land, a negative } \\
\text { value indicates a net land uptake. Please spec- } \\
\text { ify in a comment on the comments sheet, which } \\
\text { feedbacks are included here. }\end{array}$ & 2 \\
\hline Carbon Cycle & $\begin{array}{l}\text { Cumulative Net Land to Atmo- } \\
\text { sphere Flux|CO2|Earth System Feed- } \\
\text { backs|Permafrost }\end{array}$ & $\mathrm{MtCO}_{2}$ & $\begin{array}{l}\text { cumulative net flux of } \mathrm{CO}_{2} \text { from the land to the } \\
\text { atmosphere (not including AFOLU and other } \\
\text { anthropogenic emissions) due to the permafrost } \\
\text { feedback. A positive value indicates release of } \\
\mathrm{CO}_{2} \text { from the land, a negative value indicates a } \\
\text { net land uptake. }\end{array}$ & 2 \\
\hline Carbon Cycle & $\begin{array}{l}\text { Cumulative Net Ocean to Atmosphere } \\
\text { FluxICH4 }\end{array}$ & $\mathrm{MtCH}_{4}$ & $\begin{array}{l}\text { cumulative net flux of } \mathrm{CH}_{4} \text { from the ocean } \\
\text { to the atmosphere (not including anthropogenic } \\
\text { emissions). A positive value indicates release of } \\
\mathrm{CH}_{4} \text { from the ocean, a negative value indicates } \\
\text { a net ocean uptake. }\end{array}$ & 2 \\
\hline
\end{tabular}


Table S4. Continued.

\begin{tabular}{|c|c|c|c|c|}
\hline Category & Variable & Unit & Definition & Tier \\
\hline Carbon Cycle & Carbon PoollAtmosphere & $\mathrm{MtCO}_{2}$ & $\begin{array}{l}\text { total amount of } \mathrm{CO}_{2} \text { in the atmospheric carbon } \\
\text { pool }\end{array}$ & 2 \\
\hline Carbon Cycle & Carbon PoollSoil & $\mathrm{MtCO}_{2}$ & total amount of $\mathrm{CO}_{2}$ in the soil carbon pool & 2 \\
\hline Carbon Cycle & Carbon PoollPlant & $\mathrm{MtCO}_{2}$ & total amount of $\mathrm{CO}_{2}$ in the plant carbon pool & 2 \\
\hline Carbon Cycle & Net Primary Productivity & $\mathrm{MtCO}_{2} \mathrm{yr}^{-1}$ & global total net primary productivity & 2 \\
\hline $\mathrm{CCS}$ & Carbon Sequestration & $\mathrm{MtCO}_{2 \mathrm{yr}^{-1}}$ & $\begin{array}{l}\text { total carbon dioxide emissions captured and } \\
\text { stored }\end{array}$ & 1 \\
\hline CCS & Carbon Sequestration|CCSIBiomass & $\mathrm{MtCO}_{2 \mathrm{yr}^{-1}}$ & $\begin{array}{l}\text { total carbon dioxide emissions captured from } \\
\text { bioenergy use and stored in geological deposits } \\
\text { (e.g. in depleted oil and gas fields, unmined } \\
\text { coal seams, saline aquifers) and the deep ocean, } \\
\text { stored amounts should be reported as positive } \\
\text { numbers }\end{array}$ & 2 \\
\hline
\end{tabular}


Table S4. Continued.

\begin{tabular}{|c|c|c|c|c|}
\hline Category & Variable & Unit & Definition & Tier \\
\hline CCS & $\begin{array}{l}\text { Carbon SequestrationlDirect Air Cap- } \\
\text { ture }\end{array}$ & $\mathrm{MtCO}_{2} \mathrm{yr}^{-1}$ & $\begin{array}{l}\text { total carbon dioxide sequestered through direct } \\
\text { air capture }\end{array}$ & 2 \\
\hline $\mathrm{CCS}$ & $\begin{array}{l}\text { Carbon Sequestration|Enhanced Weath- } \\
\text { ering }\end{array}$ & $\mathrm{MtCO}_{2} \mathrm{yr}^{-1}$ & $\begin{array}{l}\text { total carbon dioxide sequestered through en- } \\
\text { hanced weathering }\end{array}$ & 2 \\
\hline $\mathrm{CCS}$ & Carbon SequestrationlLand Use & $\mathrm{MtCO}_{2} \mathrm{yr}^{-1}$ & $\begin{array}{l}\text { total carbon dioxide sequestered through land- } \\
\text { based sinks (e.g., afforestation, soil carbon en- } \\
\text { hancement, biochar) }\end{array}$ & 2 \\
\hline CCS & $\begin{array}{l}\text { Carbon } \quad \text { SequestrationlLand } \\
\text { UselBiochar }\end{array}$ & $\mathrm{MtCO}_{2} \mathrm{yr}^{-1}$ & $\begin{array}{l}\text { total carbon dioxide sequestered through } \\
\text { biochar }\end{array}$ & 2 \\
\hline $\mathrm{CCS}$ & Carbon SequestrationlLand UselOther & $\mathrm{MtCO}_{2} \mathrm{yr}^{-1}$ & $\begin{array}{l}\text { total carbon dioxide sequestered through other } \\
\text { land-based mitigation techniques }\end{array}$ & 2 \\
\hline $\mathrm{CCS}$ & $\begin{array}{l}\text { Carbon SequestrationlLand UselSoil } \\
\text { Carbon Management }\end{array}$ & $\mathrm{MtCO}_{2} \mathrm{yr}^{-1}$ & $\begin{array}{l}\text { total carbon dioxide sequestered through soil } \\
\text { carbon management techniques }\end{array}$ & 2 \\
\hline Climate & Effective Climate Sensitivity & $\mathrm{K}$ & $\begin{array}{l}\text { effective climate sensitivity over time, here de- } \\
\text { fined as ECS_eff }(t)=\text { Delta } T(t) * R F 2 x /(R F(t) \\
\text { - } d H / d t) \text { where ECS_eff is effective climate } \\
\text { sensitivity, Delta } T(t) \text { is Surface Air Tempera- } \\
\text { ture Change, } \mathrm{RF} 2 \mathrm{x} \text { is radiative forcing due to } \\
\text { a doubling of atmospheric } \mathrm{CO}_{2} \text { concentrations, } \\
\mathrm{RF}(\mathrm{t}) \text { is radiative forcing and } \mathrm{dH} / \mathrm{dt} \text { is the en- } \\
\text { ergy imbalance at the top of the atmosphere } \\
\text { (likely equal to ocean heat uptake in most of our } \\
\text { reduced complexity models) }\end{array}$ & 2 \\
\hline
\end{tabular}


Table S4. Continued.

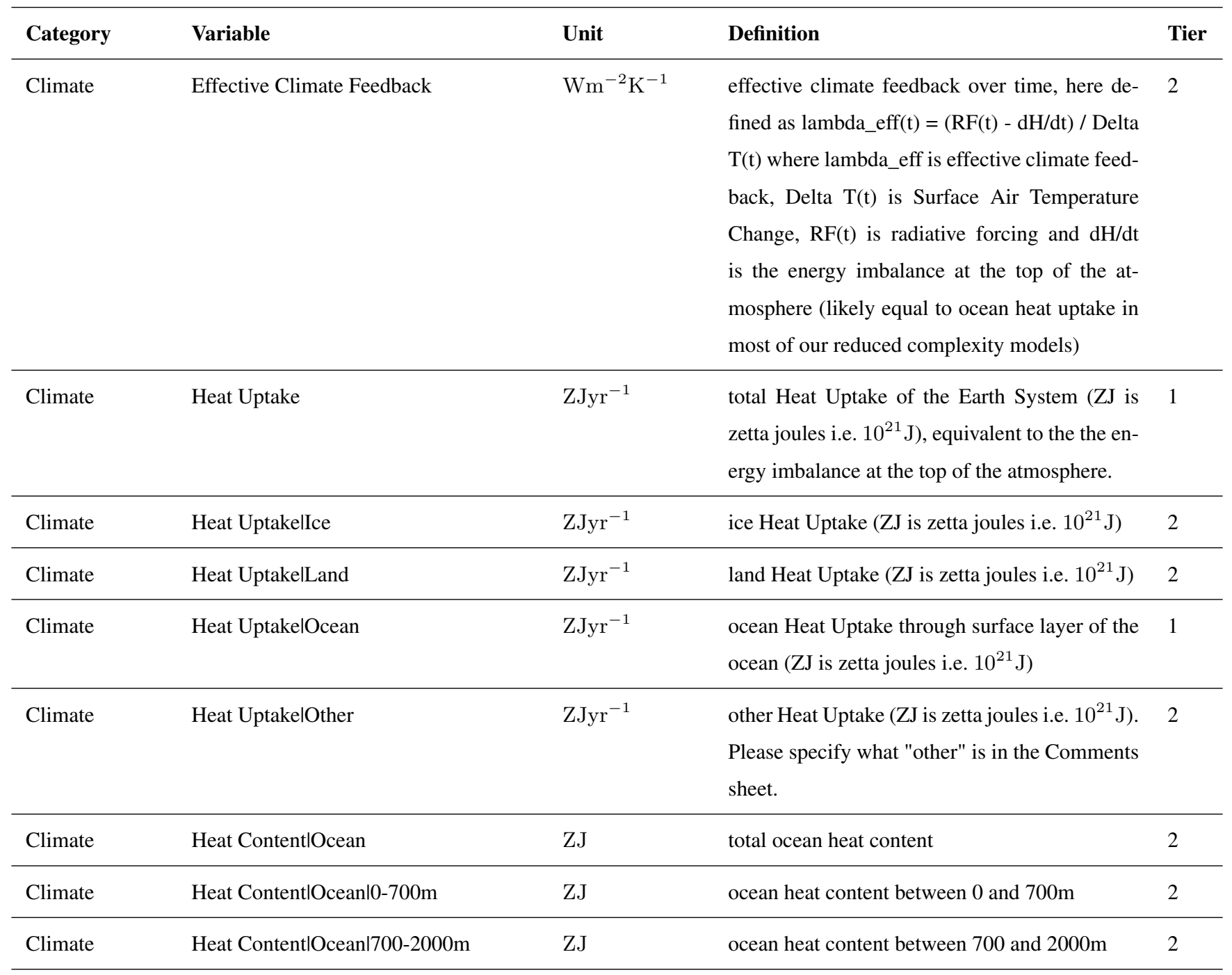


Table S4. Continued.

\begin{tabular}{|c|c|c|c|c|}
\hline Category & Variable & Unit & Definition & Tier \\
\hline Climate & Instantaneous TCRE & $\mathrm{K} / \mathrm{MtCO}_{2}$ & $\begin{array}{l}\text { warming per unit cumulative } \mathrm{CO}_{2} \text { (this } \\
\text { should simply be your 'Surface Air Tem- } \\
\text { perature Change' divided by 'Cumulative } \\
\text { Emissions } \mid \mathrm{CO}_{2} \text { ') }\end{array}$ & 2 \\
\hline Climate & Surface Ocean Temperature Change & $\mathrm{K}$ & $\begin{array}{l}\text { change in surface layer ocean tempertaure. } \\
\text { Please note reference period in comment sheet. }\end{array}$ & 1 \\
\hline $\begin{array}{l}\text { Cumulative } \\
\text { Emissions }\end{array}$ & Cumulative Emissions $\mid \mathrm{CO} 2$ & $\mathrm{MtCO}_{2}$ & cumulative carbon dioxide emissions & 1 \\
\hline $\begin{array}{l}\text { Cumulative } \\
\text { Emissions }\end{array}$ & $\begin{array}{l}\text { Cumulative Emissions|CO2/MAGICC } \\
\text { AFOLU }\end{array}$ & $\mathrm{MtCO}_{2}$ & $\begin{array}{l}\text { cumulative carbon dioxide emissions from agri- } \\
\text { culture, forestry and other land use (IPCC cat- } \\
\text { egory } 3 \text { ), excluding any fossil-fuel based emis- } \\
\text { sions in the Agricultural sector (hence not iden- } \\
\text { tical to WG3 AFOLU) }\end{array}$ & 2 \\
\hline $\begin{array}{l}\text { Cumulative } \\
\text { Emissions }\end{array}$ & Cumulative Emissions|CO2|Other & $\mathrm{MtCO}_{2}$ & $\begin{array}{l}\text { cumulative carbon dioxide emissions from } \\
\text { other sources (please provide a definition of } \\
\text { other sources in this category in the 'comments' } \\
\text { tab) }\end{array}$ & 2 \\
\hline
\end{tabular}


Table S4. Continued.

\begin{tabular}{|c|c|c|c|c|}
\hline Category & Variable & Unit & Definition & Tier \\
\hline $\begin{array}{l}\text { Effective Ra- } \\
\text { diative Forcing }\end{array}$ & Effective Radiative Forcing & $\mathrm{Wm}^{-2}$ & $\begin{array}{l}\text { effective radiative forcing from all anthro- } \\
\text { pogenic and natural sources (after stratospheric } \\
\text { temperature adjustments and rapid adjustments) }\end{array}$ & 1 \\
\hline $\begin{array}{l}\text { Effective Ra- } \\
\text { diative Forcing }\end{array}$ & $\begin{array}{l}\text { Effective Radiative } \\
\text { inglAnthropogenic }\end{array}$ & $\mathrm{Wm}^{-2}$ & $\begin{array}{l}\text { effective radiative forcing from all anthro- } \\
\text { pogenic sources (after stratospheric tempera- } \\
\text { ture adjustments and rapid adjustments) }\end{array}$ & 1 \\
\hline $\begin{array}{l}\text { Effective Ra- } \\
\text { diative Forcing }\end{array}$ & $\begin{array}{l}\text { Effective Radiative } \\
\text { inglAnthropogeniclAerosols }\end{array}$ & $\mathrm{Wm}^{-2}$ & $\begin{array}{l}\text { effective radiative forcing from aerosols (after } \\
\text { stratospheric temperature adjustments and rapid } \\
\text { adjustments) }\end{array}$ & 1 \\
\hline $\begin{array}{l}\text { Effective Ra- } \\
\text { diative Forcing }\end{array}$ & $\begin{array}{l}\text { Effective Radiative } \\
\text { Forcing|Anthropogenic|Aerosols|Aerosols- } \\
\text { radiation Interactions }\end{array}$ & $\mathrm{Wm}^{-2}$ & $\begin{array}{l}\text { effective radiative forcing from aerosol- } \\
\text { radiative effects (after stratospheric tempera- } \\
\text { ture adjustments and rapid adjustments), note } \\
\text { that the breakdown of this variable can come in } \\
\text { multiple different forms }\end{array}$ & 2 \\
\hline $\begin{array}{l}\text { Effective Ra- } \\
\text { diative Forcing }\end{array}$ & $\begin{array}{l}\text { Effective Radiative } \\
\text { Forcing|Anthropogenic|Aerosols|Aerosols- } \\
\text { radiation Interactions|BC and OC|BC }\end{array}$ & $\mathrm{Wm}^{-2}$ & $\begin{array}{l}\text { effective radiative forcing from aerosol- } \\
\text { radiative effects from black carbon emissions } \\
\text { (after stratospheric temperature adjustments } \\
\text { and rapid adjustments) }\end{array}$ & 2 \\
\hline
\end{tabular}


Table S4. Continued.

\begin{tabular}{|c|c|c|c|c|}
\hline Category & Variable & Unit & Definition & Tier \\
\hline $\begin{array}{l}\text { Effective Ra- } \\
\text { diative Forcing }\end{array}$ & $\begin{array}{l}\text { Effective Radiative } \\
\text { Forcing|Anthropogenic|Aerosols|Aerosols- } \\
\text { radiation Interactions|BC and } \\
\text { OC|BC|Fossil and Industrial }\end{array}$ & $\mathrm{Wm}^{-2}$ & $\begin{array}{l}\text { effective radiative forcing from aerosol- } \\
\text { radiative effects from black carbon fossil } \\
\text { and industrial emissions (after stratospheric } \\
\text { temperature adjustments and rapid adjustments) }\end{array}$ & 2 \\
\hline $\begin{array}{l}\text { Effective Ra- } \\
\text { diative Forcing }\end{array}$ & $\begin{array}{l}\text { Effective Radiative } \\
\text { Forcing|Anthropogenic|Aerosols|Aerosols- } \\
\text { radiation Interactions|BC and } \\
\text { OClOC|Fossil and Industrial }\end{array}$ & $\mathrm{Wm}^{-2}$ & $\begin{array}{l}\text { effective radiative forcing from aerosol- } \\
\text { radiative effects from organic carbon fossil } \\
\text { and industrial emissions (after stratospheric } \\
\text { temperature adjustments and rapid adjustments) }\end{array}$ & 2 \\
\hline $\begin{array}{l}\text { Effective Ra- } \\
\text { diative Forcing }\end{array}$ & $\begin{array}{l}\text { Effective Radiative } \\
\text { Forcing|Anthropogenic|Aerosols|Aerosols- } \\
\text { radiation Interactions|Biomass Burning }\end{array}$ & $\mathrm{Wm}^{-2}$ & $\begin{array}{l}\text { effective radiative forcing from aerosol- } \\
\text { radiative effects from biomass burning } \\
\text { emissions (after stratospheric temperature } \\
\text { adjustments and rapid adjustments) }\end{array}$ & 2 \\
\hline
\end{tabular}


Table S4. Continued.

\begin{tabular}{|c|c|c|c|c|}
\hline Category & Variable & Unit & Definition & Tier \\
\hline $\begin{array}{l}\text { Effective Ra- } \\
\text { diative Forcing }\end{array}$ & $\begin{array}{l}\text { Effective Radiative } \\
\text { Forcing|Anthropogenic|Aerosols|Aerosols- } \\
\text { radiation Interactions|Biomass Burn- } \\
\text { ing|BC and OC }\end{array}$ & $\mathrm{Wm}^{-2}$ & $\begin{array}{l}\text { effective radiative forcing from aerosol- } \\
\text { radiative effects from black and organic carbon } \\
\text { biomass burning emissions (after stratospheric } \\
\text { temperature adjustments and rapid adjustments) }\end{array}$ & 2 \\
\hline $\begin{array}{l}\text { Effective Ra- } \\
\text { diative Forcing }\end{array}$ & $\begin{array}{l}\text { Effective } \\
\text { Forcing|Anthropogenic|Aerosols|Aerosols- } \\
\text { radiation Interactions|Biomass Burn- } \\
\text { inglNH3 }\end{array}$ & $\mathrm{Wm}^{-2}$ & $\begin{array}{l}\text { effective radiative forcing from aerosol- } \\
\text { radiative effects from ammonia biomass } \\
\text { burning emissions (after stratospheric tempera- } \\
\text { ture adjustments and rapid adjustments) }\end{array}$ & 2 \\
\hline $\begin{array}{l}\text { Effective Ra- } \\
\text { diative Forcing }\end{array}$ & $\begin{array}{l}\text { Effective Radiative } \\
\text { Forcing|Anthropogenic|Aerosols|Aerosols- } \\
\text { radiation Interactions|Biomass Burn- } \\
\text { inglNitrate }\end{array}$ & $\mathrm{Wm}^{-2}$ & $\begin{array}{l}\text { effective radiative forcing from aerosol- } \\
\text { radiative effects from nitrate precursor biomass } \\
\text { burning emissions (after stratospheric tempera- } \\
\text { ture adjustments and rapid adjustments) }\end{array}$ & 2 \\
\hline
\end{tabular}


Table S4. Continued.

\begin{tabular}{|c|c|c|c|c|}
\hline Category & Variable & Unit & Definition & Tier \\
\hline $\begin{array}{l}\text { Effective Ra- } \\
\text { diative Forcing }\end{array}$ & $\begin{array}{l}\text { Effective Radiative } \\
\text { Forcing|Anthropogenic|Aerosols|Aerosols- } \\
\text { radiation Interactions|Biomass Burn- } \\
\text { inglSulfate }\end{array}$ & $\mathrm{Wm}^{-2}$ & $\begin{array}{l}\text { effective radiative forcing from aerosol- } \\
\text { radiative effects from sulfate precursor biomass } \\
\text { burning emissions (after stratospheric tempera- } \\
\text { ture adjustments and rapid adjustments) }\end{array}$ & 2 \\
\hline $\begin{array}{l}\text { Effective Ra- } \\
\text { diative Forcing }\end{array}$ & $\begin{array}{l}\text { Effective Radiative } \\
\text { Forcing|Anthropogenic|Aerosols|Aerosols- } \\
\text { radiation Interactions|Fossil and } \\
\text { Industrial|BC and OCIBC }\end{array}$ & $\mathrm{Wm}^{-2}$ & $\begin{array}{l}\text { effective radiative forcing from aerosol- } \\
\text { radiative effects from black carbon fossil } \\
\text { and industrial emissions (after stratospheric } \\
\text { temperature adjustments and rapid adjustments) }\end{array}$ & 2 \\
\hline $\begin{array}{l}\text { Effective Ra- } \\
\text { diative Forcing }\end{array}$ & $\begin{array}{l}\text { Effective Radiative } \\
\text { Forcing|Anthropogenic|Aerosols|Aerosols- } \\
\text { radiation Interactions|Fossil and } \\
\text { Industrial|BC and OClOC }\end{array}$ & $\mathrm{Wm}^{-2}$ & $\begin{array}{l}\text { effective radiative forcing from aerosol- } \\
\text { radiative effects from organic carbon fossil } \\
\text { and industrial emissions (after stratospheric } \\
\text { temperature adjustments and rapid adjustments) }\end{array}$ & 2 \\
\hline
\end{tabular}


Table S4. Continued.

\begin{tabular}{|c|c|c|c|c|}
\hline Category & Variable & Unit & Definition & Tier \\
\hline $\begin{array}{l}\text { Effective Ra- } \\
\text { diative Forcing }\end{array}$ & $\begin{array}{l}\text { Effective Radiative } \\
\text { Forcing|Anthropogenic|Aerosols|Aerosols- } \\
\text { radiation Interactions|Fossil and } \\
\text { Industrial|NH3 }\end{array}$ & $\mathrm{Wm}^{-2}$ & $\begin{array}{l}\text { effective radiative forcing from aerosol- } \\
\text { radiative effects from ammonia fossil and } \\
\text { industrial emissions (after stratospheric tem- } \\
\text { perature adjustments and rapid adjustments) }\end{array}$ & 2 \\
\hline $\begin{array}{l}\text { Effective Ra- } \\
\text { diative Forcing }\end{array}$ & $\begin{array}{l}\text { Effective Radiative } \\
\text { Forcing|Anthropogenic|Aerosols|Aerosols- } \\
\text { radiation Interactions|Mineral Dust }\end{array}$ & $\mathrm{Wm}^{-2}$ & $\begin{array}{l}\text { effective radiative forcing from aerosol- } \\
\text { radiative effects from mineral dust emissions } \\
\text { (after stratospheric temperature adjustments } \\
\text { and rapid adjustments) }\end{array}$ & 2 \\
\hline $\begin{array}{l}\text { Effective Ra- } \\
\text { diative Forcing }\end{array}$ & $\begin{array}{l}\text { Effective } \quad \text { Radiative } \\
\text { Forcing|Anthropogenic|Aerosols|Aerosols- } \\
\text { radiation Interactions|NH3 }\end{array}$ & $\mathrm{Wm}^{-2}$ & $\begin{array}{l}\text { effective radiative forcing from aerosol- } \\
\text { radiative effects from ammonia emissions } \\
\text { (after stratospheric temperature adjustments } \\
\text { and rapid adjustments) }\end{array}$ & 2 \\
\hline
\end{tabular}


Table S4. Continued.

\begin{tabular}{|c|c|c|c|c|}
\hline Category & Variable & Unit & Definition & Tier \\
\hline $\begin{array}{l}\text { Effective Ra- } \\
\text { diative Forcing }\end{array}$ & $\begin{array}{l}\text { Effective } \quad \text { Radiative } \\
\text { Forcing|Anthropogenic|Aerosols|Aerosols- } \\
\text { radiation Interactions|NH3|Biomass } \\
\text { Burning }\end{array}$ & $\mathrm{Wm}^{-2}$ & $\begin{array}{l}\text { effective radiative forcing from aerosol- } \\
\text { radiative effects from ammonia biomass } \\
\text { burning emissions (after stratospheric tempera- } \\
\text { ture adjustments and rapid adjustments) }\end{array}$ & 2 \\
\hline $\begin{array}{l}\text { Effective Ra- } \\
\text { diative Forcing }\end{array}$ & $\begin{array}{l}\text { Effective } \\
\text { Forcing|Anthropogenic|Aerosols|Aerosols- } \\
\text { radiation Interactions|Nitrate|Biomass } \\
\text { Burning }\end{array}$ & $\mathrm{Wm}^{-2}$ & $\begin{array}{l}\text { effective radiative forcing from aerosol- } \\
\text { radiative effects from nitrate precursor biomass } \\
\text { burning emissions (after stratospheric tempera- } \\
\text { ture adjustments and rapid adjustments) }\end{array}$ & 2 \\
\hline $\begin{array}{l}\text { Effective Ra- } \\
\text { diative Forcing }\end{array}$ & $\begin{array}{l}\text { Effective Radiative } \\
\text { Forcing|Anthropogenic|Aerosols|Aerosols- } \\
\text { radiation Interactions|Nitrate|Fossil and } \\
\text { Industrial }\end{array}$ & $\mathrm{Wm}^{-2}$ & $\begin{array}{l}\text { effective radiative forcing from aerosol- } \\
\text { radiative effects from nitrate fossil and indus- } \\
\text { trial emissions (after stratospheric temperature } \\
\text { adjustments and rapid adjustments) }\end{array}$ & 2 \\
\hline
\end{tabular}


Table S4. Continued.

\begin{tabular}{|c|c|c|c|c|}
\hline Category & Variable & Unit & Definition & Tier \\
\hline $\begin{array}{l}\text { Effective Ra- } \\
\text { diative Forcing }\end{array}$ & $\begin{array}{l}\text { Effective Radiative } \\
\text { Forcing|Anthropogenic|Aerosols|Aerosols- } \\
\text { radiation Interactions|Other }\end{array}$ & $\mathrm{Wm}^{-2}$ & $\begin{array}{l}\text { effective radiative forcing from aerosol- } \\
\text { radiative effects not covered in the other } \\
\text { categories (after stratospheric temperature } \\
\text { adjustments and rapid adjustments) (please } \\
\text { specify in comments) }\end{array}$ & 2 \\
\hline $\begin{array}{l}\text { Effective Ra- } \\
\text { diative Forcing }\end{array}$ & $\begin{array}{l}\text { Effective Radiative } \\
\text { Forcing|Anthropogenic|Aerosols|Aerosols- } \\
\text { radiation Interactions|Sulfate }\end{array}$ & $\mathrm{Wm}^{-2}$ & $\begin{array}{l}\text { effective radiative forcing from aerosol- } \\
\text { radiative effects from sulfate precursor } \\
\text { emissions (after stratospheric temperature } \\
\text { adjustments and rapid adjustments) }\end{array}$ & 2 \\
\hline $\begin{array}{l}\text { Effective Ra- } \\
\text { diative Forcing }\end{array}$ & $\begin{array}{l}\text { Effective Radiative } \\
\text { Forcing|Anthropogenic|Aerosols|Aerosols- } \\
\text { radiation Interactions|Sulfate|Fossil and } \\
\text { Industrial }\end{array}$ & $\mathrm{Wm}^{-2}$ & $\begin{array}{l}\text { effective radiative forcing from aerosol- } \\
\text { radiative effects from sulfate precursor fossil } \\
\text { and industrial emissions (after stratospheric } \\
\text { temperature adjustments and rapid adjustments) }\end{array}$ & 2 \\
\hline $\begin{array}{l}\text { Effective Ra- } \\
\text { diative Forcing }\end{array}$ & $\begin{array}{l}\text { Effective Radiative Forc- } \\
\text { inglAnthropogeniclAlbedo Change }\end{array}$ & $\mathrm{Wm}^{-2}$ & $\begin{array}{l}\text { effective radiative forcing from albedo change } \\
\text { (after stratospheric temperature adjustments } \\
\text { and rapid adjustments) }\end{array}$ & 2 \\
\hline
\end{tabular}


Table S4. Continued.

\begin{tabular}{|c|c|c|c|c|c|}
\hline Category & Variable & & Unit & Definition & Tier \\
\hline $\begin{array}{l}\text { Effective Ra- } \\
\text { diative Forcing }\end{array}$ & $\begin{array}{l}\text { Effective Radiative } \\
\text { inglAnthropogenic|CH4 }\end{array}$ & Forc- & $\mathrm{Wm}^{-2}$ & $\begin{array}{l}\text { effective radiative forcing (after stratospheric } \\
\text { temperature adjustments and rapid adjustments) } \\
\text { of } \mathrm{CH}_{4}\end{array}$ & 2 \\
\hline $\begin{array}{l}\text { Effective Ra- } \\
\text { diative Forcing }\end{array}$ & $\begin{array}{l}\text { Effective Radiative } \\
\text { inglAnthropogenic|CO2 }\end{array}$ & Forc- & $\mathrm{Wm}^{-2}$ & $\begin{array}{l}\text { effective radiative forcing (after stratospheric } \\
\text { temperature adjustments and rapid adjustments) } \\
\text { of } \mathrm{CO}_{2}\end{array}$ & 1 \\
\hline $\begin{array}{l}\text { Effective Ra- } \\
\text { diative Forcing }\end{array}$ & $\begin{array}{l}\text { Effective } \\
\text { Forcing|Anthropogenic|F-G }\end{array}$ & $\begin{array}{l}\text { Radiative } \\
\text { ses }\end{array}$ & $\mathrm{Wm}^{-2}$ & $\begin{array}{l}\text { effective radiative forcing (after stratospheric } \\
\text { temperature adjustments and rapid adjustments) } \\
\text { of F-gases }\end{array}$ & 2 \\
\hline $\begin{array}{l}\text { Effective Ra- } \\
\text { diative Forcing }\end{array}$ & $\begin{array}{l}\text { Effective } \\
\text { Forcing|Anthropogenic|F- } \\
\text { Gases|HFCIHFC125 }\end{array}$ & Radiative & $\mathrm{Wm}^{-2}$ & $\begin{array}{l}\text { effective radiative forcing (after stratospheric } \\
\text { temperature adjustments and rapid adjustments) } \\
\text { of HFC } 125\end{array}$ & 2 \\
\hline $\begin{array}{l}\text { Effective Ra- } \\
\text { diative Forcing }\end{array}$ & $\begin{array}{l}\text { Effective } \\
\text { Forcing|Anthropogenic|F- } \\
\text { Gases|HFC|HFC134a }\end{array}$ & Radiative & $\mathrm{Wm}^{-2}$ & $\begin{array}{l}\text { effective radiative forcing (after stratospheric } \\
\text { temperature adjustments and rapid adjustments) } \\
\text { of HFC134a }\end{array}$ & 2 \\
\hline $\begin{array}{l}\text { Effective Ra- } \\
\text { diative Forcing }\end{array}$ & $\begin{array}{l}\text { Effective } \\
\text { Forcing|Anthropogenic|F- } \\
\text { Gases|HFCIHFC152a }\end{array}$ & Radiative & $\mathrm{Wm}^{-2}$ & $\begin{array}{l}\text { effective radiative forcing (after stratospheric } \\
\text { temperature adjustments and rapid adjustments) } \\
\text { of HFC } 152 \mathrm{a}\end{array}$ & 2 \\
\hline $\begin{array}{l}\text { Effective Ra- } \\
\text { diative Forcing }\end{array}$ & $\begin{array}{l}\text { Effective } \\
\text { Forcing|Anthropogenic|F- } \\
\text { Gases|HFCIHFC227ea }\end{array}$ & Radiative & $\mathrm{Wm}^{-2}$ & $\begin{array}{l}\text { effective radiative forcing (after stratospheric } \\
\text { temperature adjustments and rapid adjustments) } \\
\text { of } \mathrm{HFC} 227 \mathrm{ea}\end{array}$ & 2 \\
\hline
\end{tabular}


Table S4. Continued.

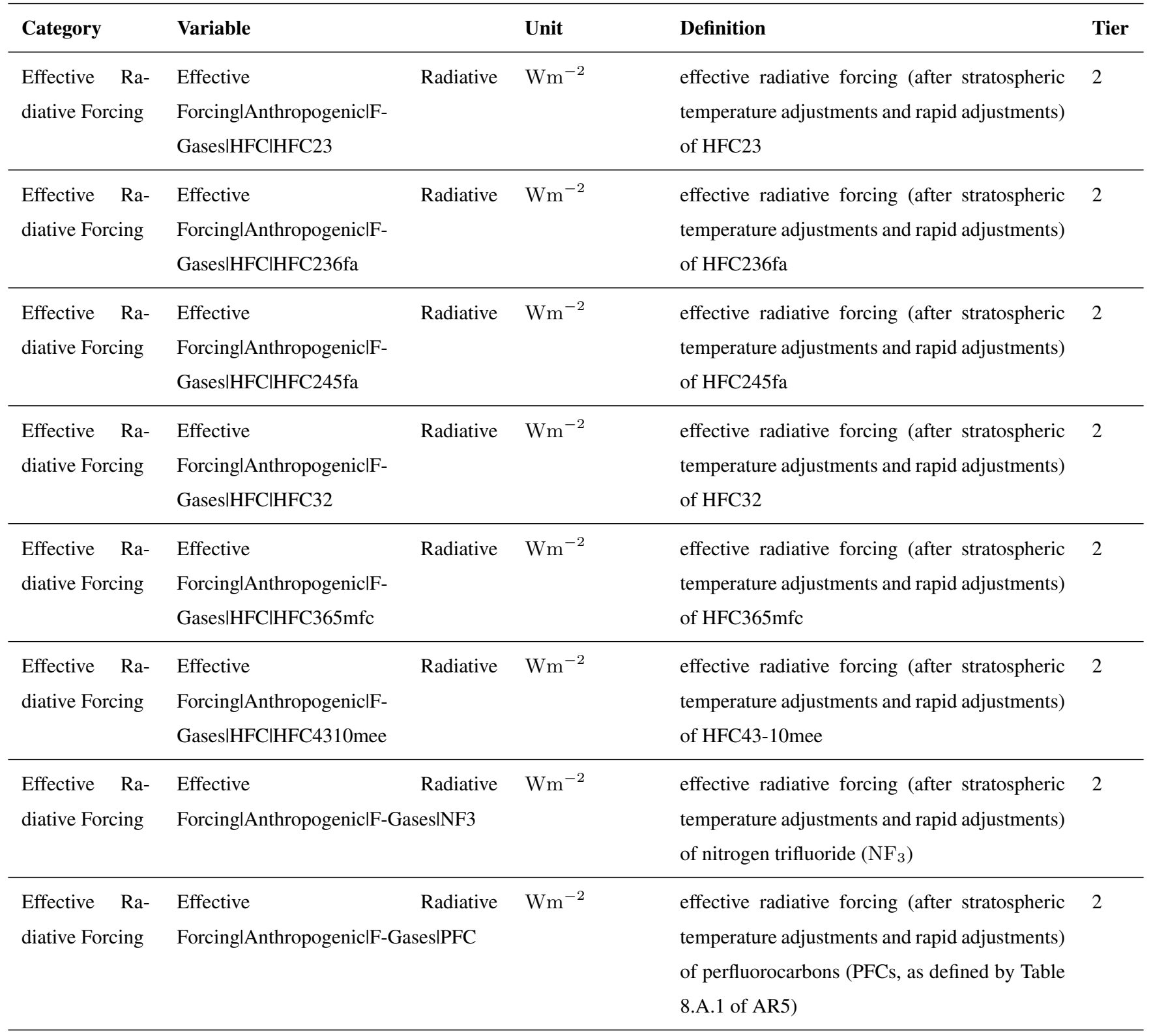


Table S4. Continued.

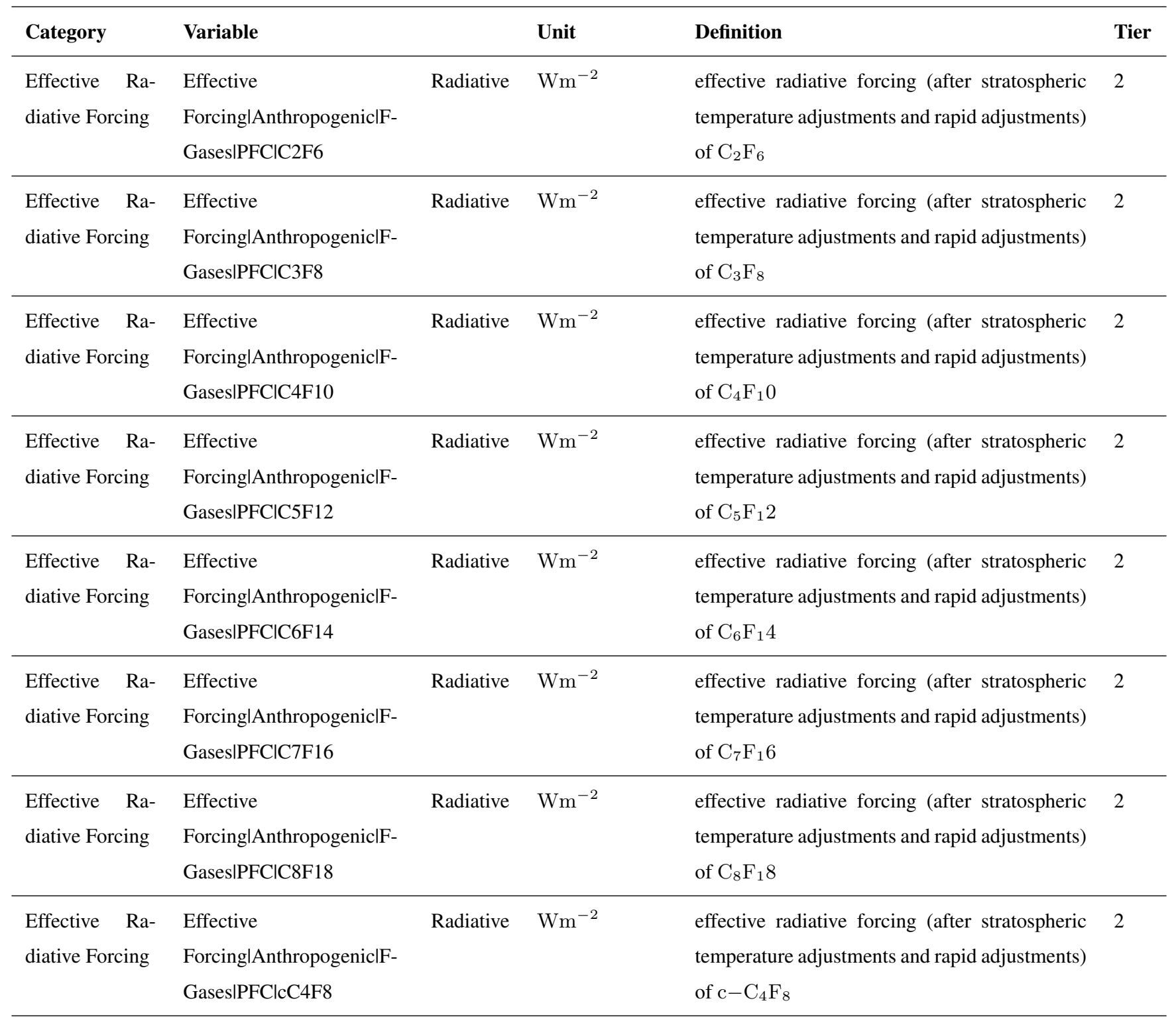


Table S4. Continued.

\begin{tabular}{|c|c|c|c|c|c|}
\hline Category & Variable & & Unit & Definition & Tier \\
\hline $\begin{array}{l}\text { Effective Ra- } \\
\text { diative Forcing }\end{array}$ & $\begin{array}{l}\text { Effective } \\
\text { Forcing|Anthropogenic|F- } \\
\text { Gases|PFC|CF4 }\end{array}$ & Radiative & $\mathrm{Wm}^{-2}$ & $\begin{array}{l}\text { effective radiative forcing (after stratospheric } \\
\text { temperature adjustments and rapid adjustments) } \\
\text { of } \mathrm{CF}_{4}\end{array}$ & 2 \\
\hline $\begin{array}{l}\text { Effective Ra- } \\
\text { diative Forcing }\end{array}$ & $\begin{array}{l}\text { Effective } \\
\text { Forcing|Anthropogenic|F-Gas }\end{array}$ & $\begin{array}{l}\text { Radiative } \\
\text { esISF6 }\end{array}$ & $\mathrm{Wm}^{-2}$ & $\begin{array}{l}\text { effective radiative forcing (after stratospheric } \\
\text { temperature adjustments and rapid adjustments) } \\
\text { of sulfur hexafluoride }\left(\mathrm{SF}_{6}\right)\end{array}$ & 2 \\
\hline $\begin{array}{l}\text { Effective Ra- } \\
\text { diative Forcing }\end{array}$ & $\begin{array}{l}\text { Effective } \\
\text { Forcing|Anthropogenic|F-Gas }\end{array}$ & $\begin{array}{l}\text { Radiative } \\
\text { esISO2F2 }\end{array}$ & $\mathrm{Wm}^{-2}$ & $\begin{array}{l}\text { effective radiative forcing (after stratospheric } \\
\text { temperature adjustments and rapid adjustments) } \\
\text { of sulfuryl fluoride }\left(\mathrm{SO}_{2} \mathrm{~F}_{2}\right)\end{array}$ & 2 \\
\hline $\begin{array}{l}\text { Effective Ra- } \\
\text { diative Forcing }\end{array}$ & $\begin{array}{l}\text { Effective Radiative } \\
\text { inglAnthropogeniclMontreal } \\
\text { Gases|CCl4 }\end{array}$ & Forc- & $\mathrm{Wm}^{-2}$ & $\begin{array}{l}\text { effective radiative forcing (after stratospheric } \\
\text { temperature adjustments and rapid adjustments) } \\
\text { of } \mathrm{CCl}_{4}\end{array}$ & 2 \\
\hline $\begin{array}{l}\text { Effective Ra- } \\
\text { diative Forcing }\end{array}$ & $\begin{array}{l}\text { Effective Radiative } \\
\text { inglAnthropogenic|Montreal } \\
\text { Gases|CFC }\end{array}$ & Forc- & $\mathrm{Wm}^{-2}$ & $\begin{array}{l}\text { effective radiative forcing (after stratospheric } \\
\text { temperature adjustments and rapid adjustments) } \\
\text { of CFC gases (as defined by Table 8.A.1 of } \\
\text { AR5) }\end{array}$ & 2 \\
\hline $\begin{array}{l}\text { Effective Ra- } \\
\text { diative Forcing }\end{array}$ & $\begin{array}{l}\text { Effective Radiative } \\
\text { ing|Anthropogenic|Montreal } \\
\text { Gases|CFC|CFC113 }\end{array}$ & Forc- & $\mathrm{Wm}^{-2}$ & $\begin{array}{l}\text { effective radiative forcing (after stratospheric } \\
\text { temperature adjustments and rapid adjustments) } \\
\text { of } \mathrm{CFC} 113\end{array}$ & 2 \\
\hline
\end{tabular}


Table S4. Continued.

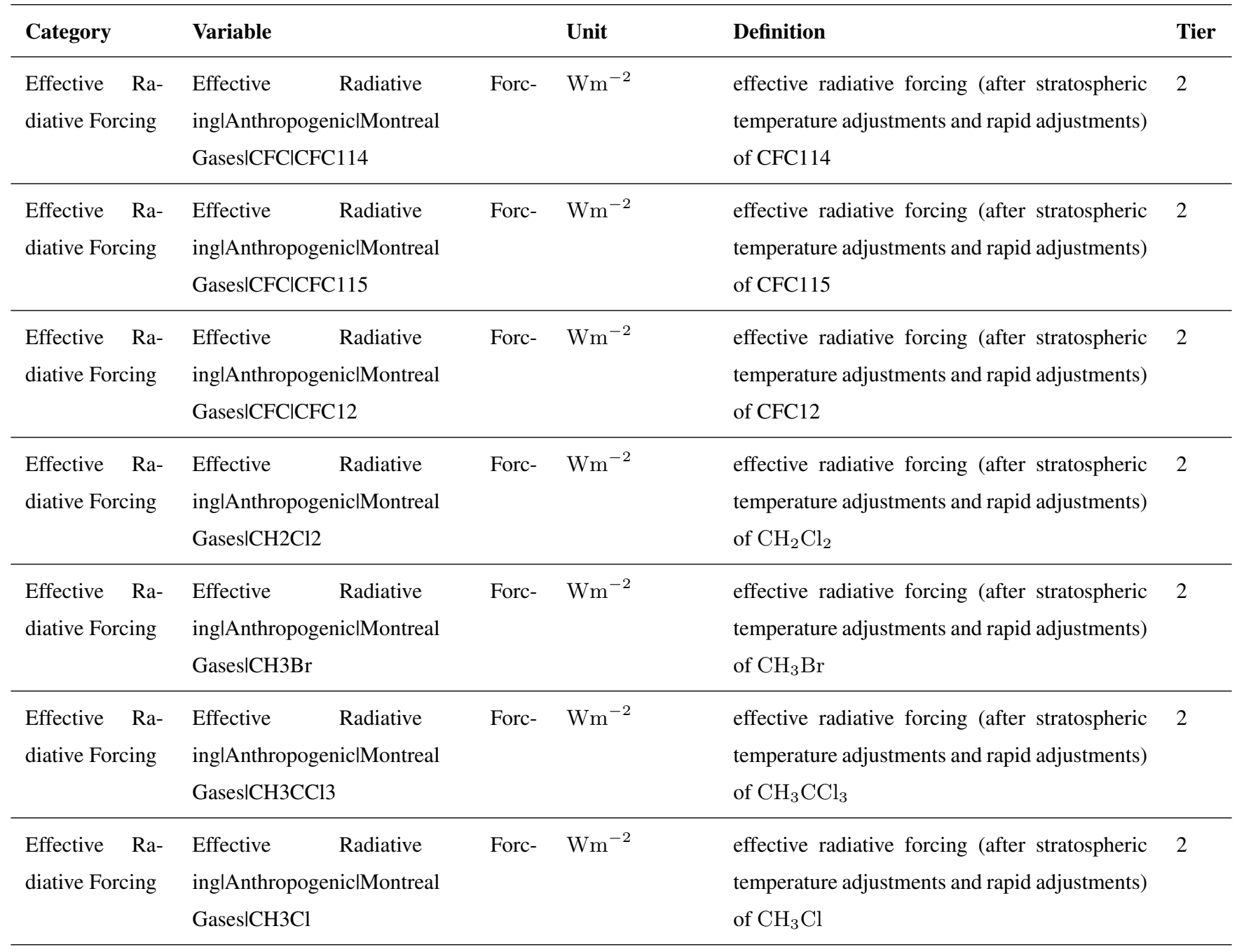


Table S4. Continued.

\begin{tabular}{|c|c|c|c|c|c|}
\hline Category & Variable & & Unit & Definition & Tier \\
\hline $\begin{array}{l}\text { Effective Ra- } \\
\text { diative Forcing }\end{array}$ & $\begin{array}{l}\text { Effective Radiative } \\
\text { inglAnthropogenic|Montreal } \\
\text { Gases|CHCl3 }\end{array}$ & Forc- & $\mathrm{Wm}^{-2}$ & $\begin{array}{l}\text { effective radiative forcing (after stratospheric } \\
\text { temperature adjustments and rapid adjustments) } \\
\text { of } \mathrm{CHCl}_{3}\end{array}$ & 2 \\
\hline $\begin{array}{l}\text { Effective Ra- } \\
\text { diative Forcing }\end{array}$ & $\begin{array}{l}\text { Effective Radiative } \\
\text { inglAnthropogeniclMontreal } \\
\text { Gases|Halon1202 }\end{array}$ & Forc- & $\mathrm{Wm}^{-2}$ & $\begin{array}{l}\text { effective radiative forcing (after stratospheric } \\
\text { temperature adjustments and rapid adjustments) } \\
\text { of Halon-1202 }\end{array}$ & 2 \\
\hline $\begin{array}{l}\text { Effective Ra- } \\
\text { diative Forcing }\end{array}$ & $\begin{array}{l}\text { Effective Radiative } \\
\text { ing|Anthropogenic|Montreal } \\
\text { Gases|Halon1211 }\end{array}$ & Forc- & $\mathrm{Wm}^{-2}$ & $\begin{array}{l}\text { effective radiative forcing (after stratospheric } \\
\text { temperature adjustments and rapid adjustments) } \\
\text { of Halon-1211 }\end{array}$ & 2 \\
\hline $\begin{array}{l}\text { Effective Ra- } \\
\text { diative Forcing }\end{array}$ & $\begin{array}{l}\text { Effective Radiative } \\
\text { inglAnthropogenic|Montreal } \\
\text { Gases|Halon2402 }\end{array}$ & Forc- & $\mathrm{Wm}^{-2}$ & $\begin{array}{l}\text { effective radiative forcing (after stratospheric } \\
\text { temperature adjustments and rapid adjustments) } \\
\text { of Halon- } 2402\end{array}$ & 2 \\
\hline $\begin{array}{l}\text { Effective Ra- } \\
\text { diative Forcing }\end{array}$ & $\begin{array}{l}\text { Effective Radiative } \\
\text { inglAnthropogeniclMontreal } \\
\text { Gases|HCFC141b }\end{array}$ & Forc- & $\mathrm{Wm}^{-2}$ & $\begin{array}{l}\text { effective radiative forcing (after stratospheric } \\
\text { temperature adjustments and rapid adjustments) } \\
\text { of } \mathrm{HCFC} 141 \mathrm{~b}\end{array}$ & 2 \\
\hline
\end{tabular}


Table S4. Continued.

\begin{tabular}{|c|c|c|c|c|c|}
\hline Category & Variable & & Unit & Definition & Tier \\
\hline $\begin{array}{l}\text { Effective Ra- } \\
\text { diative Forcing }\end{array}$ & $\begin{array}{l}\text { Effective Radiative } \\
\text { ing|Anthropogenic|Montreal } \\
\text { Gases|HCFC22 }\end{array}$ & Forc- & $\mathrm{Wm}^{-2}$ & $\begin{array}{l}\text { effective radiative forcing (after stratospheric } \\
\text { temperature adjustments and rapid adjustments) } \\
\text { of } \mathrm{HCFC} 22\end{array}$ & 2 \\
\hline $\begin{array}{l}\text { Effective Ra- } \\
\text { diative Forcing }\end{array}$ & $\begin{array}{l}\text { Effective Radiative } \\
\text { inglAnthropogenic|N2O }\end{array}$ & Forc- & $\mathrm{Wm}^{-2}$ & $\begin{array}{l}\text { effective radiative forcing (after stratospheric } \\
\text { temperature adjustments and rapid adjustments) } \\
\text { of } \mathrm{N}_{2} \mathrm{O}\end{array}$ & 2 \\
\hline $\begin{array}{l}\text { Effective Ra- } \\
\text { diative Forcing }\end{array}$ & $\begin{array}{l}\text { Effective Radiative } \\
\text { inglAnthropogenic|Other }\end{array}$ & Forc- & $\mathrm{Wm}^{-2}$ & $\begin{array}{l}\text { effective radiative forcing from factors not cov- } \\
\text { ered in other categories (after stratospheric tem- } \\
\text { perature adjustments and rapid adjustments) }\end{array}$ & 2 \\
\hline $\begin{array}{l}\text { Effective Ra- } \\
\text { diative Forcing }\end{array}$ & $\begin{array}{l}\text { Effective Radiative } \\
\text { ing|Anthropogenic|Other|Contrails } \\
\text { and Contrail-induced Cirrus }\end{array}$ & Forc- & $\mathrm{Wm}^{-2}$ & $\begin{array}{l}\text { effective radiative forcing from contrails and } \\
\text { contrail-induced cirrus (after stratospheric tem- } \\
\text { perature adjustments and rapid adjustments) }\end{array}$ & 2 \\
\hline $\begin{array}{l}\text { Effective Ra- } \\
\text { diative Forcing }\end{array}$ & $\begin{array}{l}\text { Effective Radiative } \\
\text { inglAnthropogenic|Other|CH4 } \\
\text { dation Stratospheric } \mathrm{H} 2 \mathrm{O}\end{array}$ & $\begin{array}{l}\text { Forc- } \\
\text { Oxi- }\end{array}$ & $\mathrm{Wm}^{-2}$ & $\begin{array}{l}\text { effective radiative forcing from methane oxi- } \\
\text { dation of stratospheric } \mathrm{H} 2 \mathrm{O} \text { (after stratospheric } \\
\text { temperature adjustments and rapid adjustments) }\end{array}$ & 2 \\
\hline
\end{tabular}


Table S4. Continued.

\begin{tabular}{|c|c|c|c|c|}
\hline Category & Variable & Unit & Definition & Tier \\
\hline $\begin{array}{l}\text { Effective Ra- } \\
\text { diative Forcing }\end{array}$ & $\begin{array}{l}\text { Effective Radiative } \quad \text { Forc- } \\
\text { inglAnthropogeniclTropospheric } \\
\text { Ozone }\end{array}$ & $\mathrm{Wm}^{-2}$ & $\begin{array}{l}\text { effective radiative forcing from tropospheric } \\
\text { ozone (after stratospheric temperature adjust- } \\
\text { ments and rapid adjustments) }\end{array}$ & 2 \\
\hline $\begin{array}{l}\text { Effective Ra- } \\
\text { diative Forcing }\end{array}$ & Effective Radiative ForcingINatural & $\mathrm{Wm}^{-2}$ & $\begin{array}{l}\text { effective radiative forcing from all natural } \\
\text { drivers, i.e. solar and volcanic forcing (after } \\
\text { stratospheric temperature adjustments and rapid } \\
\text { adjustments) }\end{array}$ & 2 \\
\hline $\begin{array}{l}\text { Effective Ra- } \\
\text { diative Forcing }\end{array}$ & $\begin{array}{l}\text { Effective Radiative } \\
\text { ing|NaturallSolar }\end{array}$ & $\mathrm{Wm}^{-2}$ & $\begin{array}{l}\text { effective radiative forcing from variations in so- } \\
\text { lar irradience (after stratospheric temperature } \\
\text { adjustments and rapid adjustments) }\end{array}$ & 2 \\
\hline Emissions & Emissions $\mid \mathrm{BC}$ & $\mathrm{MtBCyr}^{-1}$ & total black carbon emissions & 1 \\
\hline Emissions & Emissions/BCIMAGICC AFOLU & $\mathrm{MtBCyr}^{-1}$ & $\begin{array}{l}\text { black carbon emissions from agriculture, } \\
\text { forestry and other land use (IPCC category 3), } \\
\text { excluding any fossil-fuel based emissions in } \\
\text { the Agricultural sector (hence not identical to } \\
\text { WG3 AFOLU) }\end{array}$ & 2 \\
\hline Emissions & Emissions|BClOther & $\mathrm{MtBCyr}^{-1}$ & $\begin{array}{l}\text { black carbon emissions from other sources } \\
\text { (please provide a definition of other sources in } \\
\text { this category in the 'comments' tab) }\end{array}$ & 2 \\
\hline Emissions & Emissions|CH4 & $\mathrm{MtCH}_{4} \mathrm{yr}^{-1}$ & total methane emissions & 1 \\
\hline Emissions & Emissions|CH4IMAGICC AFOLU & $\mathrm{MtCH}_{4} \mathrm{yr}^{-1}$ & $\begin{array}{l}\text { methane emissions from agriculture, forestry } \\
\text { and other land use (IPCC category 3), exclud- } \\
\text { ing any fossil-fuel based emissions in the Agri- } \\
\text { cultural sector (hence not identical to WG3 } \\
\text { AFOLU) }\end{array}$ & 2 \\
\hline
\end{tabular}


Table S4. Continued.

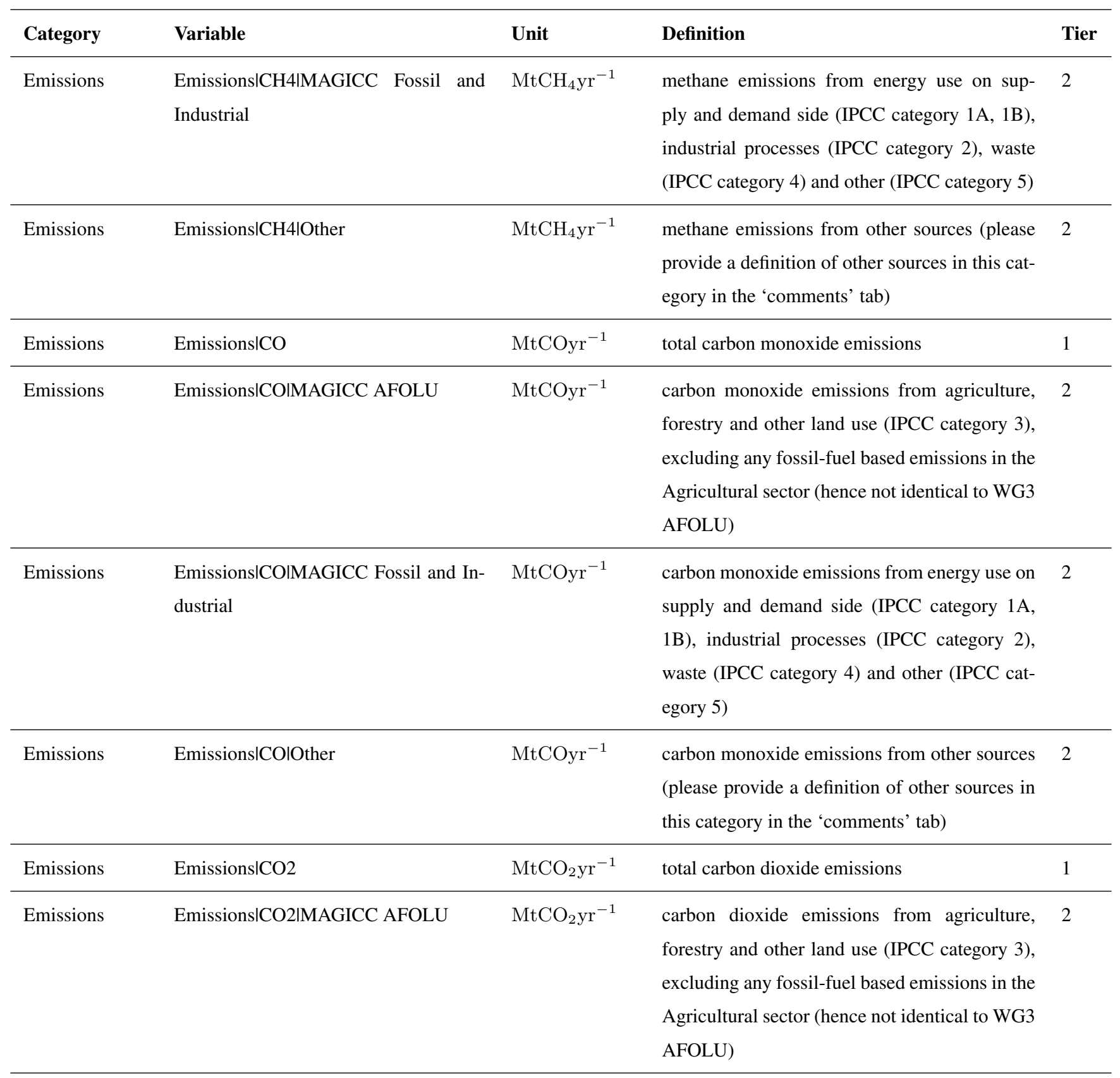


Table S4. Continued.

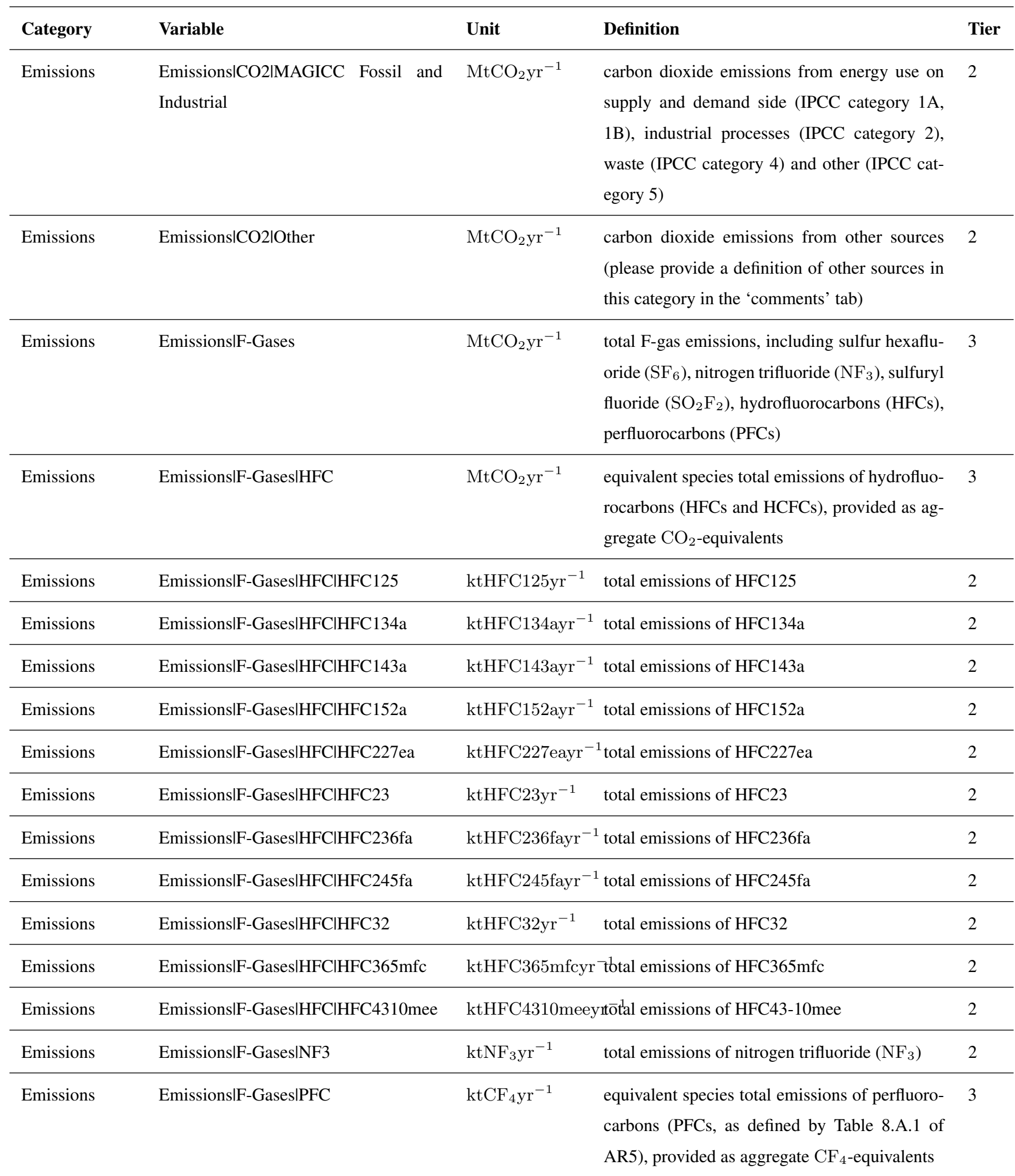


Table S4. Continued.

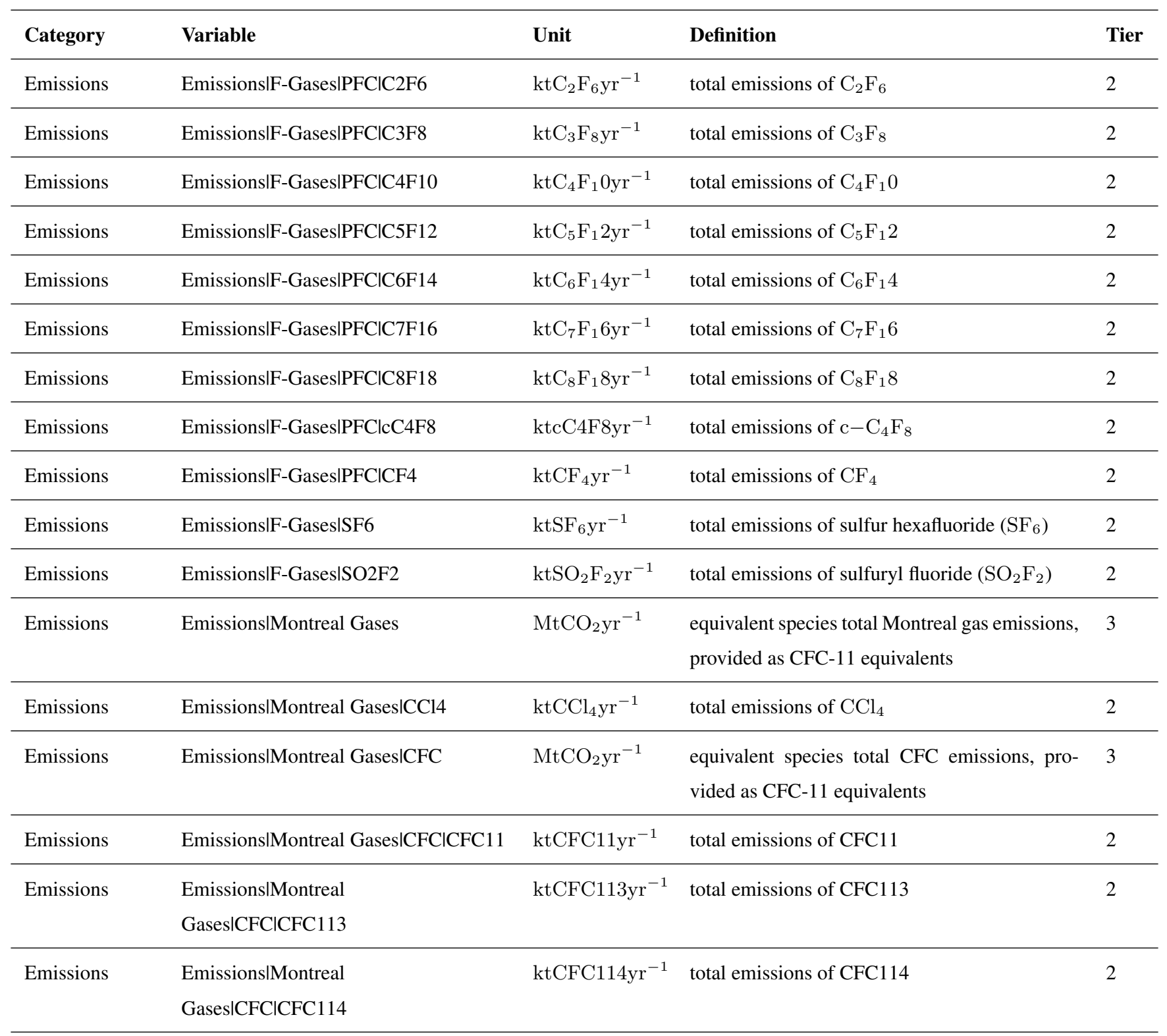


Table S4. Continued.

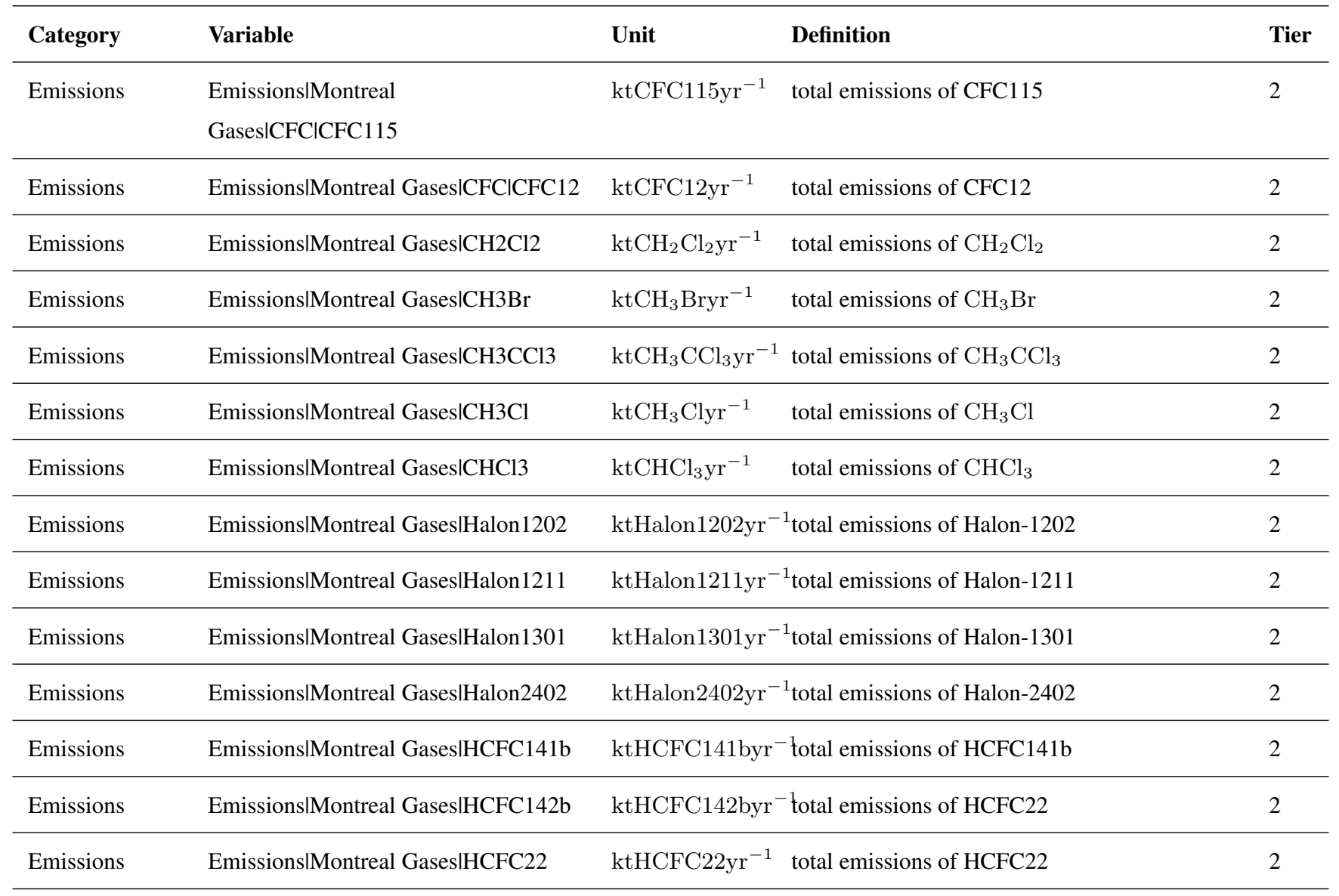


Table S4. Continued.

\begin{tabular}{llll}
\hline Category & Variable & Unit & Definition \\
\hline Emissions & EmissionsIN2O & $\mathrm{ktN}_{2} \mathrm{Oyr}^{-1}$ & Total nitrogen emissions \\
\hline Emissions & EmissionsIN2OIMAGICC AFOLU & $\mathrm{ktN}_{2} \mathrm{Oyr}^{-1}$ & $\begin{array}{l}\text { nitrogen emissions from agriculture, forestry } \\
\text { and other land use (IPCC category 3), exclud- } \\
\text { ing any fossil-fuel based emissions in the Agri- } \\
\text { cultural sector (hence not identical to WG3 } \\
\text { AFOLU) }\end{array}$ \\
\hline
\end{tabular}

\begin{tabular}{llll}
\hline Emissions & $\begin{array}{l}\text { EmissionsIN2OIMAGICC Fossil and } \mathrm{ktN}_{2} \mathrm{Oyr}^{-1} \\
\text { Industrial }\end{array}$ & & $\begin{array}{l}\text { nitrogen emissions from energy use on sup- } \\
\text { ply and demand side (IPCC category 1A, 1B), }\end{array}$ \\
& & industrial processes (IPCC category 2), waste \\
& & (IPCC category 4) and other (IPCC category 5) \\
\hline
\end{tabular}

\begin{tabular}{|c|c|c|c|c|}
\hline Emissions & Emissions 1 N2OlOther & $\mathrm{ktN}_{2} \mathrm{Oyr}^{-1}$ & $\begin{array}{l}\text { nitrogen emissions from other sources (please } \\
\text { provide a definition of other sources in this cat- } \\
\text { egory in the 'comments' tab) }\end{array}$ & 2 \\
\hline Emissions & Emissions $\mid \mathrm{NH} 3$ & $\mathrm{MtNH} \mathrm{yr}^{-1}$ & total ammonia emissions & 1 \\
\hline Emissions & Emissions|NH3|MAGICC AFOLU & MtNH3yr $^{-1}$ & $\begin{array}{l}\text { ammonia emissions from agriculture, forestry } \\
\text { and other land use (IPCC category 3), exclud- } \\
\text { ing any fossil-fuel based emissions in the Agri- } \\
\text { cultural sector (hence not identical to WG3 } \\
\text { AFOLU) }\end{array}$ & 2 \\
\hline Emissions & Emissions|NH3|Other & $\mathrm{MtNH}_{3} \mathrm{yr}^{-1}$ & $\begin{array}{l}\text { ammonia emissions from other sources (please } \\
\text { provide a definition of other sources in this cat- } \\
\text { egory in the 'comments' tab) }\end{array}$ & 2 \\
\hline
\end{tabular}


Table S4. Continued.

\begin{tabular}{|c|c|c|c|c|}
\hline Category & Variable & Unit & Definition & Tier \\
\hline Emissions & EmissionslNOx & $\mathrm{MtNOxyr}^{-1}$ & total nitrous oxide emissions & 1 \\
\hline Emissions & $\begin{array}{l}\text { EmissionsINOxIMAGICC Fossil and } \\
\text { Industrial }\end{array}$ & $\mathrm{MtNOxyr}^{-1}$ & $\begin{array}{l}\text { nitrous oxide emissions from energy use on } \\
\text { supply and demand side (IPCC category 1A, } \\
\text { 1B), industrial processes (IPCC category 2), } \\
\text { waste (IPCC category 4) and other (IPCC cat- } \\
\text { egory 5) }\end{array}$ & 2 \\
\hline Emissions & Emissions|NOx|Other & $\mathrm{MtNOxyr}^{-1}$ & $\begin{array}{l}\text { nitrous oxide emissions from other sources } \\
\text { (please provide a definition of other sources in } \\
\text { this category in the 'comments' tab) }\end{array}$ & 2 \\
\hline Emissions & Emissions $\mid \mathrm{OC}$ & $\mathrm{MtOCyr}^{-1}$ & total organic carbon emissions & 1 \\
\hline Emissions & Emissions|OCIMAGICC AFOLU & $\mathrm{MtOCyr}^{-1}$ & $\begin{array}{l}\text { organic carbon emissions from agriculture, } \\
\text { forestry and other land use (IPCC category 3), } \\
\text { excluding any fossil-fuel based emissions in the } \\
\text { Agricultural sector (hence not identical to WG3 } \\
\text { AFOLU) }\end{array}$ & 2 \\
\hline Emissions & Emissions $|\mathrm{OC}| \mathrm{Other}$ & $\mathrm{MtOCyr}^{-1}$ & $\begin{array}{l}\text { organic carbon emissions from other sources } \\
\text { (please provide a definition of other sources in } \\
\text { this category in the 'comments' tab) }\end{array}$ & 2 \\
\hline
\end{tabular}


Table S4. Continued.

\begin{tabular}{|c|c|c|c|c|}
\hline Category & Variable & Unit & Definition & Tier \\
\hline Emissions & EmissionsISulfur & $\mathrm{MtSO}_{2} \mathrm{yr}^{-1}$ & $\begin{array}{l}\text { total sulfur (as a precursor for sulfates) emis- } \\
\text { sions }\end{array}$ & 1 \\
\hline Emissions & EmissionsISulfurlMAGICC AFOLU & $\mathrm{MtSO}_{2} \mathrm{yr}^{-1}$ & $\begin{array}{l}\text { sulfur (as a precursor for sulfates) emissions } \\
\text { from agriculture, forestry and other land use } \\
\text { (IPCC category 3), excluding any fossil-fuel } \\
\text { based emissions in the Agricultural sector } \\
\text { (hence not identical to WG3 AFOLU) }\end{array}$ & 2 \\
\hline Emissions & $\begin{array}{l}\text { Emissions|Sulfur|MAGICC Fossil and } \\
\text { Industrial }\end{array}$ & $\mathrm{MtSO}_{2} \mathrm{yr}^{-1}$ & $\begin{array}{l}\text { sulfur (as a precursor for sulfates) emissions } \\
\text { from energy use on supply and demand side } \\
\text { (IPCC category 1A, 1B), industrial processes } \\
\text { (IPCC category 2), waste (IPCC category 4) and } \\
\text { other (IPCC category 5) }\end{array}$ & 2 \\
\hline Emissions & Emissions|Sulfur|Other & $\mathrm{MtSO}_{2} \mathrm{yr}^{-1}$ & $\begin{array}{l}\text { sulfur (as a precursor for sulfates) emissions } \\
\text { from other sources (please provide a definition } \\
\text { of other sources in this category in the 'com- } \\
\text { ments' tab) }\end{array}$ & 2 \\
\hline Emissions & Emissions/VOC & $\mathrm{MtVOCyr}^{-1}$ & $\begin{array}{l}\text { total (non-methane) volatile organic com- } \\
\text { pounds emissions }\end{array}$ & 1 \\
\hline Emissions & $\begin{array}{l}\text { Emissions|VOCIMAGICC Fossil and } \\
\text { Industrial }\end{array}$ & $\mathrm{MtVOCyr}^{-1}$ & $\begin{array}{l}\text { (non-methane) volatile organic compounds } \\
\text { emissions from energy use on supply and de- } \\
\text { mand side (IPCC category 1A, 1B), industrial } \\
\text { processes (IPCC category 2), waste (IPCC cat- } \\
\text { egory } 4 \text { ) and other (IPCC category 5) }\end{array}$ & 2 \\
\hline
\end{tabular}


Table S4. Continued.

\begin{tabular}{|c|c|c|c|c|}
\hline Category & Variable & Unit & Definition & Tier \\
\hline Emissions & EmissionsIVOCIOther & $\mathrm{MtVOCyr}^{-1}$ & $\begin{array}{l}\text { (non-methane) volatile organic compounds } \\
\text { emissions from other sources (please provide a } \\
\text { definition of other sources in this category in the } \\
\text { 'comments' tab) }\end{array}$ & 2 \\
\hline Nitrogen Cycle & Atmospheric LifetimelN2O & $\mathrm{yr}$ & total atmospheric lifetime of nitrogen & 3 \\
\hline Ocean & Ocean $\mathrm{pH}$ & Dimensionless & $\mathrm{pH}$ of the ocean's surface layer & 3 \\
\hline $\begin{array}{l}\text { Radiative Forc- } \\
\text { ing }\end{array}$ & Radiative Forcing & $\mathrm{Wm}^{-2}$ & $\begin{array}{l}\text { radiative forcing from all anthropogenic and } \\
\text { natural sources (after stratospheric temperature } \\
\text { adjustments) }\end{array}$ & 1 \\
\hline $\begin{array}{l}\text { Radiative Forc- } \\
\text { ing }\end{array}$ & Radiative Forcing|Anthropogenic & $\mathrm{Wm}^{-2}$ & $\begin{array}{l}\text { radiative forcing from all anthropogenic sources } \\
\text { (after stratospheric temperature adjustments) }\end{array}$ & 1 \\
\hline $\begin{array}{l}\text { Radiative Forc- } \\
\text { ing }\end{array}$ & $\begin{array}{ll}\text { Radiative } & \text { Forc- } \\
\text { inglAnthropogeniclAerosols } & \end{array}$ & $\mathrm{Wm}^{-2}$ & $\begin{array}{l}\text { radiative forcing from aerosols (after strato- } \\
\text { spheric temperature adjustments) }\end{array}$ & 1 \\
\hline $\begin{array}{l}\text { Radiative Forc- } \\
\text { ing }\end{array}$ & $\begin{array}{l}\text { Radiative } \\
\text { Forcing|Anthropogenic|Aerosols|Aerosols- } \\
\text { cloud Interactions }\end{array}$ & $\mathrm{Wm}^{-2}$ & $\begin{array}{l}\text { radiative forcing from indirect effects of } \\
\text { aerosols on clouds (after stratospheric temper- } \\
\text { ature adjustments) }\end{array}$ & 2 \\
\hline $\begin{array}{l}\text { Radiative Forc- } \\
\text { ing }\end{array}$ & $\begin{array}{l}\text { Radiative } \\
\text { Forcing|Anthropogenic|Aerosols|Aerosols- } \\
\text { radiation Interactions|BC and OC|BC }\end{array}$ & $\mathrm{Wm}^{-2}$ & $\begin{array}{l}\text { radiative forcing from aerosol-radiative effects } \\
\text { from black carbon emissions (after strato- } \\
\text { spheric temperature adjustments) }\end{array}$ & 2 \\
\hline $\begin{array}{l}\text { Radiative Forc- } \\
\text { ing }\end{array}$ & $\begin{array}{l}\text { Radiative } \\
\text { Forcing|Anthropogenic|Aerosols|Aerosols- } \\
\text { radiation Interactions|BC and } \\
\text { OC|BC|Biomass Burning }\end{array}$ & $\mathrm{Wm}^{-2}$ & $\begin{array}{l}\text { radiative forcing from aerosol-radiative effects } \\
\text { from black carbon biomass burning emissions } \\
\text { (after stratospheric temperature adjustments) }\end{array}$ & 2 \\
\hline
\end{tabular}


Table S4. Continued.

\begin{tabular}{|c|c|c|c|c|}
\hline Category & Variable & Unit & Definition & Tier \\
\hline $\begin{array}{l}\text { Radiative Forc- } \\
\text { ing }\end{array}$ & $\begin{array}{l}\text { Radiative } \\
\text { Forcing|Anthropogenic|Aerosols|Aerosols- } \\
\text { radiation Interactions|BC and } \\
\text { OC|BC|Fossil and Industrial }\end{array}$ & $\mathrm{Wm}^{-2}$ & $\begin{array}{l}\text { radiative forcing from aerosol-radiative effects } \\
\text { from black carbon fossil and industrial emis- } \\
\text { sions (after stratospheric temperature adjust- } \\
\text { ments) }\end{array}$ & 2 \\
\hline $\begin{array}{l}\text { Radiative Forc- } \\
\text { ing }\end{array}$ & $\begin{array}{l}\text { Radiative } \\
\text { Forcing|Anthropogenic|Aerosols|Aerosols- } \\
\text { radiation Interactions|BC and } \\
\text { OCIOC|Biomass Burning }\end{array}$ & $\mathrm{Wm}^{-2}$ & $\begin{array}{l}\text { radiative forcing from aerosol-radiative effects } \\
\text { from organic carbon biomass burning emissions } \\
\text { (after stratospheric temperature adjustments) }\end{array}$ & 2 \\
\hline $\begin{array}{l}\text { Radiative Forc- } \\
\text { ing }\end{array}$ & $\begin{array}{l}\text { Radiative } \\
\text { Forcing|Anthropogenic|Aerosols|Aerosols- } \\
\text { radiation Interactions|BC and } \\
\text { OCIOC|Fossil and Industrial }\end{array}$ & $\mathrm{Wm}^{-2}$ & $\begin{array}{l}\text { radiative forcing from aerosol-radiative effects } \\
\text { from organic carbon fossil and industrial emis- } \\
\text { sions (after stratospheric temperature adjust- } \\
\text { ments) }\end{array}$ & 2 \\
\hline $\begin{array}{l}\text { Radiative Forc- } \\
\text { ing }\end{array}$ & $\begin{array}{l}\text { Radiative } \\
\text { Forcing|Anthropogenic|Aerosols|Aerosols- } \\
\text { radiation Interactions|Biomass Burning }\end{array}$ & $\mathrm{Wm}^{-2}$ & $\begin{array}{l}\text { radiative forcing from aerosol-radiative effects } \\
\text { from biomass burning emissions (after strato- } \\
\text { spheric temperature adjustments) }\end{array}$ & 2 \\
\hline
\end{tabular}


Table S4. Continued.

\begin{tabular}{|c|c|c|c|c|}
\hline Category & Variable & Unit & Definition & Tier \\
\hline $\begin{array}{l}\text { Radiative Forc- } \\
\text { ing }\end{array}$ & $\begin{array}{l}\text { Radiative } \\
\text { Forcing|Anthropogenic|Aerosols|Aerosols- } \\
\text { radiation Interactions|Biomass Burn- } \\
\text { ing|BC and OC }\end{array}$ & $\mathrm{Wm}^{-2}$ & $\begin{array}{l}\text { radiative forcing from aerosol-radiative effects } \\
\text { from black and organic carbon biomass burn- } \\
\text { ing emissions (after stratospheric temperature } \\
\text { adjustments) }\end{array}$ & 2 \\
\hline $\begin{array}{l}\text { Radiative Forc- } \\
\text { ing }\end{array}$ & $\begin{array}{l}\text { Radiative } \\
\text { Forcing|Anthropogenic|Aerosols|Aerosols- } \\
\text { radiation Interactions|Biomass Burn- } \\
\text { ing|BC and OCIOC }\end{array}$ & $\mathrm{Wm}^{-2}$ & $\begin{array}{l}\text { radiative forcing from aerosol-radiative effects } \\
\text { from organic carbon biomass burning emissions } \\
\text { (after stratospheric temperature adjustments) }\end{array}$ & 2 \\
\hline $\begin{array}{l}\text { Radiative Forc- } \\
\text { ing }\end{array}$ & $\begin{array}{l}\text { Radiative } \\
\text { Forcing|Anthropogenic|Aerosols|Aerosols- } \\
\text { radiation Interactions|Biomass Burn- } \\
\text { inglNH3 }\end{array}$ & $\mathrm{Wm}^{-2}$ & $\begin{array}{l}\text { radiative forcing from aerosol-radiative effects } \\
\text { from ammonia biomass burning emissions (af- } \\
\text { ter stratospheric temperature adjustments) }\end{array}$ & 2 \\
\hline $\begin{array}{l}\text { Radiative Forc- } \\
\text { ing }\end{array}$ & $\begin{array}{l}\text { Radiative } \\
\text { Forcing|Anthropogenic|Aerosols|Aerosols- } \\
\text { radiation Interactions|Biomass Burn- } \\
\text { inglNitrate }\end{array}$ & $\mathrm{Wm}^{-2}$ & $\begin{array}{l}\text { radiative forcing from aerosol-radiative effects } \\
\text { from nitrate biomass burning emissions (after } \\
\text { stratospheric temperature adjustments) }\end{array}$ & 2 \\
\hline
\end{tabular}


Table S4. Continued.

\begin{tabular}{|c|c|c|c|c|}
\hline Category & Variable & Unit & Definition & Tier \\
\hline $\begin{array}{l}\text { Radiative Forc- } \\
\text { ing }\end{array}$ & $\begin{array}{l}\text { Radiative } \\
\text { Forcing|Anthropogenic|Aerosols|Aerosols- } \\
\text { radiation Interactions|Biomass Burn- } \\
\text { ing|Sulfate }\end{array}$ & $\mathrm{Wm}^{-2}$ & $\begin{array}{l}\text { radiative forcing from aerosol-radiative effects } \\
\text { from sulfate biomass burning emissions (after } \\
\text { stratospheric temperature adjustments) }\end{array}$ & 2 \\
\hline $\begin{array}{l}\text { Radiative Forc- } \\
\text { ing }\end{array}$ & $\begin{array}{l}\text { Radiative } \\
\text { Forcing|Anthropogenic|Aerosols|Aerosols- } \\
\text { radiation Interactions|Fossil and } \\
\text { Industrial|BC and OCIBC }\end{array}$ & $\mathrm{Wm}^{-2}$ & $\begin{array}{l}\text { radiative forcing from aerosol-radiative effects } \\
\text { from black carbon fossil and industrial emis- } \\
\text { sions (after stratospheric temperature adjust- } \\
\text { ments) }\end{array}$ & 2 \\
\hline $\begin{array}{l}\text { Radiative Forc- } \\
\text { ing }\end{array}$ & $\begin{array}{l}\text { Radiative } \\
\text { Forcing|Anthropogenic|Aerosols|Aerosols- } \\
\text { radiation Interactions|Fossil and } \\
\text { Industrial|BC and OCIOC }\end{array}$ & $\mathrm{Wm}^{-2}$ & $\begin{array}{l}\text { radiative forcing from aerosol-radiative effects } \\
\text { from organic carbon fossil and industrial emis- } \\
\text { sions (after stratospheric temperature adjust- } \\
\text { ments) }\end{array}$ & 2 \\
\hline
\end{tabular}


Table S4. Continued.

\begin{tabular}{|c|c|c|c|c|}
\hline Category & Variable & Unit & Definition & Tier \\
\hline $\begin{array}{l}\text { Radiative Forc- } \\
\text { ing }\end{array}$ & $\begin{array}{l}\text { Radiative } \\
\text { Forcing|Anthropogenic|Aerosols|Aerosols- } \\
\text { radiation Interactions|Fossil and } \\
\text { Industrial|NH3 }\end{array}$ & $\mathrm{Wm}^{-2}$ & $\begin{array}{l}\text { radiative forcing from aerosol-radiative effects } \\
\text { from ammonia fossil and industrial emissions } \\
\text { (after stratospheric temperature adjustments) }\end{array}$ & 2 \\
\hline $\begin{array}{l}\text { Radiative Forc- } \\
\text { ing }\end{array}$ & $\begin{array}{l}\text { Radiative } \\
\text { Forcing|Anthropogenic|Aerosols|Aerosols- } \\
\text { radiation Interactions|Mineral Dust }\end{array}$ & $\mathrm{Wm}^{-2}$ & $\begin{array}{l}\text { radiative forcing from aerosol-radiative effects } \\
\text { from mineral dust emissions (after stratospheric } \\
\text { temperature adjustments) }\end{array}$ & 2 \\
\hline $\begin{array}{l}\text { Radiative Forc- } \\
\text { ing }\end{array}$ & $\begin{array}{l}\text { Radiative } \\
\text { Forcing|Anthropogenic|Aerosols|Aerosols- } \\
\text { radiation Interactions } \mid \mathrm{NH} 3\end{array}$ & $\mathrm{Wm}^{-2}$ & $\begin{array}{l}\text { radiative forcing from aerosol-radiative effects } \\
\text { from ammonia emissions (after stratospheric } \\
\text { temperature adjustments) }\end{array}$ & 2 \\
\hline
\end{tabular}


Table S4. Continued.

\begin{tabular}{|c|c|c|c|c|}
\hline Category & Variable & Unit & Definition & Tier \\
\hline $\begin{array}{l}\text { Radiative Forc- } \\
\text { ing }\end{array}$ & $\begin{array}{l}\text { Radiative } \\
\text { Forcing|Anthropogenic|Aerosols|Aerosols- } \\
\text { radiation InteractionsINH3|Biomass } \\
\text { Burning }\end{array}$ & $\mathrm{Wm}^{-2}$ & $\begin{array}{l}\text { radiative forcing from aerosol-radiative effects } \\
\text { from ammonia biomass burning emissions (af- } \\
\text { ter stratospheric temperature adjustments) }\end{array}$ & 2 \\
\hline $\begin{array}{l}\text { Radiative Forc- } \\
\text { ing }\end{array}$ & $\begin{array}{l}\text { Radiative } \\
\text { Forcing|Anthropogenic|Aerosols|Aerosols- } \\
\text { radiation Interactions|NitratelBiomass } \\
\text { Burning }\end{array}$ & $\mathrm{Wm}^{-2}$ & $\begin{array}{l}\text { radiative forcing from aerosol-radiative effects } \\
\text { from nitrate biomass burning emissions (after } \\
\text { stratospheric temperature adjustments) }\end{array}$ & 2 \\
\hline $\begin{array}{l}\text { Radiative Forc- } \\
\text { ing }\end{array}$ & $\begin{array}{l}\text { Radiative } \\
\text { Forcing|Anthropogenic|Aerosols|Aerosols- } \\
\text { radiation Interactions|Nitrate|Fossil and } \\
\text { Industrial }\end{array}$ & $\mathrm{Wm}^{-2}$ & $\begin{array}{l}\text { radiative forcing from aerosol-radiative effects } \\
\text { from nitrate fossil and industrial emissions (af- } \\
\text { ter stratospheric temperature adjustments) }\end{array}$ & 2 \\
\hline
\end{tabular}


Table S4. Continued.

\begin{tabular}{|c|c|c|c|c|}
\hline Category & Variable & Unit & Definition & Tier \\
\hline $\begin{array}{l}\text { Radiative Forc- } \\
\text { ing }\end{array}$ & $\begin{array}{l}\text { Radiative } \\
\text { Forcing|Anthropogenic|Aerosols|Aerosols- } \\
\text { radiation Interactions|Other }\end{array}$ & $\mathrm{Wm}^{-2}$ & $\begin{array}{l}\text { radiative forcing from aerosol-radiative effects } \\
\text { not covered in the other categories (after strato- } \\
\text { spheric temperature adjustments) (please spec- } \\
\text { ify in comments) }\end{array}$ & 2 \\
\hline $\begin{array}{l}\text { Radiative Forc- } \\
\text { ing }\end{array}$ & $\begin{array}{l}\text { Radiative } \\
\text { Forcing|Anthropogenic|Aerosols|Aerosols- } \\
\text { radiation Interactions|Sulfate|Biomass } \\
\text { Burning }\end{array}$ & $\mathrm{Wm}^{-2}$ & $\begin{array}{l}\text { radiative forcing from aerosol-radiative effects } \\
\text { from sulfate biomass burning emissions (after } \\
\text { stratospheric temperature adjustments) }\end{array}$ & 2 \\
\hline $\begin{array}{l}\text { Radiative Forc- } \\
\text { ing }\end{array}$ & $\begin{array}{l}\text { Radiative } \\
\text { Forcing|Anthropogenic|Aerosols|Aerosols- } \\
\text { radiation Interactions|Sulfate|Fossil and } \\
\text { Industrial }\end{array}$ & $\mathrm{Wm}^{-2}$ & $\begin{array}{l}\text { radiative forcing from aerosol-radiative effects } \\
\text { from sulfate fossil and industrial emissions (af- } \\
\text { ter stratospheric temperature adjustments) }\end{array}$ & 2 \\
\hline $\begin{array}{l}\text { Radiative Forc- } \\
\text { ing }\end{array}$ & $\begin{array}{l}\text { Radiative Forc- } \\
\text { inglAnthropogeniclAlbedo Change }\end{array}$ & $\mathrm{Wm}^{-2}$ & $\begin{array}{l}\text { radiative forcing from albedo change (after } \\
\text { stratospheric temperature adjustments) }\end{array}$ & 2 \\
\hline $\begin{array}{l}\text { Radiative Forc- } \\
\text { ing }\end{array}$ & Radiative Forcing|Anthropogenic|CH4 & $\mathrm{Wm}^{-2}$ & $\begin{array}{l}\text { radiative forcing (after stratospheric tempera- } \\
\text { ture adjustments) of } \mathrm{CH}_{4}\end{array}$ & 2 \\
\hline
\end{tabular}


Table S4. Continued.

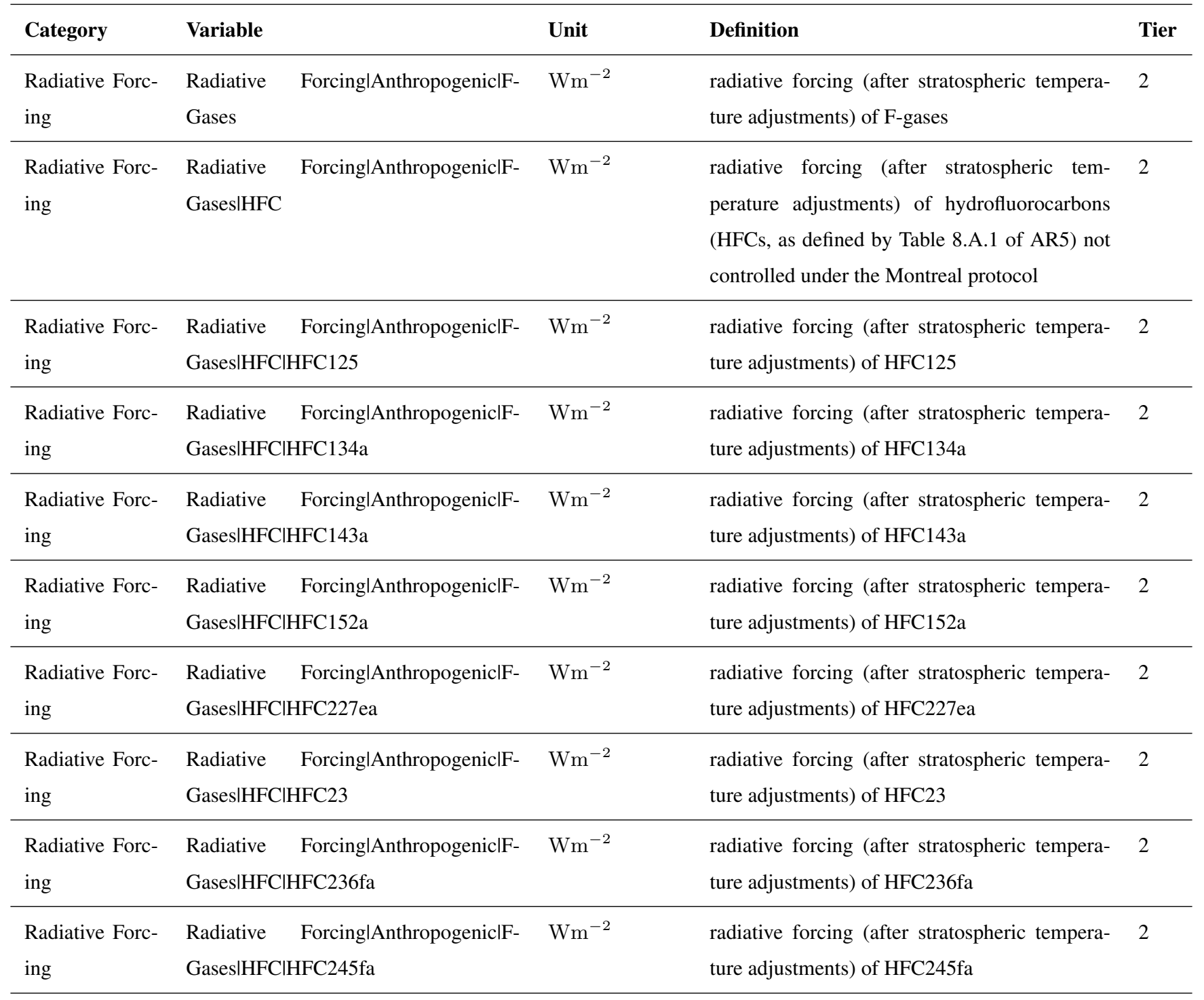


Table S4. Continued.

\begin{tabular}{|c|c|c|c|c|}
\hline Category & Variable & Unit & Definition & Tier \\
\hline $\begin{array}{l}\text { Radiative Forc- } \\
\text { ing }\end{array}$ & $\begin{array}{l}\text { Radiative ForcinglAnthropogenic|F- } \\
\text { Gases|HFCIHFC32 }\end{array}$ & $\mathrm{Wm}^{-2}$ & $\begin{array}{l}\text { radiative forcing (after stratospheric tempera- } \\
\text { ture adjustments) of HFC32 }\end{array}$ & 2 \\
\hline $\begin{array}{l}\text { Radiative Forc- } \\
\text { ing }\end{array}$ & $\begin{array}{l}\text { Radiative ForcinglAnthropogenic|F- } \\
\text { Gases|HFCIHFC365mfc }\end{array}$ & $\mathrm{Wm}^{-2}$ & $\begin{array}{l}\text { radiative forcing (after stratospheric tempera- } \\
\text { ture adjustments) of } \mathrm{HFC} 365 \mathrm{mfc}\end{array}$ & 2 \\
\hline $\begin{array}{l}\text { Radiative Forc- } \\
\text { ing }\end{array}$ & $\begin{array}{l}\text { Radiative ForcinglAnthropogenic|F- } \\
\text { Gases|HFCIHFC4310mee }\end{array}$ & $\mathrm{Wm}^{-2}$ & $\begin{array}{l}\text { radiative forcing (after stratospheric tempera- } \\
\text { ture adjustments) of HFC43-10mee }\end{array}$ & 2 \\
\hline $\begin{array}{l}\text { Radiative Forc- } \\
\text { ing }\end{array}$ & $\begin{array}{ll}\text { Radiative } & \text { ForcinglAnthropogenic|F- } \\
\text { GasesINF3 } & \end{array}$ & $\mathrm{Wm}^{-2}$ & $\begin{array}{l}\text { radiative forcing (after stratospheric tempera- } \\
\text { ture adjustments) of nitrogen trifluoride }\left(\mathrm{NF}_{3}\right)\end{array}$ & 2 \\
\hline $\begin{array}{l}\text { Radiative Forc- } \\
\text { ing }\end{array}$ & $\begin{array}{l}\text { Radiative Forcing|Anthropogenic|F- } \\
\text { GasesIPFCIC2F6 }\end{array}$ & $\mathrm{Wm}^{-2}$ & $\begin{array}{l}\text { radiative forcing (after stratospheric tempera- } \\
\text { ture adjustments) of } \mathrm{C}_{2} \mathrm{~F}_{6}\end{array}$ & 2 \\
\hline $\begin{array}{l}\text { Radiative Forc- } \\
\text { ing }\end{array}$ & $\begin{array}{l}\text { Radiative Forcing|Anthropogenic|F- } \\
\text { Gases|PFCIC3F8 }\end{array}$ & $\mathrm{Wm}^{-2}$ & $\begin{array}{l}\text { radiative forcing (after stratospheric tempera- } \\
\text { ture adjustments) of } \mathrm{C}_{3} \mathrm{~F}_{8}\end{array}$ & 2 \\
\hline $\begin{array}{l}\text { Radiative Forc- } \\
\text { ing }\end{array}$ & $\begin{array}{l}\text { Radiative Forcing|Anthropogenic|F- } \\
\text { Gases|PFCIC4F10 }\end{array}$ & $\mathrm{Wm}^{-2}$ & $\begin{array}{l}\text { radiative forcing (after stratospheric tempera- } \\
\text { ture adjustments) of } \mathrm{C}_{4} \mathrm{~F}_{1} 0\end{array}$ & 2 \\
\hline
\end{tabular}


Table S4. Continued.

\begin{tabular}{|c|c|c|c|c|}
\hline Category & Variable & Unit & Definition & Tier \\
\hline $\begin{array}{l}\text { Radiative Forc- } \\
\text { ing }\end{array}$ & $\begin{array}{l}\text { Radiative ForcinglAnthropogenic|F- } \\
\text { Gases|PFCIC7F16 }\end{array}$ & $\mathrm{Wm}^{-2}$ & $\begin{array}{l}\text { radiative forcing (after stratospheric tempera- } \\
\text { ture adjustments) of } \mathrm{C}_{7} \mathrm{~F}_{1} 6\end{array}$ & 2 \\
\hline $\begin{array}{l}\text { Radiative Forc- } \\
\text { ing }\end{array}$ & $\begin{array}{l}\text { Radiative ForcinglAnthropogenic|F- } \\
\text { Gases|PFClC8F18 }\end{array}$ & $\mathrm{Wm}^{-2}$ & $\begin{array}{l}\text { radiative forcing (after stratospheric tempera- } \\
\text { ture adjustments) of } \mathrm{C}_{8} \mathrm{~F}_{1} 8\end{array}$ & 2 \\
\hline $\begin{array}{l}\text { Radiative Forc- } \\
\text { ing }\end{array}$ & $\begin{array}{l}\text { Radiative ForcinglAnthropogenic|F- } \\
\text { Gases|PFCICF4 }\end{array}$ & $\mathrm{Wm}^{-2}$ & $\begin{array}{l}\text { radiative forcing (after stratospheric tempera- } \\
\text { ture adjustments) of } \mathrm{CF}_{4}\end{array}$ & 2 \\
\hline $\begin{array}{l}\text { Radiative Forc- } \\
\text { ing }\end{array}$ & $\begin{array}{l}\text { Radiative ForcinglAnthropogenic|F- } \\
\text { GasesISO2F2 }\end{array}$ & $\mathrm{Wm}^{-2}$ & $\begin{array}{l}\text { radiative forcing (after stratospheric tempera- } \\
\text { ture adjustments) of sulfuryl fluoride }\left(\mathrm{SO}_{2} \mathrm{~F}_{2}\right)\end{array}$ & 2 \\
\hline $\begin{array}{l}\text { Radiative Forc- } \\
\text { ing }\end{array}$ & $\begin{array}{l}\text { Radiative Forc- } \\
\text { ing|Anthropogenic|Montreal Gases }\end{array}$ & $\mathrm{Wm}^{-2}$ & $\begin{array}{l}\text { radiative forcing (after stratospheric tempera- } \\
\text { ture adjustments) of Montreal gases }\end{array}$ & 2 \\
\hline $\begin{array}{l}\text { Radiative Forc- } \\
\text { ing }\end{array}$ & $\begin{array}{l}\text { Radiative } \\
\text { ing|Anthropogenic|Montreal } \\
\text { Gases|CCl4 }\end{array}$ & $\mathrm{Wm}^{-2}$ & $\begin{array}{l}\text { radiative forcing (after stratospheric tempera- } \\
\text { ture adjustments) of } \mathrm{CCl}_{4}\end{array}$ & 2 \\
\hline
\end{tabular}


Table S4. Continued.

\begin{tabular}{|c|c|c|c|c|c|}
\hline Category & Variable & & Unit & Definition & Tier \\
\hline $\begin{array}{l}\text { Radiative Forc- } \\
\text { ing }\end{array}$ & $\begin{array}{l}\text { Radiative } \\
\text { inglAnthropogenic|Montreal } \\
\text { Gases|CFC|CFC113 }\end{array}$ & Forc- & $\mathrm{Wm}^{-2}$ & $\begin{array}{l}\text { radiative forcing (after stratospheric tempera- } \\
\text { ture adjustments) of CFC113 }\end{array}$ & 2 \\
\hline $\begin{array}{l}\text { Radiative Forc- } \\
\text { ing }\end{array}$ & $\begin{array}{l}\text { Radiative } \\
\text { ing|Anthropogenic|Montreal } \\
\text { Gases|CFCICFC114 }\end{array}$ & Forc- & $\mathrm{Wm}^{-2}$ & $\begin{array}{l}\text { radiative forcing (after stratospheric tempera- } \\
\text { ture adjustments) of CFC114 }\end{array}$ & 2 \\
\hline $\begin{array}{l}\text { Radiative Forc- } \\
\text { ing }\end{array}$ & $\begin{array}{l}\text { Radiative } \\
\text { ing|Anthropogenic|Montreal } \\
\text { Gases|CFC|CFC115 }\end{array}$ & Forc- & $\mathrm{Wm}^{-2}$ & $\begin{array}{l}\text { radiative forcing (after stratospheric tempera- } \\
\text { ture adjustments) of CFC115 }\end{array}$ & 2 \\
\hline $\begin{array}{l}\text { Radiative Forc- } \\
\text { ing }\end{array}$ & $\begin{array}{l}\text { Radiative } \\
\text { ing|Anthropogenic|Montreal } \\
\text { Gases } \mid \mathrm{CH} 2 \mathrm{Cl} 2\end{array}$ & Forc- & $\mathrm{Wm}^{-2}$ & $\begin{array}{l}\text { radiative forcing (after stratospheric tempera- } \\
\text { ture adjustments) of } \mathrm{CH}_{2} \mathrm{Cl}_{2}\end{array}$ & 2 \\
\hline $\begin{array}{l}\text { Radiative Forc- } \\
\text { ing }\end{array}$ & $\begin{array}{l}\text { Radiative } \\
\text { ing|Anthropogenic|Montreal } \\
\text { Gases|CH3Br }\end{array}$ & Forc- & $\mathrm{Wm}^{-2}$ & $\begin{array}{l}\text { radiative forcing (after stratospheric tempera- } \\
\text { ture adjustments) of } \mathrm{CH}_{3} \mathrm{Br}\end{array}$ & 2 \\
\hline $\begin{array}{l}\text { Radiative Forc- } \\
\text { ing }\end{array}$ & $\begin{array}{l}\text { Radiative } \\
\text { ing|Anthropogenic|Montreal } \\
\text { Gases } \mid \mathrm{CHCl} 3\end{array}$ & Forc- & $\mathrm{Wm}^{-2}$ & $\begin{array}{l}\text { radiative forcing (after stratospheric tempera- } \\
\text { ture adjustments) of } \mathrm{CHCl}_{3}\end{array}$ & 2 \\
\hline
\end{tabular}


Table S4. Continued.

\begin{tabular}{|c|c|c|c|c|c|}
\hline Category & Variable & & Unit & Definition & Tier \\
\hline $\begin{array}{l}\text { Radiative Forc- } \\
\text { ing }\end{array}$ & $\begin{array}{l}\text { Radiative } \\
\text { ing|Anthropogenic|Montreal } \\
\text { Gases|Halon1202 }\end{array}$ & Forc- & $\mathrm{Wm}^{-2}$ & $\begin{array}{l}\text { radiative forcing (after stratospheric tempera- } \\
\text { ture adjustments) of Halon-1202 }\end{array}$ & 2 \\
\hline $\begin{array}{l}\text { Radiative Forc- } \\
\text { ing }\end{array}$ & $\begin{array}{l}\text { Radiative } \\
\text { ing|Anthropogenic|Montreal } \\
\text { Gases|Halon1211 }\end{array}$ & Forc- & $\mathrm{Wm}^{-2}$ & $\begin{array}{l}\text { radiative forcing (after stratospheric tempera- } \\
\text { ture adjustments) of Halon- } 1211\end{array}$ & 2 \\
\hline $\begin{array}{l}\text { Radiative Forc- } \\
\text { ing }\end{array}$ & $\begin{array}{l}\text { Radiative } \\
\text { ing|Anthropogenic|Montreal } \\
\text { Gases|Halon1301 }\end{array}$ & Forc- & $\mathrm{Wm}^{-2}$ & $\begin{array}{l}\text { radiative forcing (after stratospheric tempera- } \\
\text { ture adjustments) of Halon-1301 }\end{array}$ & 2 \\
\hline $\begin{array}{l}\text { Radiative Forc- } \\
\text { ing }\end{array}$ & $\begin{array}{l}\text { Radiative } \\
\text { inglAnthropogenic|Montreal } \\
\text { Gases|HCFC141b }\end{array}$ & Forc- & $\mathrm{Wm}^{-2}$ & $\begin{array}{l}\text { radiative forcing (after stratospheric tempera- } \\
\text { ture adjustments) of } \mathrm{HCFC} 141 \mathrm{~b}\end{array}$ & 2 \\
\hline $\begin{array}{l}\text { Radiative Forc- } \\
\text { ing }\end{array}$ & $\begin{array}{l}\text { Radiative } \\
\text { inglAnthropogenic|Montreal } \\
\text { Gases|HCFC142b }\end{array}$ & Forc- & $\mathrm{Wm}^{-2}$ & $\begin{array}{l}\text { radiative forcing (after stratospheric tempera- } \\
\text { ture adjustments) of } \mathrm{HCFC} 22\end{array}$ & 2 \\
\hline $\begin{array}{l}\text { Radiative Forc- } \\
\text { ing }\end{array}$ & Radiative Forcing|Anthropoge & IOther & $\mathrm{Wm}^{-2}$ & $\begin{array}{l}\text { radiative forcing from factors not covered in } \\
\text { other categories (after stratospheric temperature } \\
\text { adjustments) }\end{array}$ & 2 \\
\hline
\end{tabular}


Table S4. Continued.

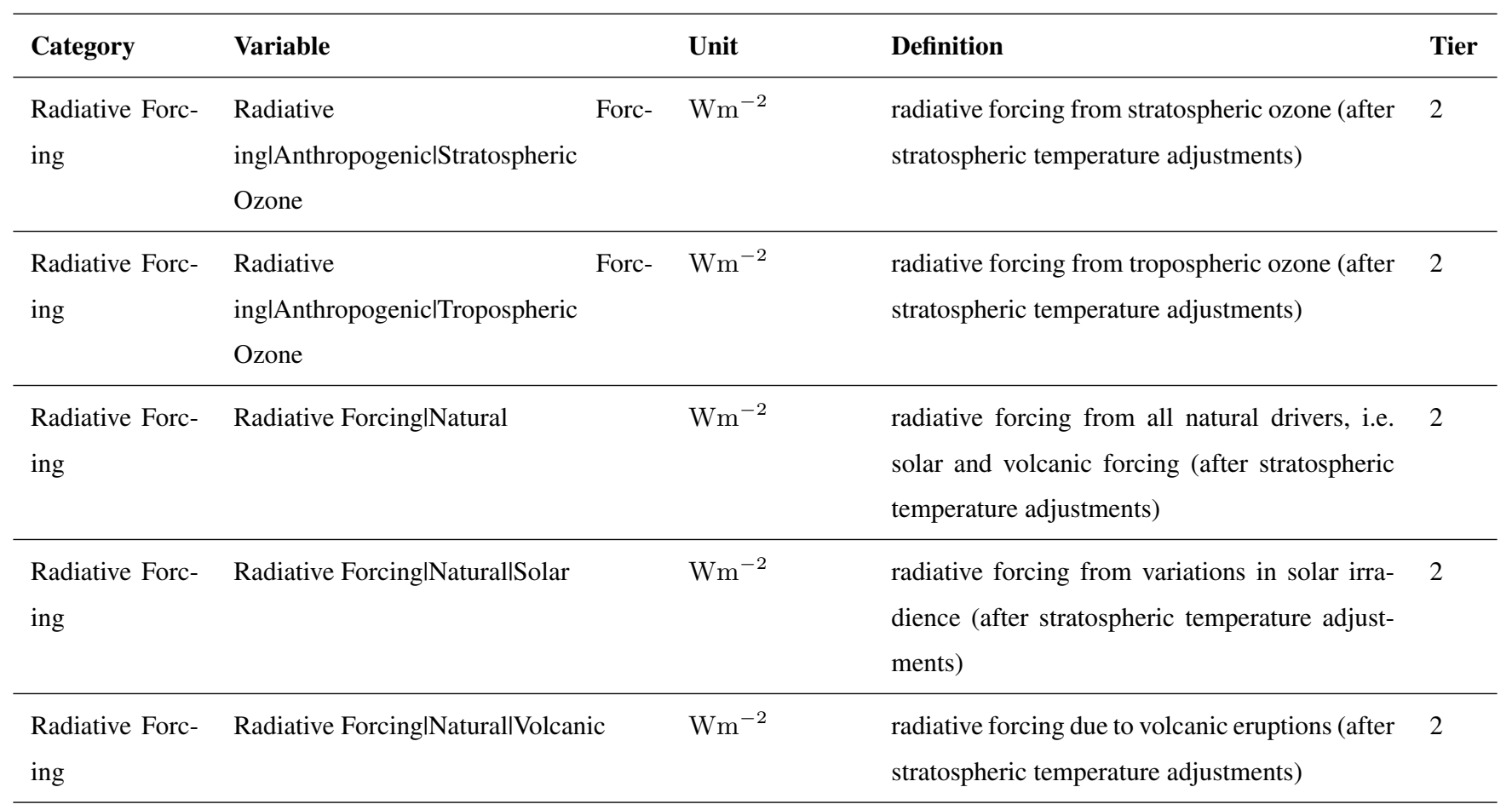




\section{References}

Gidden, M. J., Riahi, K., Smith, S. J., Fujimori, S., Luderer, G., Kriegler, E., van Vuuren, D. P., van den Berg, M., Feng, L., Klein, D., Calvin, K., Doelman, J. C., Frank, S., Fricko, O., Harmsen, M., Hasegawa, T., Havlik, P., Hilaire, J., Hoesly, R., Horing, J., Popp, A., Stehfest, E., and Takahashi, K.: Global emissions pathways under different socioeconomic scenarios for use in CMIP6: a dataset of harmonized emissions trajectories through the end of the century, Geoscientific Model Development, 12, 1443-1475, https://doi.org/10.5194/gmd-121443-2019, 2019. 